\title{
Project Summaries 1994
}

U.S. Department of Commerce

Technology Administration

National Institute of Standards and Technology 



\section{Project Summaries 1994}

NIST SP 838-5
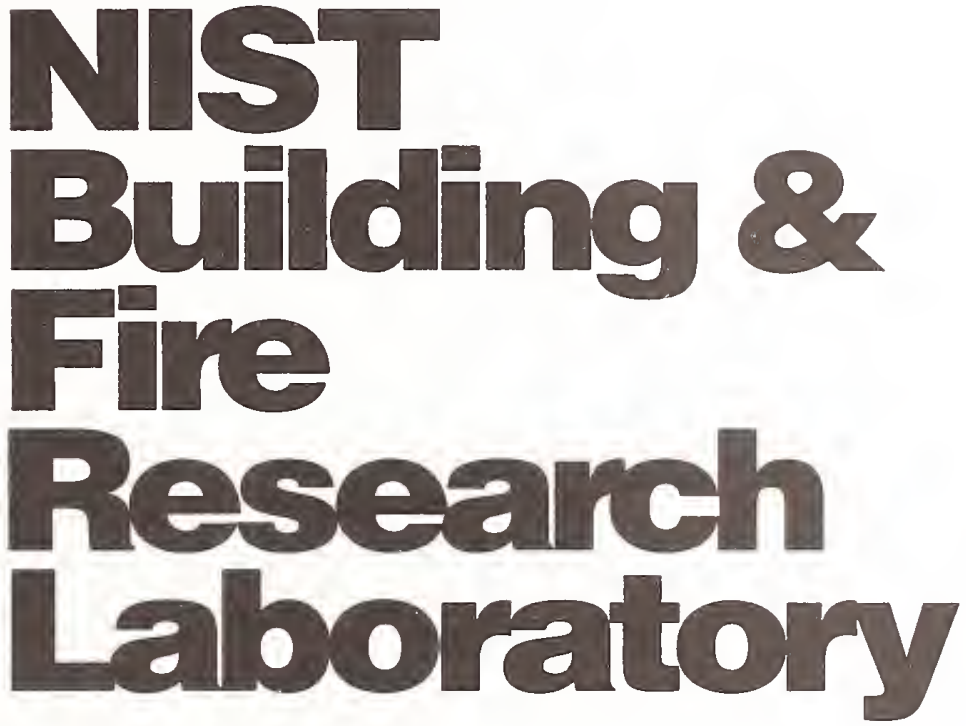

Noel J. Raufaste

June 1994

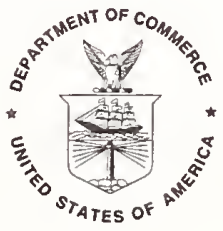

U.S. Department

of Commerce

Ronald H. Brown, Secretary

Technology

Administration

Mary L. Good,

Under Secretary for Technology

National Institute

of Standards and

Technology

Arati Prabhakar, Director 
National Institute of Standards and Technology Special Publication 838-5, Natl. Inst. Stand. Technol. Spec. Publ. 838-5 158 pages (June 1994) CODEN: NSPUE2
U.S. Goverment Printing Office Washington: 1994
For sale by the Superintendent of Documents U.S. Government Printing Office Washington, DC 20402 


\section{FOREWORD}

Construction is one of the Nation's largest industries. In 1993, new construction put in place amounted to about $\$ 470$ billion ( $7.9 \%$ of GDP) and provided about 6 million jobs. Fires and natural disasters destroy a significant portion of constructed facilities every year. Costs of fire safety and fire losses exceed $\$ 128$ billion a year. Natural disasters cause tens of billions of dollars annually. For example, during the 12 month period July 1993 through June 1994, the United States experienced significant property losses from natural disasters such as: the Mid-west Floods; the January 1994 Northridge Earthquake; and wind damage to the built environment. These are only three examples; many other natural phenomena occur each year. The quality of constructed facilities directly affects the productivity of the U.S. building and fire communities and affects the safety and quality of life of all constructed facilities. Over $60 \%$ of the nation's wealth is invested in constructed facilities.

The National Institute of Standards and Technology's (NIST) mission is to promote U.S. economic growth by working with industry to develop and apply technology, measurements, and standards. NIST's direct customer is U.S. industry. Its work yields direct investments with and benefits for the private sector -- in contrast to spinoffs derived from other federal investments.

NIST's Building and Fire Research Laboratory (BFRL), one of NIST's eight Laboratories, enhances the competitiveness of U.S. industry and public safety through performance prediction and measurement technologies and technical advances that improve the life cycle quality of constructed facilities.
BFRL's efforts are closely coordinated with complementary activities of industry, professional and trade organizations, academe, and other agencies of government. The vision for $B F R L$, the structure of its technical programs, and the determination and timing of its technical products are based on analyses of industry needs and BFRL's own unique resources and capabilities.

BFRL's laboratory facilities include: six-degree-of-freedom structural testing facility; large-scale structural testing facility with the $53 \mathrm{MN}$ (12-million pound) universal structural testing machine; environmental chambers; guarded hotplate; calibrated hot-box; plumbing tower; building materials imaging and modeling laboratory; large burn facility for conducting experimental fires in full-scale, large industrial fire test facilities, fire suppression test facilities; and a fire simulation laboratory.

$\mathrm{BFRL}$ is a major nonproprietary source of technical information for development of voluntary standards by organizations such as ASTM; American Concrete Institute; American Society of Heating, Refrigerating and Air-Conditioning Engineers; American Society of Civil Engineers; Institute of Electrical and Electronics Engineers; and the National Fire Protection Association. The resulting standards are widely used in building and fire codes.

BFRL works closely with its international peer organizations to maintain awareness of foreign research developments, as well as assure that generic research efforts are complementary, and U.S. interests are represented in the preparation of international standards and practices. BFRL cooperates closely with other U.S. and foreign laboratories in the conduct of its research. Eighty-three research 
associates from U.S. industry, guest researchers from foreign laboratories, and faculty members from universities worked at BFRL during 1993 for periods averaging about a year.

BFRL participates in over 100 national standardization activities; provides leadership in national and international standardization organizations and chairs more than 20 voluntary standardization activities. BFRL annually publishes over 230 reports, articles for research journals, and articles for professional and trade journals. BFRL staff annually makes hundreds of presentations to professional and technical meetings of building community organizations. For 24 years, BFRL has hosted a monthly Building Technology Symposia Series in cooperation with other organizations concerned with building research and practice and hosts weekly Fire Research Seminars for NIST staff and colleagues from the fire community. These are effective means of transferring the latest knowledge to practitioners and peer researchers. The Fire Research Information Service (FRIS) consisting of national and international fire research literature and FIREDOC, the automated database of fire research literature, is the only comprehensive national library resource for the fire community.

At the beginning of FY 1994, BFRL had about 200 employees of which 125 are professional staff, 65 have Ph.D.s, and 17 are registered engineers. BFRL's budget for FY 1994 is $\$ 26$ million. Funding comes from direct appropriations ( $\$ 14$ million), from other Federal agencies ( $\$ 12$ million) and from the private sector ( $\$ 2$ million).

This report summarizes BFRL's research for 1994 . The report is arranged by its research programs: structural engineering, materials engineering, mechanical and environmental systems, fire safety and engineering, and fire science. Each summary lists the project title, the BFRL point of contact, sponsor, research, and recent results.

For further information about BFRL, its facilities, opportunities for Guest Researcher assignments, collaborative programs, and contracted research, contact BFRL's Office of Cooperative Research Programs, Building 226, Room B250, NIST, Gaithersburg, MD 20899. Persons requesting information should contact BFRL using facsimile number 301-975-4032 or e-mail raufaste@micf.nist.gov. 
CONTENTS

iii Foreword

1 EARTHQUAKE ENGINEERING

2 Seismic Strengthening

Methodologies for Reinforced

Concrete $(\mathrm{RC})$ Frame Buildings

3 Seismic Performance of Precast Concrete Connections

5 Seismic Design of Non-Structural Components and Secondary Systems

6 Seismic Resistance of Joints in Precast Concrete Shear Walls

7 Seismic Resistance of PartiallyGrouted Masonry Shear Walls

9 Performance Requirements for Passive Energy Dissipation Systems for Buildings and Lifeline Structures

10 Establishment of Lifeline Seismic Safety Executive Board

11 Mitigating Liquefaction Potential

13 Management of the Interagency Committee on Seismic Safety in Construction (ICSSC)

14 Support Implementation of Executive Order 12699

14 Technical Assistance to FEMA Project Officer (Existing Buildings)

15 Secretariat U.S.-Side Panel on Wind and Seismic Effects

\section{STRUCTURAL BEHAVIOR}

17 Noise-Induced Chaos and Chaotic Transport

17 Automated Construction Site Metrology

18 High Performance Concrete

19 Effects of Tendon Duct Grease Leakage on the Integrity of Prestressed Concrete Containment

20 Next Generation Design Standard for Wind Loads

21 Design Wind Load Criteria for Manufactured Housing

22 Calibration of Marshall Compaction Hammers

23 Building and Fire Code Liaison

24 CONCRETE

25 Microstructure-Property

Relationships in Random Porous Materials

26 Transport Properties of HighPerformance Concrete

27 Integrated Knowledge System for High-Performance Concrete

27 Computation of the MicroMechanical Properties of CementBased Materials

28 Quantitative X-Ray Powder Diffraction Analysis of Clinker and Cement

29 Factors Affecting the Performance of Beneficiated Fly Ash in Cement 
Performance Criteria for Long

Lived Concrete for Radioactive

Waste Storage

31 Concrete Degradation Computer

Code for LLW Performance

Assessments

\section{COATINGS}

33 Service Life of Coating Systems

34 Organic Coatings

35 Standards for Abatement of

Hazards from Lead in Buildings

36 Method for Evaluation of

Performance of Lead-in-Paint

Measuring Devices Under

Simulated Field Conditions

38 Development of Method for

Leaching of Lead from Lead-Based

Paint Through Encapsulants

39 Performance Criteria for Selection of Liquid-Coatings Encapsulants for Lead-Based Paint

Long-Term Performance of

Polymer-Based Encapsulants for

Lead-Based Paint on Interior Walls

41 Technical Assistance on Lead-

Based Paint Issues

42 QUALITY ASSURANCE

43 Cement and Concrete Reference Laboratory

44 AASHTO Materials Reference Laboratory

45 Construction of Addition to CMRL Proficiency Sample Building
45 Durability of Polymer/Glass Fiber Composites for Building Applications

47 Performance Criteria for Single-Ply Roofing Membranes

48 Serviceability of Roofing Systems

49 Materials and Processes for Durable Relocatable Rigid Wall Structures

50 THERMAL MACHINERY

51 Thermodynamic Performance of Natural Fluids as Alternative Refrigerants

52 Thermodynamic Performance of Alternative Refrigerants and Refrigerant Mixtures

53 Calorimetric and Visual Study of Boiling Enhancements with Refrigerant 134a and Horizontal Two-Phase Flow of R-32/134a

55 Mixtures of Fluorocarbons as Alternatives for R11, R12, R22, and $\mathrm{R} 502$

56 Minimizing Throttling Losses in the Refrigeration Cycle

\section{MECHANICAL SYSTEMS AND} CONTROLS

58 Communication Protocols for Building Controls

59 Real Time Fault Detection and Diagnostics

60 Test Procedures for Furnaces, Boilers, and Integrated Appliances 
61 Test Procedures for Heat Pumps and Air Conditioners

62 Development of Revised Test Procedures for RefrigeratorFreezers

63 Test Procedures for Plumbing Products

64 Development of Revised Test Procedures for Pool and Spa Heaters

\section{INDOOR AIR QUALITY}

66 Development and Application of Multizone Indoor Air Quality Models

67 Development of Protocols for Ventilation System Performance Evaluation

68 Infiltration/Ventilation - Large Buildings

70 Radon Entry and Mitigation in Large Buildings

71 Study of IAQ Improvement Through the Use of HVAC Systems

72 Indoor Air Quality Commissioning Program for TWFN

73 Indoor PAH and Particulate Emissions from Wood Stoves

74 COMPUTER INTEGRATED CONSTRUCTION

75 Advanced Manufacturing Systems and Networking Testbed (AMSANT) for the Process Plant Industries
76 Process Plant Engineering and Construction: Structural Systems

77 STEP for the Building Industry

78 STEP for the Process Plant Industries

79 Computerized Maintenance Management Information

80 Prototype Requirements Management System

\section{HEAT TRANSFER}

82 Test Procedures for Advanced Thermal Insulation Products

83 Test Methods for Evaluating the Thermal Conductivity of Small Insulation Samples

84 Thermal Insulation SRM for Wall or Window Test Methods

84 Thermal Resistance Measurements on Foam Insulation and PowderPanel Insulation

85 Experimental Validation of NIST Moisture Transfer Model

86 Controlling Moisture in Walls/Roofs of Manufactured Housing

88 Moisture Control Guidelines for Attics, Cathedral Ceilings, Crawl Spaces, and Walls

LIGHTING TECHNOLOGY

91 Field Measurements and Modeling of Lighting Distributions

92 Lighting and HVAC Interactions 
93 Evaluation of Occupant Response to Lighting Retrofits

94 Test Procedures for Lighting Fixtures and Systems

\section{FIRE PROTECTION APPLICATIONS}

96 Large Scale Smoke Movement

97 Study of Heat and Smoke Movement and Their Influence on Detector and Sprinkler Response in Enclosed Spaces with Complex Ceiling Geometrics

99 Balanced Design

100 Risk Assessment/Performance Codes

100 Enhanced GSA Engineering Fire Assessment System

101 FPEtool Advancement

101 Modification of Fire Protection Requirements (Performance Based Fire Protection for Nuclear Power Plants)

102 Emergency Egress from Air Traffic Control Towers (ATCT)

102 Fire and Thermal Characteristics of Navy Firefighting Trainers

103 Fire Research Information Service

104 Fire Research Bulletin Board System

104 Technology Transfer

105 Fire Research Seminars

\section{FIRE MODELING}

107 Ceiling Fires

108 Fire Model Verification

109 Development of the Fire Data Management System (FDMS) Database System for Fire Related Data

110 HAZARD Development

111 Modeling of Detector Response

111 Furniture Flammability

112 Support of Fire Modeling on the ex USS SHADWELL

113 Computing \& Network Resources

114 LARGE FIRE RESEARCH

115 Simulation of Turbulent Combustion and Transport in Fires

116 Development of Large Eddy Enclosure Fire Model

117 Analysis of Large Industrial Fires

118 In-Situ Burning of Oil Spills

119 Smoke Plume Sampling

120 Smoke Plume Trajectory from in situ Burning of Crude Oil in Alaska

120 Office Building Fire Research Program

121 Large Fire Test Facility

122 Cone/LIFT/Toxicity Support

123 Development of a Sizing Standard for Firefighter Protective Clothing 


\section{SMOKE DYNAMICS RESEARCH}

125 Carbon Monoxide Production and Prediction

126 Study of Smoke Agglomerates

126 Species Production for Turbulent Flame Modelling

127 Short-Duration Autoignition Temperatures of Liquid Hydrocarbon Fuels

129 MATERIALS FIRE RESEARCH

130 Polymer Flammability Modeling

131 Burning Rate of Materials

132 Char Structure and Flammability

133 Flame Retardant Study on Engineering Plastics

134 Flammability of Composite Materials

135 Combustion of Silicone

136 Radiative Ignition and Subsequent Flame Spread Over Cellulosic Materials

137 Fire Resistant Aircraft Materials

138 FIRE SENSING AND EXTINGUISHMENT

139 Suppression of Simulated Engine Nacelle Fires

140 Risk Analysis for the Fire Safety of Airline Passengers

141 Advanced Fire Detection Systems
142 Protection of Data Processing Equipment with Water Sprays

143 Agent/System Compatibility for Halon 1301 Aviation Replacement

144 Agent Screening for Halon 1301 Aviation Replacement

145 Dynamics of Fire Suppression

146 Optimization of Aircraft Fire Suppression System Discharge

147 Real-Time Suppressant Concentration Measurement

148 Comparison of Fire Sprinkler Piping Materials, Steel, Copper, Chlorinated Polyvinyl Chloride, and Polybutylene, in Residential and Light Hazard Installations

149 Smoke Movement and Smoke Layer Development in High Bay Areas 

STRUCTURES

DIVISION

\section{EARTHQUAKE}

ENGINEERING 


\section{Seismic Strengthening Methodolo- gies for Reinforced Concrete (RC) Frame Buildings}

\author{
Principal Investigator: \\ Long Phan \\ Structures Division \\ 301-975-6077
}

\section{Diana Todd}

Structures Division

301-975-5296

\section{Sponsor:}

National Institute of Standards and Technology

\section{Objective}

To develop seismic strengthening guidelines for lateral load resisting capacity of lightly reinforced concrete $(\mathrm{RC})$ frame buildings.

\section{Problem}

Most RC structures in the eastern United States are not designed to resist large earthquakes. Thus, in the event of a strong motion earthquake in this region, many $R C$ buildings, which were designed primarily for gravity loads, are vulnerable to severe damage or even to total collapse. In order to minimize such vulnerability, these structures need to be strengthened with lateral load resisting elements which provide adequate stiffness and strength to resist lateral loads produced by moderate earthquakes and sufficient ductility to absorb the imparted energy of a strong earthquake without collapse.

\section{Approach}

In general, significant improvement of building seismic performance can be realized if lateral loads are diverted from columns to lateral load resisting elements. To achieve this, a number of techniques, such as infilling the openings in the frames with $R C$ walls, bracing the frames using steel sections, or increasing the size of the columns or the beamcolumn joints and adding reinforcement, have been proposed and tested in laboratories worldwide. These experiments provided valuable insight concerning seismic performance of strengthened, lightly RC frames. However, a systematic examination of all available test data to produce an analytical technique for quantitative assessment of seismic performance up to failure of existing and strengthened RC frame buildings has not been conducted. In this project, such an analytical method will be developed for 1. evaluating the seismic capacity of $\mathrm{RC}$ frame buildings and identifying existing deficiencies; and for 2. predicting seismic performance of the buildings after strengthening and providing quantitative assessment of the improvement in seismic performance. Ultimately, this project aims to develop methodologies for designing seismic upgrading schemes for RC frame structures using infill shear walls.

A two-Phase approach is being followed to achieve the stated objectives. In Phase 1 , hysteresis failure models (three-parameter models $\alpha, \beta$, and $\gamma$ ) are being developed for 1 . existing frames, 2 . frames strengthened by cast-in-place infilled walls or precast concrete panels, and 3. monolithic shear walls. This family of hysteresis models will be incorporated into the platform program IDARC to improve its capability to analyze strengthened buildings. Once incorporated and validated, these models will provide an analytical tool for selecting an appropriate strengthening technique for a particular RC frame building.

In Phase 2, empirical expressions for quick estimation of parameters critical for retrofit design, such as ultimate seismic capacity $\mathrm{P}_{\mathrm{u}^{\prime}}$ story drift ratio at ultimate load $d_{u} / h_{c^{\prime}}$ and ductility factor $\mu_{u}\left(d_{u} / d_{y}\right)$, for existing RC frames and strengthened frames will be developed based on the same set of experimental data used in developing the hysteresis models. The two approaches should reinforce each other. The hysteresis models will provide in-depth analysis of the strengthened structures including complete hysteresis performance of the structure at each stage of loading or deformation. The empirical formulas provide a simplified method for computing the critical parameters which are needed for evaluating the improved seismic performance of the strengthened structure. Either technique could be used to assess the efficacy of various strengthening schemes for a particular building. Comparing the results of the two techniques will provide an additional validation.

Two analytical methods have been developed and proposed for use in estimating seismic performance of existing and strengthened RC frames. These techniques include hysteresis failure models and empirical formulas for $P_{u^{\prime}}, d_{u} / h_{c^{\prime}}$ and $\mu_{u}$ for four cases, including 1 . existing frames, 2 . existing monolithic frame-wall constructions, 3. frames strengthened by infilling the opening with CIP concrete walls, and 4 . frames strengthened by infilling the opening with precast concrete panels.

Research planned for FY 1994 includes validation of the hysteresis models and empirical formulas for use with multi-story, multi-bay structures; further refinement of empirical formulas, including formulas for R factors; parametric study using the developed models and formulas to confirm (or invalidate) experimental observations with aim to develop detail design guidelines for infilled frames. 
The results of this project are expected to produce guidelines for providing lateral load resisting elements. They will be used to evaluate the capacity of existing buildings and identify deficiencies; and to predict seismic performance of the buildings after strengthening and provide quantitative assessment of the improvement in seismic performance.

\section{Recent Results}

Phan, L.T., Todd, D.R., and Lew, H.S., Recommended Guidelines for Seismic Strengthening of RC Frames by the Infilling Technique, NISTIR, November 1993.

Phan, L.T., Todd, D.R., and Lew, H.S., "Empirical Expressions for Estimating Seismic Performance of Existing and Strengthened RC Frames," Abstract submitted to the 1993 SEAOC Annual Convention; September 29 to October 2, 1993; Scottsdale, Arizona.

Phan, L.T., Todd, D.R., and Lew, H.S., Empirical Expressions for Estimating Seismic Performance of Existing and Strengthened RC Frames, WERB reviewed and approved 9/22/1993.

Phan, L.T., Todd, D.R., and Lew, H.S., "Seismic Strengthening of Reinforced Concrete Frame Buildings," Proceeding of 1993 National Earthquake Conference, May 1993; Memphis, Tennessee.

Phan, L.T., Todd, D.R., and Lew, H.S., Strengthening Methodology for Lightly Reinforced Concrete Frames-I, NISTIR 5128, National Institute of Standards and Technology, Gaithersburg, MD 20899, February, 1993.
Seismic Performance of Precast Concrete Connections

\author{
Principal Investigator: \\ William Stone \\ Structures Division \\ 301-975-6075
}

Geraldine S. Cheok

Structures Division

301-975-6074

\section{Sponsor:}

National Institute of Standards and Technology

American Concrete Institute

Concrete Research and Education Foundation (ACI)

\section{Objective}

To develop technical data and recommend rational and consistent seismic design provisions for moment resistant precast concrete beam-column connections.

\section{Problem}

Careful attention to connection detail is required to ensure ductility and to prevent premature yielding of connections. In order to exploit the benefits that exist in precast construction, ATC-8, the Proceedings of the Workshop on Design of Prefabricated Concrete Buildings for Earthquake Loads, identified research on moment resistant joints between precast beam and column element as the top priority item.

Strength and ductility of joints of precast beamcolumn connections can be achieved by posttensioning the precast elements, combined with the use of special reinforcing details, and fiber reinforced grout in the joints. Technical data are needed to establish provisions for code and standards, thereby promoting precast construction in seismically active regions.

Currently, there is limited guidance regarding the design and detailing of precast concrete structures for seismically active regions. The 1985 Uniform Building Code (UBC) permits the use of precast concrete elements to resist seismic forces provided the design and detailing used satisfy the Code requirements for cast-in-place concrete structures. The 1985 edition of the UBC Code was in use at the time this program was initiated. It has been presumed that precast structures tend to be less ductile and tend to have a less stable inelastic response than do cast-in-place monolithic structures. This is primarily because the inelastic strains 
are concentrated in the connections. Thus, the connections are often unavoidable weak links.

\section{Approach}

The American Concrete Institute (ACI) has formed a task group of experts from the academic research community, the Precast/Prestressed Concrete Institute, the Portland Cement Association, and the design profession to provide technical guidance on specific parts of this project, for example, guidance in the design of specific joint details.

During FY 1994, BFRL will perform an experimental model study to characterize joint behavior. The experimental program is divided into four tasks.

1. Determine a viable connection.

2. Test several test specimens as determined in Task 1.

3. Examine the use of partially bonded tendons. 4. Examine the use of low strength steel used in conjunction with the post-tensioning in a precast connection.

This Task is partially funded by Pankow Builders through the American Concrete Institute (ACI).

The results from the experimental tests will be used to determine the parameters required for either a "smooth" or multi-linear hysterectic failure model. The results from this test program will be used to develop design parameters such as $P w$ values used in the UBC code and will provide the design community a rational means of designing moment resisting precast frames.

Recent Results

Cheok, G.S. and Stone, W.C., Overview of NIST

Research on Seismic Performance of Moment

Resisting Precast Beam-Column Joints Containing

Post-Tensioning, NISTIR 5257, National Institute of

Standards and Technology, Gaithersburg, MD,

August 1993.

Cheok, G.S. and Stone, W.C., Performance of 1/3Scale Model Precast Concrete Beam-Column Connections Subjected to Cyclic Inelastic Loading Report No. 3, NISTIR 5246, National Institute of Standards and Technology, Gaithersburg, MD, August 1993.

Cheok, G.S. and Lew, H.S., "Model Precast Concrete Beam-to-Column Connections Subject to Cyclic Loading," PCl Journal, Vol. 38, No. 4, pp 8092, Chicago, IL, July-August 1993.

Cheok, G.S., Stone, W.C., and Lew, H.S., "Seismic Performance Behavior of Precast Concrete BeamColumn Joints," Structural Engineering in Natural
Hazards Mitigation, Vol. 1, Proceedings of the Structures Congress '93, American Society of Civil Engineers, pp 82-88, April 1993.

Cheok, G.S., Stone, W.C., and Lew, H.S., "Partially Prestressed and Debonded Precast Concrete BeamColumn Joints," Third Meeting of the U.S.-Japan Joint Technical Coordinating Committee on Precast Seismic Structural Systems, U. C. San Diego, November 18-20, 1992. 


\section{Seismic Design of Non-Structural Components and Secondary Systems}

\author{
Principal Investigator: \\ Andrew W. Taylor \\ Structures Division \\ 301-975-6078
}

\section{Sponsor:}

National Institute of Standards and Technology

\section{Objective}

To develop seismic design criteria for non-structural components and secondary systems.

\section{Problem}

Damage from earthquakes to non-structural components and secondary systems can have a greater effect on the safety of occupants and loss of property than the effects of structural damage. When buildings in a community are rendered nonfunctional due to non-structural damage or to inoperative secondary systems, the well-being of the community can be severely jeopardized. Furthermore, in economic terms, indirect losses (damaged equipment, lost inventory, lost records, and lost revenuel can be two to three times greater than the cost of replacing collapsed buildings or structures, as demonstrated by the aftermath of the 1989 Loma Prieta Earthquake.

Non-structural components and secondary systems include cladding, ceilings, building contents, equipment, and HVAC, plumbing, and electrical systems. Most the past National Earthquake Hazard Reduction Program (NEHRP) efforts have focused on improvements of the structural design of buildings to prevent total collapse. However, little attention has been paid to the performance of non-structural components and secondary systems.

Design guidelines need to be developed to ensure adequate seismic resistance of these systems, particularly with respect to the reduction of falling debris and the adequate anchorage of equipment in buildings and critical facilities such as schools, hospitals, fire stations, and emergency command centers. In studies conducted by the Nuclear Regulatory Commission, the performance of anchors has been identified as one of the most important factors for assuring the functionality of nuclear power plants during and after earthquakes.

\section{Approach}

This project was initiated in FY 1993. A broad and comprehensive review of the critical elements of the current state of practice, through literature searches and interviews with experts in this field was performed. It serves as the basis for FY 1994 to FY 1997 research activities.

During FY 1994, BF́RL will identify specific knowledge gaps related to the seismic performance of nonstructural elements and will initiate research to help fill in these gaps. Examples of topics to be investigated might include methods for restraining equipment, methods for determining potential damage to loose building contents, the design of seismic-resistant cladding attachment systems, or the design of seismic resistant pipe support systems.

In the last year of the study, FY 1997, draft seismic design guidelines will be developed for non-structural components and secondary systems in buildings. These guidelines will be written in practical terms so that they can be readily applied by engineers and building officials.

The results of this research are expected to improve earthquake safety and reduce economic losses from earthquakes through the developments of guidelines and standards for the seismic performance of nonstructural components and secondary systems.

Recent Results

A state-of-the-art report is in progress. 


\section{Seismic Resistance of Joints in Precast Concrete Shear Walls}

\author{
Principal Investigator: \\ Arturo E. Schultz \\ Structures Division \\ 301-975-5301
}

\section{Sponsor: \\ University of Nebraska-Lincoln \\ Department of Civil Engineering}

\section{Objective}

To determine the seismic performance of connections between precast shear wall panels, including strength, stiffness and energy dissipation characteristics and to develop modelling techniques and design guidelines for joint connection details in precast shear walls.

\section{Problem \\ Worldwide experience in previous earthquakes, including the 1964 Alaska, the 1976 Friuli (Italy) and the 1988 Armenia events, have demonstrated in a dramatic manner the weakness of precast construction when subjected to seismic motions. The problem stems from the current lack of knowl- edge regarding behavior of jointed construction, and the inability to define adequate design requirements for connections between precast elements. Due to this lack of information, U.S. building codes do not address the design of precast concrete specifically, and, as a consequence, loadbearing precast con- crete systems are seldom used in seismically active regions of the United States.}

\section{The PRESSS (Precast $\underline{\text { Seismic }} \underline{\text { Stuuctural }} \underline{\text { Systems) }}$} program was initiated by the National Science Foundation (NSF) in 1990, as part of the National Earthquake Hazard Reduction Program (NEHRP), to develop new structural forms, to advance modelling techniques and to propose design procedures for seismic design of precast concrete buildings in the United States. As part of PRESSS Phase II, the NSF funded a multi-institution research program at the University of Nebraska-Lincoln (UNL) to study the seismic resistance of a six-story totally precast office building exposed to moderate seismicity. The building features separate vertical and lateral load resisting systems, with shear walls and architectural perimeter frames comprising the latter. This project is a task of the UNL program; it seeks to study the cyclic lateral load behavior of horizontal and vertical joints between precast panels in shear walls for improving seismic design.

\section{Approach}

During FY 1994, BFRL will perform cyclic lateral load tests of a maximum of 12 precast wall panel specimens during the 2 year duration of the project (the scope of the experimental work will depend on the funding level). The principal thrust of the research program is development of physical data for a wide variety of connections; the variables are connection detail and type of joint. Each of the first six wall panel specimens will feature a different vertical joint connection detail, and the remaining six specimens will feature a different horizontal joint connection detail. The vertical joint specimens comprise the localized region immediately adjacent to the vertical joint connection, while horizontal joint specimens include the entire panel above the joint and a stub representing the panel below. The horizontal joint tests feature uniformly-distributed, constant, vertical compression stresses on the joint, while the vertical joints will be tested in the absence of vertical stress. Tests for each horizontal joint specimen include an elastic phase which is intended to simulate the performance of top-story panels, and an inelastic phase to simulate the behavior of first-story panels. The ratio of story shear to overturning moment will be different in these two types of tests to reflect the loading conditions prevalent for top-story and first-story panels. For the vertical joint specimens, the loading configuration is the same in both phases, only the magnitude of the force/displacement demand differs.

BFRL will test the three vertical joint specimens that were designed, fabricated and cast in FY 1993. The other vertical joint specimens and the horizontal joint specimens, all which were designed and detailed in FY 1993, will be cast and tested in FY 1994.

The experimental data from this program will verify proposed design procedures for precast concrete panel connections and to calibrate current analytical models for precast concrete structural systems. Superior analysis and design models for precast concrete shear walls can provide a tremendous boost to the precast concrete construction industry both in the United States and abroad.

\section{Recent Results}

Schultz, A.E., Magana, R.A., Tadros, M.K., and Huo, X., "Experimental Study of Joint Connections in Precast Concrete Walls," Proceedings, Fifth U.S. National Conference on Earthquake Engineering, Chicago, IL, July 10-14, 1994 (paper accepted).

Schuitz, A.E., Tadros, M.K., Huo, X.-M., and Magaña, R.A., Seismic Resistance of Vertical Joints in Precast Shear Walls, Proceedings, XII FIP Congress, Washington, DC, May 30-June 2, 1994. 
Tadros, M.K., Einea, A., Low, S.-G., Magaña, R.A., and Schultz, A.E., Seismic Behavior of a Six-Story Precast Office Building, Proceedings, XII FIP Congress, Washington, DC, May 30-June 2, 1994.

Tadros, M.K., Einea, A., Low, S.-G., Magaña, R.A., and Schultz, A.E., Seismic Resistance of a Six-Story Totally Precast Office Building, Proceedings, FIP Symposium '93, Kyoto, Japan, October 17-20, 1993.

Schultz, A.E., Tentative Connection Details for Horizontal Joints Berween Precast Wall Panels in Earthquake-Resistant Precast Concrete Buildings, Department of Civil Engineering, North Carolina State University, Raleigh, NC, 26 pp, November 1992.

Tadros, M.K., Schultz, A.E., and Magaña, R.A., PRESSS: Progress Through Year Two, "R \& D Leading the Way to Quality Improvement," Session 2, $1992 \mathrm{PCl}$ Convention, Nashville, TN, October 12, 1992.

Schultz, A.E., Seismic Resistance of Horizontal Connections for Precast Concrete Panels, Department of Civil Engineering, North Carolina State University, 20 pp, Raleigh, NC, July 1992.
Seismic Resistance of Partially Grouted Masonry Shear Walls

Principal Investigator:

Arturo Schultz

Structures Division

301-975-5301

\section{Sponsor:}

National Institute of Standards and Technology

\section{Objective}

To develop seismic resistant design guidelines of partially grouted and lightly reinforced masonry shear walls for their use in regions of moderate seismicity.

\section{Problem}

The behavior of masonry shear walls has been the subject of increased attention during the past 15 years. However, this attention has addressed primarily those fully grouted, reinforced walls. Due, in part, to the limited amount of physical data on the response of partially grouted masonry walls to in-plane lateral loads, no acceptable design formulas are available for predicting shear strength of such walls. The limited data available also suggests that empirical formulas for shear strength of fully grouted, reinforced walls have very limited applicability for determination of the shear strength of partially grouted, lightly reinforced shear walls.

Recent research activities on the seismic resistance of masonry walls at BFRL include a literature survey that identified approximately 700 tests of masonry walls and panels subjected to in-plane shear. Many of these tests used diagonal compression panels which do not simulate shear wall behavior, and only a few featured partially grouted masonry. Subsequent masonry research at BFRL has included:

1. Modification and calibration of an existing empirical formula for predicting shear strength of fully grouted, reinforced masonry shear walls.

2. Testing of wall specimens, which contain no vertical reinforcement, to investigate the influence of longitudinal reinforcement ratio on in-plane shear strength.

3. Predicting shear strength of fully grouted, reinforced masonry shear walls using the finite element method.

The current module of the ongoing, multi-year BFRL research plan on masonry walls seeks to define the cracking and ultimate strengths of partially grouted, lightly reinforced masonry shear walls, as well as the associated deformations. This module was initiated in FY 1993 in which 1. the research 
program on partially grouted masonry shear walls was developed, 2. the hardware needed for constructing, transporting, and testing the specimens was designed and fabricated, and 3. the first series of partially grouted shear wall specimens was designed. The data to be generated from these tests is an essential part of this program, and it is needed to formulate the analytical methods for prediction of strength and deformation.

\section{Approach}

The first 1 year phase seeks to study replacement of horizontal reinforcement with bed joint reinforcement; the second and third 1 year phases will evaluate the influence of horizontal reinforcement, axial stress and aspect ratio on partially-grouted masonry shear wall behavior. The third phase also includes an effort to develop semi-empirical, simplified design expressions for the purpose of predicting shear strengths and deformations of partiallygrouted masonry shear walls.

During FY 1994, BFRL will test the first series of specimens of highest priority, which target the effects of type and amount of horizontal reinforcement, and height-to-length aspect ratio. This series of experiments will be conducted on six specimens of partially-grouted hollow masonry shear walls. The objective is to study the influence of the type of horizontal reinforcement and aspect ratio on shear behavior of walls in which all horizontal reinforcement is provided as bed joint reinforcement instead of the more traditional practice of placing deformed bars in grouted bond beams.

Analytical activities will be conducted in parallel with the experiments, and these include development of semi-empirical simplified expressions for predicting cracking and ultimate shear strengths and deformations. Similar efforts at BFRL led to the improvement of a shear strength formula for fullygrouted, reinforced masonry shear walls. There also is a pressing need to study the behavior of partiallygrouted masonry shear walls using a more systematic procedure that can give global as well as local information regarding states of stress, strain, cracking and yielding. For this purpose, the ongoing effort in finite element analysis will be expanded to the case of partially grouted walls. This effort will serve to calibrate existing finite element models to establish a more detailed understanding of the behavior of partially grouted, lightly reinforced masonry shear walls by formulating new models.

The results of this research are expected to have a high potential for improving the productivity and enhancing the cost-effectiveness of the U.S.

masonry construction industry.
Recent Results

Schultz, A.E., "Performance of Masonry Structures under Extreme Lateral Loading Events," Chapter 6 in Masonry in the Americas, D.P. Abrams ed., ACl Special Publication, American Concrete Institute, Detroit, MI, December 1993 (manuscript accepted for publication).

Fattal, S.G., The Effect of Critical Parameters on the Behavior of Partially-Grouted Masonry Shear Walls Under Lateral Loads, NISTIR 5116, National Institute of Standards and Technology, Gaithersburg, MD, June 1993.

Fattal, S.G., Research Plan for Masonry Shear Walls, NISTIR 5117, National Institute of Standards and Technology, Gaithersburg, MD, June 1993.

Fattal, S.G., Strength of Partially-Grouted Masonry Shear Walls Under Lateral Loads, NISTIR 5147, National Institute of Standards and Technology, Gaithersburg, MD, June 1993. 
Performance Requirements for Passive Energy Dissipation Systems for Buildings and Lifeline Structures

\author{
Principal Investigator: \\ Harry Shenton \\ Structures Division \\ 301-975-6067
}

\section{Sponsor:}

National Institute of Standards and Technology

\section{Objective}

To develop guidelines for pre-qualification, prototype and quality control testing of seismic isolation systems.

\section{Problem}

Seismic isolation has been proven to be effective in reducing the level of response in structures during strong earthquake ground shaking. In practice, the components of the isolation system undergo an extensive series of prototype and quality control tests before installation: BFRL recently completed draft guidelines for conducting these tests. The draft guidelines were developed with the assistance of a five member Oversight Committee of experts from the field of isolation.

To foster support and wider adoption of the proposed guidelines, the isolation community at large must be given the opportunity to review, comment and propose changes to the guidelines. Furthermore, the guidelines must be assessed and evaluated by conducting tests on typical isolation components, to expose inconsistencies, omissions of important data and other unforeseen problems with the procedures. The industry review and experimental program will provide feedback for drafting the final "Guidelines for Testing...," to be completed in FY 1995.

\section{Approach}

During FY 1994, BFRL will:

1. Complete Draft Guidelines. Final review comments and recommendations from the Oversight Committee have been incorporated into the draft guidelines. The draft guidelines have been published as a set of three NIST internal reports (see Recent Results below).

2. Industry Review. Draft reports will be distributed to key individuals in industry for review and comment. This will include subcommittees of SEAOC, AASHTO and $\mathrm{ACl}$; vendors; manufacturers; structural engineers; bridge engineers; etc.
3. Review Workshop. A one-day workshop will be convened to provide a forum for review and discussion of the draft guidelines. Twenty to thirty experts from industry will be invited to participate. Comments and suggested changes will be discussed among participants. Results of the workshop will be summarized in minutes or notes. 4. Test Program for Assessing and Evaluating Guidelines Planning. A detailed plan will be developed for the experimental program. The plan will include a schedule of tests, man power, equipment and material needs that will be provided to the laboratory coordinator and technical staff. Tests will be initiated in FY 1994, pending funding availability and continue into FY 1995.

5. Summarize feedback. BFRL will compile and summarize feedback from the industry review, workshop and test program and provide feedback to the Oversight Committee.

6. Report of Results. Depending on the schedule of the various tasks, results will be summarized and reported in one or more NIST internal reports. Results will be submitted for publication in an appropriate technical journal.

The results of this research will produce a draft standard serving as a resource document for voluntary standard/specification writing bodies.

\section{Recent Results}

Shenton III, H.W., Draft Guidelines for Pre-Qualification and Prototype Testing of Seismic /solation System, NISTIR 5359, National Institute of Standards and Technology, Gaithersburg, MD, March 1994.

Shenton III, H.W., Draft Guidelines for Quality Control Testing of Sliding Seismic Isolation System, NISTIR 5371, National Institute of Standards and Technology, Gaithersburg, MD, March 1994.

Shenton III, H.W., Draft Guidelines for Quality Control Testing of Elastomeric Seismic Isolation System, NISTIR 5345, National Institute of Standards and Technology, February 1994.

Shenton III, H.W. and Lin, A.N., "Relative Performance of Fixed-Base and Base-Isolated Concrete Frames," ASCE Journal of the Structural Division, Vol. 119, No. 10, October 1993.

Shenton III, H.W., "Draft Guidelines for Testing and Evaluation of Seismic Isolation Systems," ATC17-1, Proceedings of a Seminar on Base /solation, Passive Energy Dissipation and Active Control, San Francisco, California, March 11-12, 1993.

Shenton III, H.W., "Design Criteria for Base Isolation of Buildings," Wind and Seismic Effects, Proceed- 
ings of the 24th US-Japan Panel on Wind and Seismic Effects, NIST SP843, National Institute of Standards and Technology, Gaithersburg, MD, September 1992.

Shenton III, H.W., Lin, A.N., and Lew, H.S., "Performance of Fixed and Isolated Structures," Tenth World Conference on Earthquake Engineering, Vol. 7, pp 2485-2490, Madrid, Spain, July 1992.

Lin, A.N., "Design Force Levels for Base-Isolated Structures," Proceedings 1992 ClB World Congress, Montreal, Canada, May 19, 1992.

Lin, A.N., and Shenton III, H.W., "Seismic Performance of Fixed-Base and Base-Isolated Steel Frames," ASCE Journal of Engineering Mechanics, Vol. 118, No. 5, May 1992.

\section{Establishment of Lifeline Seismic Safety Executive Board}

Principal Investigator:

Riley M. Chung

Structures Division

301-975-6062

\section{Sponsor:}

National Institute of Standards and Technology

Objective

To establish a Lifeline Seismic Safety Executive Board, LSSEB with the mission to implement the National Plan for developing and adopting seismic design guidelines and standards for lifelines.

\section{Problem}

Lifeline systems includes electrical power, gas and liquid fuels, telecommunications, transportation, and water supply and sewers. These public works and utilities systems that support human activities are vulnerable to earthquakes. The January 17, 1994 Northridge Earthquake in California is a good example of the impact of lifeline disruption on our built environment. A significant portion of the losses from the earthquake are related to lifelines.

Public Law 101-614 mandated that NIST, working with FEMA create a Plan for developing and adopting seismic design and construction standards for lifelines. NIST's BFRL has worked with private sector experts and developed a draft Plan, which was reviewed by the National Earthquake Hazard Reduction Program (NEHRP) Advisory Committee in 1992. The revised draft Plan was submitted to OMB through the Federal Emergency Management Agency (FEMA) in the spring of 1993. In September 1993, FEMA incorporated OMB comments and is now ready for submittal to the Congress for funding to support the Plan's execution.

\section{Approach}

The Plan asks Congress to support this effort beginning in FY 1995 and its first task is to establish a Lifeline Seismic Safety Executive Board (LSSEB). LSSEB is made of three components: its Board of Directors, the Lifeline Directorates, and the Executive Directorate.

The Board of Directors will consist of a chair and 12 to 15 technical experts recommended by major lifeline organizations, with balance between researchers and practitioners. The Board of Directors will define and address the needs and issues of all five lifeline systems to develop a prioritized overall program plan. The activities commissioned by the Board of Directors will be carried out by one of the 
Lifeline Directorates. A separate Directorate will be established for each of the five lifeline systems. The Directorates will address the specific issues and concerns in each lifeline category as well as conduct specific technical studies of relevance to the various lifelines. Each Directorate may have six to ten experts, selected by the Board of Directors. The Executive Directorate will include a full-time director and staff to provide management and administrative support to the Board of Director and Lifeline Directorates.

Subsequent to the Plan's activation, BFRL and FEMA will convene an ad hoc working group of six to eight individuals to nominate and select the chair and members of the Board of Directors and the director and staff of the Executive Directorate. Upon completion of these tasks, the working group will be dissolved.

Establishment of LSSEB will set in place the central organizations to execute the national Lifeline plan based on the priority developed by the Lifeline community. It will lead to early fruition, the development of design guidelines for various types of Lifeline systems. Implementation of these guidelines will result in significant economic loss reduction of lifelines from future earthquakes.

\section{Recent Results}

New project.
Mitigating Liquefaction Potential

Principal Investigator:

Riley Chung

Structures Division

301-975-6062

\section{Sponsor:}

National Institute of Standards and Technology

Objective

To develop guidelines for ground improvement for assessing the effectiveness of ground improvement and liquefaction potential using in-situ shear-wave velocity relations and system identification estimates of stiffness.

\section{Problem}

Lifeline systems are vulnerable to large earthquake induced ground displacements, such as those associated with liquefaction, land slides, slope failures and fault displacements. In many instances it is feasible to locate critical structures and support facilities on sites that are not susceptible to large ground displacements. However, such precautions are not always possible for long linear system elements such as pipelines, communication and electrical transmission lines, highways, and rail lines. Furthermore, large portions of the Nation's existing infrastructure are vulnerable because of location or age. Even when replacing aging systems, it is not always possible to relocate these systems to less vulnerable areas.

In the case of existing or unrelocatable systems, ground remediation is the only solution. Known ground improvement methods include drainage, densification, and soil modification by grouting. Important problems to be addressed include cost effectiveness, quantification of the soil condition, and in-situ determination of the degree of success obtained by ground treatment. The greatest impediment to ground improvement is cost.

\section{Approach \\ During FY 1994, BFRL will determine and alter the liquefaction susceptibility of a given soil profile. The approach involves development of design guidelines for soil improvement and development of methods to determine pre- and post-improvement against liquefaction propensity.}

The design provisions for the remediation of liquefiable soils will be based on the current state-of-theart of ground improvement including cost consideration. Determination of the state-of-the art will involve examination of the literature, consultation with and evaluation by leading practitioners and academic experts through a workshop. A program 
of needed future research topics will follow from the drafting of the design guidelines.

There exists a good correlation between shear-wave velocity and accepted methods of estimating liquefaction potential. Bechtel Corporation, San Francisco, will make available several sites and data, services, and equipment. The knowledge will allow derivation of a rational correlation between shearwave velocity and liquefaction potential.

Current work on the use of system identification techniques to estimate soil properties from earthquake strong motion will be extended to a wide range of strains to allow accurate calculation of insitu stiffness and damping. BFRL also will collaborate with the Electric Power Research Institute (EPRI) who will provide data from the Lontung, Taiwan, test site.

Development of sound design guidelines and other practices will lead to better lifeline systems to resist strong ground shaking from future earthquakes, resulting in significant reduction of economic losses.

\section{Recent Results}

Glaser, S.D., "Application of Parametric Models for Estimation of Soil Parameters," 8th International Conference of the Association for Computer Methods and Advances in Geomechanics, 1994, accepted for publication.

Glaser, S.D., "Characterizing the Behavior of Liquefying Sand by the Use of System Identification," ASCE Journal of Geotechnical Engineering, 1994, in preparation.

Glaser, S.D., Estimating In-Situ Liquefaction Potential and Permanent Ground Displacements Due to Liquefaction for the Siting of Lifelines, NISTIR 5150, National Institute of Standards and Technology, Gaithersburg, MD, 1993.

Glaser, S.D., Estimating Soil Parameters important for Lifeline Siting Using System Identification Techniques, NISTIR 5143, National Institute of Standards and Technology, Gaithersburg, MD, 1993.

Glaser, S.D., "Estimation of Liquefaction Potential by In-Situ Methods: State-of-the-Art," ASTM Journal of Geotechnical Testing, 1993, (submitted for publication).

Glaser, S.D., "Estimation of Surface Displacements due to Earthquake Excitation of Saturated Sands," EER/ Spectra, 1993, (submitted for publication).

Glaser, S.D., "A Review of the Use of System Identification to Characterize Earthquake-Excited
Soil," ASCE Journal of Geotechnical Engineering, 1993, (submitted for publication). 
Management of the Interagency Committee on Seismic Safety in Construction (ICSSC)

Principal Investigator:

Diana Todd

Structures Division

301-975-5296

Sponsor:

Federal Emergency Management Agency

Mitigation Directorate

\section{Objective}

To facilitate and expedite the work of the Interagency Committee on Seismic Safety in Construction (ICSSC) by providing the Chair and Secretariat and to provide technical assistance in development of draft standards for assessing and enhancing the seismic safety of existing Federal buildings suitable for adoption by the President by December 1, 1994.

\section{Problem}

The National Earthquake Hazard Reduction Program (NEHRP) established the ICSSC to assist Federal departments and agencies develop, improve, and maintain seismic safety programs for all types of construction. Thirty Federal departments and agencies participate in the ICSSC. The National Earthquake Hazard Reduction Act lamended by Public Law 101-614) designates the director of NIST to serve as the chair of the ICSSC. NIST and BFRL provides the Secretariat to the ICSSC, to facilitate, document, and disseminate the work of the Committee. The ICSSC is currently developing seismic evaluation and rehabilitation standards for existing Federally owned and leased buildings.

\section{Approach}

During FY 1994, BFRL will schedule, chair, and document at least two meetings per year of the full committee and at least one meeting per year of the steering committee. BFRL will produce agendas for and minutes of these meetings, will maintain membership rosters, and will perform other administrative tasks as needed to maintain the smooth functioning of this committee.

Key projects that the ICSSC will conduct in FY 1994 will include development of guidelines for implementing a proposed Executive Order on seismic safety of existing Federal buildings and updating their guidance for implementing Executive Order 12699 on seismic safety of new Federal buildings.
Todd, Diana, Standards of Seismic Safety for Existing Federally Owned or Leaded Buildings and Commentary, NISTIR 5382, National Institute of Standards and Technology, Gaithersburg, MD, February 1994.

H.J. Degenkolb Associates, Engineers and Rutherford \& Chekene, Consulting Engineers, Evaluation and Strengthening Guidelines for Federal Buildings Identification of Current Federal Agency Problems, NIST GCR 649, National Institute of Standards and Technology, Gaithersburg, MD, March 1994.

H.J. Degenkolb Associates, Engineers and Rutherford \& Chekene, Consulting Engineers, Evaluation and Strengthening Guidelines for Federal Buildings Assessment of Current Federal Agency Evaluation Programs and Rehabilitation Criteria and Development of Typical Costs for Seismic Rehabilitation, NIST GCR 94-650, National Institute of Standards and Technology, Gaithersburg, MD, March 1994. 


\section{Support Implementation of Executive Order 12699}

Principal Investigator:

Diana Todd

Structures Division

301-975-5296

\section{Sponsor:}

Federal Emergency Management Agency

Mitigation Directorate

\section{Objective}

To support implementation of Executive Order 12699 on seismic safety of new Federal construction by providing technical assistance when requested by affected agencies.

\section{Problem}

Affected agencies may lack knowledge within their staffs on seismic design and construction principles, and content and use of building codes.

\section{Approach}

BFRL, upon request from a Federal agency will:

- provide a speaker knowledgeable on EO 12699 and implementation issues for meetings in the Washington, DC area,

- provide multiple copies of existing literature published by BFRL supporting implementation of the Order,

- review proposed implementation procedures or regulations for appropriateness of technical content, and

- duplicate and disseminate materials produced by affected agencies for purposes of informationsharing with other agencies (only at request of the producing agency).

This work will help ensure uniform implementation of the Order, thereby reducing the seismic risk in this country.

\section{Recent Results}

Melvyn Green and Associates, Informal report to sponsor, "Analysis of Code Change Proposal B16993 to the Standard Building Code Seismic Provisions," September 1993.
Technical Assistance to FEMA Project Officer (Existing Buildings)

\author{
Principal Investigator: \\ Diana Todd \\ Structures Division \\ 301-975-5296
}

\section{Sponsor:}

Federal Emergency Management Agency

Mitigation Directorate

\section{Objective}

To provide technical review of FEMA-funded projects for the development of design and construction guidance documents for seismic rehabilitation of existing buildings.

\section{Problem}

In FY 1994, FEMA has requested BFRL's assistance to review:

- Development of Guidelines for Seismic Rehabilitation by BSSC/ATC/ASCE,

- An update of FEMA 156 and 157, Typical Costs of Seismic Rehabilitation of Buildings, and - A modification of FEMA 227, A Benefit-Cost Model for the Seismic Rehabilitation of Buildings, to be applicable to Federal buildings.

\section{Approach}

During FY 1994, BFRL staff will participate in FEMA's proposal evaluation panels, advisory panel meetings, users' workshops, and selected technical seminars and meetings. BFRL will review draft and final project products.

The effort provides FEMA with an independent technical review of the work of their contractors.

\section{Recent Results}

- Reviewed draft "Guidelines for Seismic Rehabilitation."

- Tested computer model of "Benefit-Cost Model for the Seismic Rehabilitation of Buildings." 
Secretariat U.S.-Side Panel on Wind and Seismic Effects

Principal Investigator:

Noel J. Raufaste

BFRL Headquarters

301-975-5905

Sponsor:

National Institute of Standards and Technology and 10 Federal Agencies

\section{Objective}

To provide the U.S. Secretariat of the U.S.-Japan

Panel on Wind and Seismic Effects.

\section{Problem}

Loss of life and property result from insufficient knowledge to adequately design and construct building and other structures cost-effectively against high winds and seismic loads. Improved mitigation practices are in the early stages of development. Technical collaborations between experts in the United States and Japan need to continue to realize improvements in natural hazard mitigation practices. Such improvements are possible through joint working relations with experts from the United States and Japan through performing joint research projects and exchanges of research personnel, technical data and information, and research equipment. This Panel is part of the U.S.-Japan Natural Resources Development Program under the aegis of the U.S.-Japan Cooperative Science Program of 1961.

\section{Approach}

1. Planning U.S.-side (18 Agency membership) through hosting two U.S. Panel's domestic meetings and managing the U.S. delegation's technical contributions to Annual Joint Meetings.

2. Planning and coordinating technical activities of the 10 Task Committees.

3. Maintaining liaison with U.S. and Japan Panel Members and other experts associated with the Panel's activities.

4. Planning Japan Panel Member visits to the U.S. under the auspices of the Panel, e.g., post disaster investigations, special studies, data gathering.

5. Preparing and distributing annual Panel proceedings, periodic activity reports, and other materials as appropriate to U.S.-side Panel and Task Committee Members.

The Panel provides the vehicle to exchange technical data and information on design and construction of civil engineering lifelines, buildings, waterfront, and coastal structures. The data produced by the Panel influences on-going structural engineering research and contributes to the revision and creation of U.S. building codes and standards.

\section{Recent Results}

lijima, T., Wind and Seismic Effects -- Proceedings of the 25th Joint Panel Meeting, TM 3217, Public Works Research Institute, Tsukuba, Japan, 1993. 
STRUCTURES

DIVISION

\section{STRUCTURAL BEHAVIOR}




\section{Noise-Induced Chaos and Chaotic Transport}

\author{
Principal Investigator: \\ Emil Simiu \\ Structures Division \\ 301-975-6076
}

Sponsor:

Office of Naval Research

Ocean Engineering Division

\section{Objective}

To develop measures of the probability of exit from a safe region for multistable stochastic systems.

\section{Problem}

Multistable stochastic systems may experience jumps from a safe to an unsafe region of phase space. Safe design requires knowledge of probability of occurrence of jumps and conditions guaranteeing that jumps do not occur.

\section{Approach \\ During FY 1994, BFRL staff will extend and apply to multistable stochastic systems criteria on necessary conditions for jumps originally developed for deter- ministic chaotic systems.}

The generalized Melnikov junction will be used to obtain lower bounds on probabilities of exit from a preferred region of phase space.

Recent Results

New project.

\section{Automated Construction Site Me- trology}

\author{
Principal Investigator: \\ William C. Stone \\ Structures Division \\ 301-975-6075
}

\section{Sponsor:}

National Institute of Standards and Technology

\section{Objective}

To develop a standardized system by which the real time position and orientation of any object on a construction job site can be ascertained.

\section{Problem}

Needed is a standardized construction site metrology system by which the real time position and orientation of any object on the job site -- including materials, personnel, and machinery -- can be ascertained and made available to a remote management office by means of telepresence and virtual reality interfaces. Current and emerging technologies for construction site metrics suffer from substantial limitations such as line-of-sight data acquisition requirements and severe limitations on resolution.

For some years now, a new construction site positioning system, based on the use of two or three scanning laser broadcasters and a receiving "wand" has been under development. This system shows promise for initial site metrology at construction sites where line-of-site requirements between the broadcast units and the receiver can be met. In such cases this system is positioned to replace traditional total-station surveying systems for precision measurement. However, there are many situations for which the line-of-sight requirement cannot be reasonably met ... e.g., where constructed members and natural obstacles obscure the laser transmission path. A completely alternative means of site positioning is afforded by the use of the GPS (Global Positioning System) satellite network in which local coordinates may be acquired using inexpensive hand held units. However, this system also has limitations. In general, it circumvents lineof-site problems (since the locating mechanism is situated "overhead") but it does suffer from a lack of resolution (10 $\mathrm{m}$ accuracy is the norm for a hand held unit) and does not penetrate well through overhead obstacles (e.g., a building roof).

\section{Approach}

During FY 1994, BFRL will construct an LPS (Local Positioning System) which takes the fundamental operational principles of GPS and applies them to 
site positioning by switching to the use of operational frequencies in the vicinity of $50 \mathrm{khz}$. Such waves should be able to penetrate through most building materials and provide a high degree of accuracy from a hand held system. The basic approach involves the use of four local site transmitters, each broadcasting in the $50 \mathrm{khz}$ range and each synchronized by means of a portable atomic clock. Three of these units provide wave propagation delay time distancing needed for triangulation while the fourth provides the time sync. Each unit is connected to the atomic clock by means of equal length digital fiber optic lines. This insures that each transmission unit is synchronized to within one nanosecond. The theoretical accuracy that could be achieved by such a system is in the sub-millimeter regime, with error being generated by interference from constructed objects. The degree of this error, and its frequency dependence will be the primary variables in the program.

The results of this project are expected to improve the efficiency and international competitiveness of the U.S. construction industry through the development of guidelines, standards, and prototype hardware for the accurate remote sensing and positioning of objects on construction job sites.

Recent Results

New project.

\section{High Performance Concrete}

\author{
Principal Investigator: \\ Nicholas Carino \\ Structures Division \\ 301-975-6063 \\ James R. Clifton \\ Building Materials Division \\ 301-975-6707
}

\section{Sponsor:}

National Institute of Standards and Technology

\section{Objective}

To develop guidelines for the formulation, evaluation, and engineering applications of high-performance concretes.

\section{Problem}

After many years of relatively slow advances in concrete technology, a worldwide awareness has arisen that the performance of concrete in areas such as ease of placement, strength development and durability can be significantly improved. This is evident from the program plans of the Euro-International Committee for Concrete (CEB) and the high level of concrete research activities in Japan, Norway, and Canada. In the United States, programs such as the Concrete and Structures program of the Strategic Highway Research Program (SHRP) and the establishment of the NSF Center for Advanced Cement-Based Materials (ACBM) were in response to the need for improved concretes and the recognition of the possibility of the development of high-performance concrete (HPC). The recently completed SHRP work was of a problem-solving nature, while ACBM is of an exploratory nature. This work has not provided measurement technology and design guidelines needed for U.S. leadership in high-performance concrete technology. U.S leadership in high-performance concrete technology can be a major contributor to enhancement of the competitiveness of the nation's construction industry.

\section{Approach}

This multi-year project is aimed at advancing the understanding of the properties and long-term performance of high-performance concrete (HPC) and the development of guidelines for: 1 . the proportioning and mixing of HPC; 2 . the evaluation of the physical properties and durability of HPC; 3 . structural design with HPC; and 4. field construction with HPC. Two of the urgent needs identified are: 1. information on the mechanical and physical properties of HPC, and 2. information on the durability of HPC compared to conventional concrete. In FY 1992, BFRL initiated work on develop- 
ing a basis for standards for compressive strength testing of HPC and on determining the applicability of standard frost resistance tests for conventional concrete to HPC. These studies were completed in FY 1993.

During FY 1994, BFRL will address the applicability of standard test methods for conventional concrete to HPC and will develop test methods for determining the potential alkali-reactivity of aggregates. Laboratory studies will be carried out to determine if high-strength concrete has a sufficiently high tensile strength to resist the expansive stresses from alkalisilica reactions (ASR). The results will indicate the need for testing siliceous aggregates when used in HPC. It may be that depending on the strength of HPC, the failure criteria of ASTM tests for ASR, needs to be revised. A Cooperative Research and Development Agreement (CRADA) will be developed with W.G. Grace for the purpose of carrying out collaborations research on the mechanisms of ASR. Studies on structural properties will involve an analysis of applicability of present prediction models for shear strength and development length in high strength members. A detailed experimental program on structural properties for FY 1995 and beyond will be developed to address the knowledge gaps identified in the literature review. An external review group will be assembled to review the research plan to determine if it will meet its objectives.

\section{Recent Results}

Carino, N., Guthrie, W.F., Langergren, E.S., and Mullings, G.M., "Effects of Testing Variables on the Strength of High-Strength (90MPa) Concrete Cylinders," to be presented at the $\mathrm{ACl}$ Information Conference on High Performance Concrete, Singapore, China, October 1994.
Effects of Tendon Duct Grease Leakage on the Integrity of Prestressed Concrete Containment

Principal Investigator:

Nicholas J. Carino

Structures Division

301-975-6063

\section{L.T. Phan \\ Structures Division \\ 301-975-6077}

\author{
Sponsor: \\ Nuclear Regulatory Commission \\ Office of Reactor Regulation \\ Division of Engineering
}

\section{Objective}

To conduct a preliminary investigation of the effect of tendon duct grease on the structural performance of prestressed concrete containment.

\section{Problem}

Some prestressed concrete containment structures in nuclear power plants are constructed with unbonded tendons passing through metal ducts. To protect the prestressing steel from corrosion, the ducts are injected with a specially formulated grease. In the last 10 years, it has been found that some of this grease has leaked out of the ducts and contaminated the concrete. There is a concern as to whether the grease has a detrimental effect on the concrete and mild steel reinforcement.

Approach

During FY 1994, BFRL will perform three tasks:

1. Conduct a literature search on the effects of petroleum-based oils and greases on the performance of reinforced concrete.

2 . In cooperation with staff from the Nuclear Regulatory Commission (NRC), BFRL will visit two prestressed concrete containments sites that experience tendon duct grease leaks. In-place testing will be performed, as feasible, to identify differences between concrete with grease and concrete without grease. Samples of the grease will be obtained for chemical analysis.

3. Calculations will be performed to establish the feasibility of developing a laboratory procedure for accelerated impregnation of grease into concrete specimens.

Findings from the preliminary assessment of the potential effects of grease permeation on the performance of reinforced concrete will be provided to the sponsor. If the preliminary investigation 
reveals the potential for detrimental effects, BFRL will develop a research plan for an in-depth study into the problem.

The results from this project will provide information on whether there is a potential problem to the integrity of concrete exposed to grease filler used in post-tensioned structures.

Recent Results

New project.
Next Generation Design Standard For Wind Loads

Principal Investigator:

Richard D. Marshall

Structures Division

301-975-6071

Sponsor:

National Institute of Standards and Technology

Objective

To develop key elements of an improved wind load design standard that will make extensive use of existing databases and state-of-the-art computational methods for establishing site-specific design wind speeds and the corresponding steady and fluctuating structural loads.

Problem

There are a number of significant deficiencies in the wind load design provisions of current U.S. codes and standards. They include: 1 . basic wind speeds derived from outmoded extreme value estimation methods; 2 . exposure coefficients that cannot deal with complex wind exposures (the normal case); 3. gross simplifications of extreme pressures and loads that ignore the directional effects of wind; and 4 . no distinction between design for safety and design for serviceability.

In addition to the reliable estimation of design loads, there is an urgent need for improved methods to assess wind speeds, and thus the wind forces, in complex wind exposures following extreme events such as hurricanes. With this data, it will be possible to evaluate, with reasonable confidence, the performance of structures subjected to wind loads approaching or exceeding the design limit states.

\section{Approach}

Of major interest is the tail length of distributions that best fit a given set of annual extreme wind speeds. The widely used Gumbel distribution has an infinite tail length while physical grounds require that wind speeds be bounded. Recently, statistical methods for estimating those bounds have become available. In the case of complex wind exposures, analytical models based on fundamental principles of fluid mechanics and on certain empirical relationships offer a substantial improvement over current equilibrium boundary layer models on which sitespecific wind speeds are based. Since force and pressure coefficients are direction-dependent, directional distributions of extreme wind speeds can be handled most conveniently in combination with these coefficients. The approach will be to combine 
directional extremes for a given site with wind tunnel databases for force and pressure coefficients that most closely match the geometry and/or dynamic characteristics of the structure. Finally, the development of system reliability concepts will make it possible to match safety and serviceability criteria with given levels of risk under wind loading.

The scope of work will require a three-year effort. During FY 1994, BFRL will address estimation methods for extreme wind speeds in tropical and extratropical storms. During the second year, BFRL will address wind velocity profiles over complex terrain, wind directionality, and the incorporation of existing force and pressure coefficient databases obtained from wind tunnel tests. In the third year, system reliability concepts will be developed which will be applicable to structural safety and serviceability requirements. Serviceability criteria for wind effects are in the early stages of development by the ASCE 7 Wind Loads Task Committee.

Results of this research effort should reduce the uncertainties involved with the selection of appropriate design speeds at a given site and will allow more accurate determinations of design wind loads without the need to resort to wind tunnel model studies.

Recent Results

New project.

\section{Design Wind Load Criteria for Manufactured Housing}

\author{
Principal Investigator: \\ Richard D. Marshall \\ Structures Division \\ 301-975-6071
}

\section{Sponsor: \\ Department of Housing and Urban Development Office of Policy Development and Research Innovative Technology Division \\ Objective \\ To develop improved wind load criteria for the design of manufactured housing.}

\section{Problem}

The Manufactured Home Construction and Safety Standards (CFR Pt. 3280) were implemented in 1976. Experience in south Florida during Hurricane Andrew suggests that these wind load requirements do not adequately describe the forces that a manufactured home is likely to experience in a hurricane. To improve the wind resistance of manufactured homes in hurricane-prone areas, the Department of Housing and Urban Development issued an amendment to the Manufactured Home Construction and Safety Standards (MHCSS) which is to become effective in July 1994. However, questions remain as to the wind resistance of traditional manufactured home components and assemblies, the adequacy of the MHCSS wind load requirements in non-hurricane areas, and the degree of windstorm protection provided by traditional anchoring systems.

\section{Approach}

During FY 1994, BFRL will perform the following tasks:

1. Assess the resistance of selected manufactured home structural connections and assemblies to wind loading, and evaluate the state-of-the-art for the design and testing of cladding for wind resistance. 2. Assess the significance of tornadoes as a wind load design consideration, taking into account current statistics on diameter, path length and distribution of wind speed. The distribution of extreme speeds proposed for the 1995 revision of ASCE 7 (Minimum Design Loads for Buildings and Other Structures) will be considered as well.

3. Identify alternatives to the current industry practice of supporting manufactured homes on unbonded, open-cell concrete masonry units and relying on shallow soil anchors to provide adequate resistance against sliding and uplift. 
The results of this research will incorporate current information on pressure coefficients and the distribution of extreme winds in the United States. The resulting design load criteria should lead to improved safety and performance of manufactured homes in extreme wind events.

\section{Recent Results}

Marshall, Richard D., Manufactured Homes Probability of Failure and the Need for Better Protection through Improved Anchoring Systems, NISTIR, National Institute of Standards and Technology, Gaithersburg, MD (to be published mid-1994).

Marshall, Richard D., Wind Load Provisions of the Manufactured Home Construction and Safety Standards - A Review and Recommendations for Improvement, NISTIR 5189, National Institute of Standards and Technology, Gaithersburg, MD, May 1993.
Calibration of Marshall Compaction Hammers

Principal Investigator:

Harry Shenton

Structures Division

301-975-6067

\section{Sponsor:}

Federal Highway Administration

Office of Engineering and Highway Operations

Research and Development Pavements Division

Objective

To evaluate the effectiveness of the recently developed NIST/BFRL Marshall hammer calibration system using "production" equipment at state or federal highway agency laboratories.

\section{Problem}

The Marshall test procedure is currently used by most state and local highway agencies for hot mix asphalt design. The results of many round-robin mix exchange programs have shown there is a wide variability in the mix design parameters when a given mix is compacted with different compaction hammers. Much of the scatter in Marshall test results is attributed to compaction hammer related variables, such as pedestal support, hammer alignment, hammer mass, and friction. The variability of test data implied the need for a calibration procedure for Marshall compaction hammers.

Approach

A calibration system was recently developed at BFRL with support from the Federal Highway Administration. The system has been demonstrated in a laboratory evaluation program and shown to reduce the scatter in Marshall test results by up to $60 \%$ (based on standard deviation and spread of test results). The next phase in development is to demonstrate the calibration system using field equipment.

Twelve field sites have been invited and agreed to participate in the study. The sites are located in Maryland, Virginia, West Virginia, Delaware, and Washington, DC. During FY 1994, BFRL will visit each site twice. During the first visit, two sets of four uncalibrated Marshall specimens will be prepared using the standard compactive effort of 50 and 75 blows, respectively. Diagnostic machine data will also be collected using the calibration system during the first visit. During the second visit, the site machine will be calibrated and the number of blows needed to achieve the "standard" 50 or 75 blow cumulative impulse established. Two sets of 4 calibrated Marshall specimens will be 
prepared using the number of blows determined in the calibration procedure. Bulk density, percent air voids, stability and flow will be measured for all calibrated and uncalibrated specimens. Results will be compared to determine the reduction in variability of the data for the calibrated specimens. Minor modifications or enhancements to the calibration device/system will be undertaken as part of this effort, prior to the start of the field evaluation program.

The results of this work are expected to provide more uniform asphalt pavement designs and higher quality pavements.

\section{Recent Results}

Shenton III, H.W., Cassidy, M.M., Spellerberg, P.A., and Savage, D.A., A System for Calibration of the Marshall Compaction Hammer, NISTIR 5338, National Institute of Standards and Technology, Gaithersburg, MD, January 1994.
Building and Fire Code Liaison

Principal Investigator:

Diana Todd

Structures Division

301-975-5296

\section{Sponsor:}

National Institute of Standards and Technology

Objective

To monitor activities of the three major model codes, for early alerting BFRL researchers of topics meriting BFRL input.

\section{Problem}

The results of much of BFRL's work eventually finds its way into common practice through building codes. BFRL wishes to expedite this process.

\section{Approach}

The primary codes to be monitored are ICBO's Uniform Building Code, BOCA's National Building Code, and SBCCl's Standard Building Code. In order to identify issues of concern to BFRL and opportunities for BFRL action. During FY 1994, BFRL will develop personal contacts at each of these organizations, review their periodicals, monitor proposed code changes, and attend major meetings of each group per year.

By monitoring the concerns of the code bodies and the trends in evolution of the codes, BFRL can become aware of opportunities to 1 . rapidly transfer BFRL research results into common practice, 2. contribute to the improvement of codes by providing testimony pertinent to proposed changes, and 3 . become aware of the needs of the user community to plan more effective BFRL research programs.

Recent Results

New project. 
BUILDING MATERIALS

DIVISION

\section{CONCRETE}




\section{Microstructure-Property Relation- ships in Random Porous Materials}

Principal Investigator:

Edward Garboczi

Building Materials Division

301-975-6709

\section{Sponsor:}

National Institute of Standards and Technology

\section{Objective}

To develop new, more accurate simulations of microstructure, along with simulations of new properties not previously addressed and to understand microstructure-property relationships in random porous materials.

\section{Problem}

The degradation of the concrete infrastructure, worth about $\$ 6$ trillion, is a national problem. The basic physical and chemical mechanisms of degradation processes in concrete need to be understood to make scientifically sound, non-empirical service life predictions. Most of the degradation processes that affect cement-based materials involve microstructure-property relationships for properties like diffusivity, permeability, and elastic moduli. Fundamental computer-based models are necessary to quantitatively relate such properties to the complex microstructure of cement-based materials. Quantitative relationships will serve to put existing and future standard test measurements of transport and other properties on a firm theoretical foundation.

\section{Approach}

During FY 1994, BFRL will use a digital-image-based approach and Monte Carlo techniques to simulate the formation of cement-based and ceramic materials at the fundamental level. Using finite element and finite difference techniques, coupled with fast conjugate gradient solvers, BFRL researchers will develop software to simulate and compute physical processes and parameters. These new microstructure and property simulation algorithms feed into other modeling projects and are focused on the solution of specific problems. This work is expected to advance the understanding of the mechanism of concrete degradation at the microstructural level.

\section{Recent Results}

Garboczi, E.J., "Cellular Automaton Simulations of Cement Hydration and Microstructure Development," Modeling and Simulation in Mater. Sci. and Eng., July 1994.

Garboczi, E.J., "Microstructure and Transport Properties of Concrete," in RILEM Technical Com- mittee 116 State-of-the-Art Report Permeability of Concrete as a Criterion of Its Durability, E \& F.N. Spon., London, 1994.

Garboczi, E.J., "Elastic Moduli of Interfacial Zone in Concrete, as a Function of Distance from the Aggregate Surface, Computed for the First Time," (in preparation).

Garboczi, E.J. and Snyder, K.A., "Three-Dimensional Percolation Studies of Cracks and Other Defects Carried Out Successfully," (in preparation). 


\section{Transport Properties of High- Performance Concrete}

Principal Investigator:

Nicos Martys

Building Materials Division

301-975-5915

\section{Sponsor:}

National Institute of Standards and Technology

\section{Objective}

To develop analytical and simulation models that predict transport properties of High-Performance concrete (HPC), perform experiments that validate these models, and develop improved test methods for measuring the important transport properties of HPC.

\section{Problem}

Before reliable service life predictions can be made for HPC, a fundamental understanding of the mechanisms controlling degradation of HPC is needed. Many of the degradation processes will likely be controlled by the transport properties of HPC. Due to the anticipated small pore sizes in HPC, the primary transport properties should be diffusion and capillary flow. Although diffusion in HPC has been investigated, there does not exist a comprehensive body of work that encompasses all the important factors controlling transport in HPC. In addition, no reliable test methods exist to measure the material properties (e.g., permeability, diffusion, and capillarity) that control invasion of HPC. Experimental methods and standard procedures are needed to form a basis for reliable characterization of the transport properties of HPC.

\section{Approach}

During FY 1993, BFRL studied the ingress of liquids and gases in HPC by computer simulation and by experimental studies. BFRL researchers examined basic principles of single fluid transport and hydrodynamic dispersion in porous media. Permeability of microstructural models and three-dimensional images generated by $\mathrm{x}$-ray tomography was studied. Dispersion as a function of pellet number also was examined including the role of capillary effects in fluid invasion of porous media.

During FY 1994, BFRL will analyze the knowledge base on transport properties of HPC, the reliability of models for predicting the transport properties and the reliability of test methods for measuring the transport properties. Also, plans for creating a transport laboratory, based on fundamental principles, for characterizing the transport properties of HPC will be developed. This phase of the research will result in a summary of state of knowledge of transport properties of HPC including research needed to improve prediction and measurement of transport properties and durability of HPC.

Microstructural models, based on images of authentic microstructures, will be used in simulation models to calculate transport properties at a microscopic (or pore size) scale as related to behavior on the macro scale (e.g., mortar and concrete). The models will be validated by experimental studies. The adequacy of existing test methods for measuring the transport properties of HPC will be assessed.

\section{Recent Results}

Nolle, C.S., Koiller, Belita, Martys, Nicos, and Robbins, Mark O., "Morphology and Dynamics of Interfaces in Random Two-Dimensional Media," Physical Review Letters, v71, no. 13, pp 20742077, The American Physical Society, September 27, 1993.

Schwartz, L.M., Martys, N., Bentz, D.P., Garboczi, E.J., and Torquato, S., "Cross Property Relations and Permeability Estimation in Model Porous Media," Phys. Review E, v48 (6), pp 4584-91, 1993. 


\section{Integrated Knowledge System for High-Performance Concrete}

Principal Investigator:

James R. Clifton

Building Materials Division

301-975-6707

\section{Sponsor:}

National Institute of Standards and Technology

Objective

To develop an integrated knowledge system for high-performance concrete (HPC) which will be a model for other high-performance construction materials (HPCMs).

Problem

A major requirement for exploiting the benefits of HPCMs is making reliable information about these materials (composition, properties, and performance) available to producers, users and other decision makers. Owners, designers, and contractors must have access to reliable information about HPCMs that can be quickly and economically retrieved. This can be accomplished by organizing the information in an integrated computer-based knowledge system. An integrated knowledge system for HPCMs should consist of databases integrated with mathematical models and expert opinion, to assist engineers and contractors in using the most reliable and advanced knowledge for selecting and using HCPMs.

\section{Approach}

During FY 1994, BFRL will plan and conduct a symposium in collaboration with the private and public sectors. The symposium will introduce Federal agencies and the private sector to the purpose, approach, and benefits of developing integrated knowledge systems. Also, the symposium will initiate planning for the development of an integrated system for HPC, including its conceptual design and architecture.

The conceptual design will be planned in FY 1994 and, as far as possible, the architecture for a distributed integrated knowledge system for HPC will be designed. In future years, a demonstration model knowledge system model will be developed for HPC, then a demonstration prototype system, followed by an operational system and, finally, the integrated knowledge system for HPC will be implemented. The system will facilitate the making of sound decisions concerning HPC.
Computation of the Micro-Mechanical Properties of Cement-Based Materials

\author{
Principal Investigator: \\ Edward J. Garboczi \\ Building Materials Division \\ 301-975-6708
}

Sponsor:

National Institute of Standards and Technology

\section{Objective}

To develop and apply numerical techniques to compute and understand the micro-mechanical properties of high-performance concrete.

\section{Problem}

The complex microstructure of concrete, from cement particle length scales up to coarse aggregate length scales, results in complex mechanical behavior of this composite material. New, high performance concrete materials pose new problems of possible brittleness, the effect of flaws, and the role of the interfacial zone in reducing strength. These and other mechanical behaviors need to be understood at the microstructural level.

\section{Approach}

During FY 1994, BFRL will use digital-image-based finite element models for elastic problems to first consider the elastic properties of the cement paste matrix. One interface will then be added, and the elastic properties of the interfacial zone cement paste computed, for a range of parameters like water:cement ratio and cement particle size distribution. This data will be incorporated into higher-order models of mortar and concrete, where the cement paste will be treated as a continuum material, but with properties that change through the interfacial region. Strength of the different phases and of the interfacial zone can then be specified, and fracture simulations performed. These computer simulation methods will allow the mechanical interactions between the density packed aggregates in concrete to be theoretically studied, in a realistic way, for the first time.

\section{Recent Results}

New project.

Recent Results

New project. 
Quantitative X-Ray Powder Diffraction Analysis of Clinker and Cement

Principal Investigator:

Paul Stutzman

Building Materials Division

301-975-6715

Sponsor:

National Institute of Standards and Technology

Objective

To develop application and testing methods of quantitative $\mathrm{x}$-ray powder diffraction routines for determining phase abundance of clinker and cement and develop a standard test method for sample preparation and analysis.

\section{Problem}

Improved methods of characterization of clinker and cement are necessary for advances in cement and concrete technology and to aid in the prediction of the performance of concrete-making materials and concrete. X-ray powder diffraction is commonly used for phase analysis and is the only method for quantitative phase abundance analysis of finegrained materials. Currently a standard method for quantitative analysis by $\mathrm{x}$-ray powder diffraction does not exist, and no guide is available for powder diffraction analysis of clinker and cement.

\section{Approach}

Clinker and cement diffraction patterns are difficult to analyze quantitatively because of differences in mass absorption coefficients of the constituent phases and severe overlapping of most of the intense diffraction peaks. The remaining very low intensity peaks are subject to counting and measurement errors. Current techniques require the use of an internal standard to correct for absorption effects, decomposition of peak overlaps, measurement of peak area, and use of calibration curves relating peak area ratios to concentration. An alternative procedure currently being tested is a whole-pattern fitting in which the entire diffraction pattern is used instead of individual peaks. The main advantage of this method is that individual peaks do not need to be resolved. The CMRL Reference Cements and ICMA round robin clinkers will be analyzed in this study. From these analyses a guide will be developed providing details on sample preparation, extraction methods, qualitative and quantitative analytical methods.

The results of this research will be provided to ASTM Task Group C01.23.01 on quantitative x-ray powder diffraction methods as a potential test method or recommended practice for phase abundance analysis of clinker and cement.

Recent Results

Stutzman, P., Quantitative X-Ray Powder Diffraction Methods for Clinker and Cement, NISTIR 5403, National Institute of Standards and Technology, Gaithersburg, MD, April 1994. 


\section{Factors Affecting the Performance of Beneficiated Fly Ash in Concrete}

Principal Investigator:

Paul E. Stutzman

Building Materials Division

301-975-6715

James R. Clifton

Building Materials Division

301-975-6706

\section{Sponsor:}

National Institute of Standards and Technology

\section{Objective}

To investigate relationships between compositional and microstructural features of fly ash and investigate the performance of fly ash in concrete.

\section{Problem}

Fly ash is a by-product of the combustion of coal in power plants. A small portion of the large amount of fly ash generated each year is used in concrete in which it serves a useful function as a partial replacement for portland cement. The rest has to be disposed of at a significant cost to the power plant companies and to the detriment to the environment. Liskowitz and collaborators at the New Jersey Institute of Technology (NJIT) have recently shown that the performance of fly ash in concrete, as indicated by strength development and resistance to sulfuric acid, can be significantly improved by either or both of optimal control of the combustion conditions in the boiler and beneficiation of the ash in an air separator which removes the largest particles. If these findings can be shown to be of general validity, they should be of national importance because of new options made available for the production of high-performance concrete and the advancement of "green" concrete technology by facilitating: 1, a reduction in the energy embodied in concrete, 2 . reduction in the amount of portland cement needed in concrete with an associated reduction in the amount of carbon dioxide emitted in cement manufacture, and 3 . reduction in the volume of fly ash for disposal. This requires understanding of the relationships among chemical and physical properties of ash and its performance in concrete so that the technical basis for predicting the performance of concrete containing beneficiated fly ash can be established.

\section{Approach}

During FY 1994, BFRL will develop an improved understanding of the mechanisms and kinetics of reactions between portland cement and fly ash; identify the composition of the reaction products; and develop relationships between proportions of portland cement and fly ash, water-to-cement ratio, degree of reaction, and the resulting microstructural features such as pore structure and phase compositions. This research will involve the use of scanning electron microscopy, quantitative $x$-ray powder diffraction, and chemical analyses by $x$-ray microanalysis. Simulation modeling will be performed to develop an understanding of the resulting microstructures which will be important in predicting the long-term performance of portland cement-fly ash concretes.

Portland cement-fly ash mortars prepared by NJIT will be characterized in terms of their microstructures, phase, and chemical compositions. Also, the portland cements and fly ashes will be characterized. Plans for experimental studies including the development of accelerated durability tests needed to predict the durability of the portland cement-fly ash concretes will be performed.

Recent Results

New project. 
Performance Criteria for Long Lived Concrete for Radioactive Waste Storage

Principal Investigator:

James R. Clifton

Building Materials Division

301-975-6707

\section{Sponsor:}

U.S. Nuclear Regulatory Commission

Waste Management Branch

\section{Objective}

To develop performance criteria for selecting concretes to be used in constructing structures with 500 year safe lives for storing low-level radioactive wastes (LLW).

\section{Problem}

The U.S. Nuclear Regulatory Commission (NRC) is responsible for developing a strategy for storing low-level radioactive wastes. According to one approach, the radioactive wastes would be disposed of in concrete vaults which are either buried in the earth or constructed above ground and covered with earth. A safe service life of 500 years is required for the vaults which may be left unattended for most of their life. Based on consideration of the past durabilities of concrete, it appears possible that concrete can be designed to give a service life of at least 500 years in favorable environments. The present basis for selecting concretes and their constituents needs to be further advanced to increase the likelihood that concretes could achieve safe lives of 500 years.

\section{Approach}

In this multi-year project, the development of performance criteria involves many of the steps included in ASTM E 632, "Standard Practice for Developing Accelerated Tests to Aid Predictions of the Service Life of Building Components and Materials."

Accelerated testing along with mathematical modeling of the rate of deterioration of concrete is being used in developing a basis for making service life predictions.

The work carried out from FY 1988 through FY 1993 included:

1. Identification of likely degradation processes and analyses of their mechanisms and potential rates.

2. Development of mathematical models of transport processes in concrete involving moisture, chloride ions, sulfate ions, and acids.

3. Modeling of chloride and sulfate ion diffusion in concrete.
4. Evaluation of test methods for determining the potential alkali-reactivity of aggregates.

5. Evaluation of the reliability of tests for predicting the sulfate resistance of concrete exposed to ground water.

6. Performing evaluations to understand the mechanism of sulfate attack on buried concrete.

During FY 1994, BFRL will complete studies on the mechanism and kinetics of sulfate attack on buried concrete. Also, BFRL will complete its research on the effects of cracks on the hydraulic conductivity of concrete. The research will lead to recommended performance criteria for use in selecting concrete for constructing LLW disposed vaults with a service life of 500 years.

\section{Recent Results}

Pommersheim, J.M. and Clifton, J.R., "Expansion of Cementitious Materials Exposed to Sulfate Solutions," Scientific Basis for Nuclear Waste Management Materials Research Society, in press.

Clifton, J.R., Bentz, D.P., and Pommersheim, J.M., Sulfate Diffusion in Concrete, NISTIR 5361, National Institute of Standards and Technology, Gaithersburg, MD, 1994.

Pommersheim, J.M. and Clifton, J.R., Sulfate Attack of Cementitious Materials: Volumetric Relations and Expansions, NISTIR 5390, National Institute of Standards and Technology, Gaithersburg, MD, 1994. 


\section{Concrete Degradation Computer Code for LLW Performance Assessments}

\author{
Principal Investigator: \\ James R. Clifton \\ Building Materials Division \\ 301-975-6707 \\ Sponsor: \\ Nuclear Regulatory Commission \\ Waste Management Branch
}

\section{Objective}

To develop computer models of concrete degradation for use by the Nuclear Regulatory Commission in the assessment of the performance of underground reinforced concrete structures designed to contain low-level radioactive wastes (LLW).

\section{Problem}

Reinforced concrete has been proposed by many states and compacts of states to construct engineered barriers for disposing LLW.

To meet the requirements of the Federal regulations for LLW disposal, 10 CFR 61, the concrete structures must demonstrate specific durability and performance attributes for periods of 300 to 500 years. The Nuclear Regulatory Commission (NRC) is responsible for determining if proposed disposal facilities meet the requirements set forth in 10 CFR 61. To provide guidance to state and compact LLW personnel in designing LLW disposal concrete structures, the Nuclear Regulatory Commission is developing a Branch Technical Position/Regulatory Guide for LLW performance assessments. The assessment will include the use of models for predicting the effect of degradation processes on the properties of the concrete used in constructing LLW disposal vaults, The models need to be able to predict changes in the hydraulic properties of the concrete during 300 to 500 years of service.

\section{Approach}

BFRL is developing computer models to predict the service life of concrete used in the construction of underground concrete vaults for LLW disposal. The degradation processes being addressed include corrosion of reinforcement, sulfate attack, leaching, and acid attack. Existing models are being analyzed to determine if they are reliable or can be satisfactorily modified. If necessary, new models will be developed. A peer review committee has been established to critique the development of the computer code. The interactive computer code under development will be capable of being run on a micro-computer by the NRC staff.

A manual will be prepared and training exercises will be conducted to assist NRC staff and the planners and designers of LLW disposal sites in using the computer models. The final report will give recommendations on further model developments and their validations.

\section{Recent Results}

Clifton, J.R., Pommersheim, J.M., Snyder, K.A., and Martys, N., Concrete Degradation Computer Code for LLW Performance Assessment, NISTIR, National Institute of Standards and Technology, Gaithersburg, MD (to be published). 
BUILDING MATERIALS DIVISION

\section{COATINGS}




\section{Service Life of Coating Systems}

\author{
Principal Investigator: \\ Jonathan W. Martin \\ Building Materials Division \\ $301-975-6717$ \\ Tinh Nguyen \\ Building Materials Division \\ 301-975-6718
}

\section{Sponsor:}

National Institute of Standards and Technology

\section{Objective}

To develop an experimental protocol and theoretical model for quantitatively predicting the service life of coating systems exposed in typical exposure environments in the field and the laboratory; identify methods for quantitatively characterizing exposure conditions and; demonstrate how field and laboratory exposures are related.

\section{Problem}

The inability to control or adequately characterize temporal changes in the factors comprising the weather is one of the major challenges which will have to be overcome in establishing a connection in the degradation response of polymeric materials exposed in the laboratory and the field. Although the number of weathering factors is large, only three factors, UV-irradiation, wetness, and temperature, are considered to be of prime importance in most nonindustrial environments.

Solar UV-radiation causes large economic losses in materials, humans, and other biological systems. For building materials, these losses are particularly high for materials having large surface areas and large infrastructural inventories, like organic coatings, asphalt, vinyl and aluminum siding, wood, and roofing membranes. At present, no national or international standards exist for monitoring or modeling the degradation of materials resulting from UV-irradiation. Such standards are necessary for predicting the service lives of polymeric systems and producing consistent results from one laboratory to another.

\section{Approach}

In FY 1993, mathematical models were developed for modeling changes in the spectral UV-irradiance in the field over time. These models were verified using spectral UV-irradiance data collected by the Smithsonian Institution at their Rockville, MD site. BFRL's research revealed that temporal changes in spectral UV-irradiance data can be modeled using time series analysis. The irradiance between 295 and $320 \mathrm{~nm}$ (the limits of the Smithsonian Institution's data) may be predicted from measurements at two wavelengths, and errors in monitoring spectral UV-irradiance data are small if the halfwidth of the narrow bandwidth filters used in detecting spectral UV-irradiance is $2 \mathrm{~nm}$ or less.

During FY 1994, BFRL will develop mathematical models to determine materials degradation effects of spectral UV-irradiance. The absorption and quantum yields will be determined for two coating systems as a function of wavelength and temperature. Attention will be focused on determining the range of wavelengths over which the quantum yield response is positive, the number of wavelengths required to fully specify the absorption and quantum vield spectra, and the effect on the experimental error of filters having different half-widths. Also, attention will be given to modeling the degradation of high-performance coatings exposed in corrosive environments.

Leadership will be provided in subcommittee ASTM G03.08 on Service Life Prediction and the Task Group on Environmental Characterization in the RILEM/CIB Committee on Service Life Prediction. BFRL will encourage creating an indus-

try/government consortium to expedite research on service life of coating. The results of this research are expected to improve the technical basis for selecting organic coatings in terms of their likely life under the expected service conditions.

\section{Recent Results}

Pommersheim, J., Nguyen, T., Zhang, Z, and Lin, C., Diffusion of Cations Beneath Organic Coatings on Steel Substrate, NISTIR 5102, National Institute of Standards and Technology, Gaithersburg, MD, 1993.

Nguyen, T. and Pommersheim, J., "Diffusion of Cations Along the Polymer/metal interface under an Applied Potential," Materials Research Society Proceedings, 304:15 (CC 1020 MS 1), 1993.

Nguyen, T., Hubbard, J., and Pommersheim, J., "Models for the Degradation of Organic Protective 
Coatings," Proceedings of the Durability of Building Materials and Components, S. Nagataki et al, Eds., E. and R.N. Spon publisher, Vol 1, p. 1070 (CC 1020 MS 1), 1993.

Nguyen, T., Bentz, D., and Byrd, E., "Measuring Water at the Polymer Coating/Hydroxylated Interface," Proc. Adhesion Society Meeting (CC 1020 MS 2), 1993.

Nguyen, T., Bentz, D., and Byrd, E., "Measuring Transport of Liquid Water Through Organic Coatings Adhered to a Substrate," ACS Proceedings, Polymeric Materials Science and Engineering 68:103 (CC 1020 MS 2), 1993.

Lechner, J.A. and Martin, J.W., "Modeling and Measuring Ultraviolet Irradiance to Predict Damage to Macromolecular Materials," Proceedings of the American Chemical Society Division of Polymeric Materials: Science and Engineering, Vol. 69 (CC 1023 MS 4l, 1993.

Martin, J.W., Lechner, J.A., and Varner, R.N., "Quantitative Characterization of Exterior Weathering Environments: UV-Radiation," Accelerated and Outdoor Durability Testing of Organic Materials, ASTM STP 1202, Warren D. Ketola and Dougias Grossman, [Eds.], American Society for Testing and Materials, Philadelphia, PA. (CC 1023 MS 4), 1993.

Martin, J.W., "Quantitative Characterization of Spectral Ultraviolet Radiation-induced Photodegradation in Coating Systems Exposed in the Laboratory and the Field," Progress in Organic Coatings 23(1): 49 (CC 1023 MS 5), 1993.

\section{Organic Coatings}

\author{
Principal Investigator: \\ Mary McKnight \\ Building Materials Division \\ 301-975-6714
}

\section{Sponsor:}

Department of the Navy

North Navy Facility Engineering Command

\section{Objective}

To develop improved procedures for selecting, using, and specifying coating systems and for transferring the technology to Department of the Navy personnel responsible for maintaining coatings on structures.

\section{Problem}

Military facilities have an estimated real property value of $\$ 300$ billion and the annual cost of coating maintenance is about $\$ 400$ million. Improved and updated criteria for the selection, specification, and use of protective coatings is needed to take advantage of new technologies and meet environmental regulations, e.g., those relating to volatile organic compound (VOC) content and heavy metal content.

\section{Approach \\ During FY 1994, BFRL will provide technical} assistance to the Navy and DoD through the TriServices Committee about low VOC coatings, prepare a chapter on the selection and use of VOC compliant coatings for a new Military Handbook on maintenance painting, assist in the implementation of these new technologies, and organize Tri-Service Protective Coatings Committee meetings. To help meet the need for new coating specifications for low VOC coatings, BFRL will continue to chair the SSPC committee on coatings and assist in the development of low $\mathrm{VOC}$ coating specifications. Military Handbook provides current information on coating of facilities which is expected to result in improved coating lifetimes, thus reducing total maximum cost, and to aid in solution of environmentally compliant systems.

\section{Recent Results}

McKnight, M.E., Chapter on Environmental Concerns for Tri-Service Paint and Protective Coatings Handbook, DoD Manual, MIL-HDBC-1110 (submitted for publication). 
McKnight, M.E. and Roberts, W.E., Coatings for Application to Moist Steel: Test Procedures to Evaluate Initial Adhesion and Cure Properties, NISTIR 6228, National institute of Standards and Technology, Gaithersburg, MD, July 1993.

\section{Standards for Abatement of Hazards from Lead in Buildings}

Principal Investigator:

Mary McKnight

Building Materials Division

301-975-6714

\section{Sponsor:}

U.S. Department of Housing and Urban Development

Office of Lead-Based Paint Abatement and Poison Prevention

Objective

To develop technical data for use in standards for abatement of hazards from lead in buildings and structures.

\section{Problem}

An estimated 57 million homes in the United States contain some lead-based paint. Although there is general guidance for characterizing the lead concentrations in paint, dust, soil, and air particulates and carrying out abatements, there are very few standard procedures that address these issues. Thus, there is a need for an overall standard practice for abating hazards from lead in buildings and related structures that would include assessment and analysis of the problem, design of abatement strategy, abatement procedures and post-abatement management procedures. An ASTM Subcommittee, E06.23, was organized to meet this need.

\section{Approach}

The approach that E06.23 is using to develop the overall practice for abatement of hazards from lead in buildings and structures is to develop the basic standards that will serve as the framework of the overall guide. These include standards for characterizing the concentration of lead in paint, dust, soil and air particulates and for carrying about abatements and hazard reduction. Once most of these basic standards are completed, they will be combined into the overall practice. An added advantage of developing individual standards for each step of the hazard-abatement process is that as soon as any standard is approved, it can be referenced in contracts and other documents. To maintain the subcommittee's viability, the approach is to continue to recruit additional knowledgeable members, to provide results of research to the appropriate task 
groups, and to form improved liaisons with other ASTM committees including D-22, D-1, E-50, E-1 and $\mathrm{D}-18$.

\section{Recent Results}

Results contributed to development of E06.23

Subcommittee on Abatement of Hazards from Lead in Building Standards:

- E1605 Terminology Relating to Abatement of Hazards from Lead-Based Paint in Buildings and Related Structures.

E1553 Standard Practice for Collection of Airborne Particulate Lead During Abatement and Construction Activities.

- E1583 Practice for Evaluating Laboratories Engaged in the Determination of Lead in Paint, Dust, Airborne Particulates, and Soil in and Around Buildings and Related Structures.

ES035 Standard Method for the Analysis of Digested Samples for Lead by Inductively Coupled Plasma Atomic Emission Spectrometry (ICP-AS), Flame Atomic Absorption (FAAS) or Graphite Furnace.

- ES036 Practice for Hot Plate Digestion of Dust Wipe Samples for the Determination of Lead by Atomic Spectrometry.

ES037 Practice for the Preparation of Dried Paint Samples for Subsequent Lead Analysis by Atomic Spectrometry.

\section{Method for Evaluation of Perfor- mance of Lead-in-Paint Measuring Devices Under Simulated Field Conditions}

\author{
Principal Investigator: \\ Mary McKnight \\ Building Materials Division \\ 301-975-6714
}

\section{Sponsor:}

U.S. Department of Housing and Urban Development

Office of Lead-Based Paint Abatement and Poison Prevention

\section{Objective}

To identify and quantify factors affecting the field performance of portable $x$-ray fluorescence (XRF) measurements of lead in paint using a range of simulated field conditions, develop a laboratory test method suitable for standardization for evaluating portable XRF devices, and write a draft standard test method for the laboratory-based evaluation of portable XRF devices and present it to ASTM E06.23 for standardization.

\section{Problem}

Recent legislation (Housing and Community Development Act of 1992, PL102-550) will phase in requirements for lead-based paint testing for all housing built before 1978 . These requirements are expected to result in several new $\mathrm{x}$-ray fluorescence (XRF) devices.

Because of both health and financial liabilities associated with these measurements, it is essential to have reliable estimates of the field performance parameters of the measurements (e.g., detection limit, precision, bias). For some XRF devices, research results have shown that estimates of these performance parameters obtained using existing laboratory evaluation procedures do not agree with estimates obtained using field-acquired data.

Currently, field studies are being used to characterize additional devices. However, field studies are limited in several ways -- sites having parameters in the necessary ranges are difficult to find; systematic variation of the parameters is usually not possible; implementation of new technologies may be impeded; and advances in the fundamental understanding 
of a measurement procedure are limited. Thus, there is a need for a laboratory-based field-simulation test protocol for the reliable evaluation of portable XRF devices which will overcome these limitations.

\section{Approach}

During FY 1994, BFRL will perform three tasks:

Task 1. Develop a mathematical model that embodies the fundamental understanding of the measurement process and the effects of the composition and configuration of a test specimen upon the measurement response. A conceptual model of the measurement process will be constructed to portray the scientific principles involved, factors likely to affect instrument response, and characterization of typical field situations in terms of these factors. A mathematical model based upon the conceptual model will be developed. The mathematical model will be used throughout the study to explore the implications of various material configurations and field conditions on instrument response and to aid interpretation of experimental results.

Task 2. Design a simulated field study and an actual field study using information obtained from the literature and other sources and from the model in Task 1. The design for the laboratory-based field simulation study will include use of model wall sections each about one meter square containing materials in configurations typical of those found in residential buildings. The laboratory experiment will be carried out in a room with temperature and humidity controls that allow the conditions to be changed as needed. The model sections will be constructed in such a way that the materials and configurations within them can be systematically changed to represent ranges of field conditions to study their effects upon instrument response. The field experiment will be conducted to determine whether the estimates of measurement variability obtained in the laboratory and field are consistent with each other.

Task 3. Upon completion of the experimental study, BFRL will develop a draft standard test method for evaluating the performance of portable XRF equipment will be written and presented to ASTM E06.23.
The results of this research are expected to aid in selecting XRF devices for field measurements of lead in paint.

Recent Results New project. 


\section{Development of Method for Leach- ing of Lead From Lead-Based Paint Through Encapsulants}

Principal Investigator:

Mary McKnight

Building Materials Division

301-975-6714

Tinh Nguyen

Building Materials Division

301-975-6718

\section{Sponsor:}

U.S. Department of Housing and Urban Development

Office of Lead-Based Paint Abatement and Poisoning Prevention

\section{Objective}

To develop test method for determining the rate of transport of lead from lead-based paint through an encapsulant coating by leaching and prepare draft standard method and present to ASTM E06.23 Subcommittee on Abatement of Hazards from Lead in Buildings for standardization.

\section{Problem}

According to a recent U.S. Department of Housing and Urban Development (HUD) report to Congress, an estimated 57 million homes in the United States contain some lead-based paint. Several abatement procedures are available for controlling the hazards associated with lead-based paint, including the use of encapsulant coatings. Encapsulants appear to have promise for providing a cost-effective abatement technique for many situations. Before these benefits can be realized, it must be established that encapsulants can provide a long-lived intact film and prevent leaching of lead from the underlying leadbased paint layer through the encapsulant to its surface. Leaching could conceivably occur when films are wetted, whether by rain, condensed water, cleaning solutions, or other liquids. Since failure to contain the lead would be unacceptable, a test method and a criterion are urgently needed to prevent coatings having poor leaching resistance from being used as encapsulants. This need has also been identified by ASTM E06.23 which is preparing a standard on encapsulant coatings.
Approach

During FY 1994, BFRL will develop a procedure for determining the rate of transport of lead through encapsulant coatings. Paint having a lead content of approximately 20 percent by mass of nonvolatile components will be applied to a suitable substrate. Upon cure of the lead-based paint, a test encapsulant will be applied and allowed to cure according to the manufacturer's directions. A vessel will be attached to the surface of the encapsulant film and an aqueous leaching solution will be placed in the vessel. Aliquots of the solution will be withdrawn at predetermined time intervals and analyzed for lead content by atomic absorption spectrometry. The following test parameters will be investigated: type of lead pigment, substrate type, curing and aging procedure for lead-based paint, encapsulants and paints, aqueous leaching solutions (weakly acidic, neutral, and weakly basic), test temperature of test. Leaching solutions will be analyzed using atomic absorption spectrometry. The cross sections of a subset of the specimens will be examined using scanning electron microscopy and $\mathrm{x}$-ray photoelectron spectroscopy for evidence of lead transport through the encapsulant. The results of this research are expected to provide a basis for establishing a performance criteria for selecting encapsulants.

Recent Results

New project. 


\section{Performance Criteria for Selection of Liquid-Coatings Encapsulants for Lead-Based Paint}

\author{
Principal Investigator: \\ Walter Rossiter \\ Building Materials Division \\ 301-975-6719 \\ Mary McKnight \\ Building Materials Division \\ 301-975-6714
}

\section{Sponsor:}

U.S. Department of Housing and Urban Development

Office of Lead-Based Paint Abatement and Poisoning Prevention

\section{Objective}

To develop preliminary performance criteria for liquid-coating encapsulants for lead-based paint in housing based on short-term laboratory testing and limited field testing and to extend the development of the preliminary performance criteria for liquidcoating encapsulants for lead-based paint to include results from long-term tests.

\section{Problem}

In a survey of the nation's housing, the U.S. Department of Housing and Urban Development (HUD) found that about 57 million homes in the United States have lead-based paint (LBP) on at least one painted surface. One way of helping to ensure that the hazards of LBP in housing are kept low is to protect the paint by a liquid-coating encapsulant. A major deterrent to the use of these encapsulants is the lack of performance standards and field performance data. Under proposed legislation, HUD will sponsor an encapsulant demonstration project in public and Indian housing. To prepare for the purchase of encapsulants for this project, HUD has an urgent need for performance criteria. Such criteria are being prepared by ASTM Subcommittee E06.23 on Abatement of Hazards from Lead in Buildings, but are unlikely to be completed soon enough to satisfy this immediate need.

\section{Approach}

This research will be performed in two phases.
Phase 1. Develop preliminary performance criteria based primarily on early age or short-term laboratory and field tests.

Phase 2. Refine the criteria and extend them to include preliminary criteria that address long-term performance.

In Phase 1, performance requirements and test procedures will be identified. On the most general performance level, encapsulants must, with an acceptable probability, ensure that the encapsulant keeps the lead in place and prevents it from contaminating the environment for the desired time. Therefore, the performance criteria must address encapsulant material characteristics that prevent release of lead such as abrasion resistance, impact resistance, leaching resistance and ability to prevent failure (e.g., peeling, flaking and chipping) of the underlying LBP layer.

The approach to be followed in developing specific requirements and procedures is:

1. Visit several field sites where encapsulants have been installed or are being considered for use to aid in the identification of specific requirements and selection of test materials.

2. Select substrate test materials and develop procedures for preparing them in a uniform and consistent manner to simulate conditions expected in the field.

3. Select encapsulants and control paints to use in testing.

4. Select appropriate test procedures using ASTM tests where appropriate and prepare test specimens.

5. Subject specimens to laboratory performance tests under conditions in which the applied stresses are at the high ends of the ranges expected under service conditions.

6. Based on the results of the laboratory tests, perform limited field testing.

Using both data sets, preliminary performance criteria will be developed. Results of the research will be provided to ASTM E06.23 to use in improving the technical bases for standards for encapsulants.

In Phase 2, the research will focus on performing long-term laboratory aging and weathering tests and 
additional field tests to provide data for verifying and improving the preliminary performance criteria.

Recent Results

New project.

\section{Long-Term Performance of Polymer-Based Encapsulants for Lead-Based Paint on Interior Walls}

\author{
Principal Investigator: \\ Mary McKnight \\ Building Materials Division \\ 301-975-6714
}

Sponsor:

Department of the Army

Engineering Housing and Service Center

\section{Objective}

To determine changes in physical, chemical, and mechanical properties of coatings, as a function of accelerated weathering exposure.

\section{Problem}

The Department of Defense (DoD) has approximately 500,000 family housing units built prior to 1978 many are likely to have some surfaces coated with lead-based paint. Based on a recent U.S. Department of Housing and Urban Development report, the DoD should obtain significant cost savings if encapsulants can be used to abate surfaces with lead-based paint as compared with removal. However, before encapsulants are widely used on DoD Facilities, it is essential that information regarding their long-term performance be available, since premature failure would be very costly. A generally applicable methodology is needed to predict the long-term performance of an encapsulant, rather than evaluating a few products. Such a methodology is required by DoD as many materials are currently being marketed as encapsulants, and these products are expected to expand in number in the future. The methodology would serve as the basis for performance-based selection criteria and life-cycle cost analyses.

\section{Approach}

During FY 1994, BFRL will develop the methodology for predicting long-term performance of leadbased paint encapsulants. This work will determine relationships between changes in physical, chemical and mechanical properties of encapsulant coatings and time of exposure in controlled environments. The results of these experiments will be used to develop methodologies for service-life prediction. The information also will be useful in developing 
selection criteria. In BFRL's experimental study, three generic types of commercial, polymer-based encapsulants, and a latex paint, will be subjected to five environmental conditions, selected from a combination of three temperatures, $10^{\circ} \mathrm{C}, 25^{\circ} \mathrm{C}$, and $50^{\circ} \mathrm{C}$, and three relative humidities. Performance data on properties, which are important to performance as an encapsulant, such as resistances to cracking, peeling (adhesion), damage upon impact, and abrasion; glass transition temperature; weight stability (thermogravimetric analysis), modulus, and chemical stability (spectroscopy) and will be obtained. The results of this work are expected to provide a methodology for predicting service life of encapsulants that can be used in performing cost-benefit analysis prior to selection of an encapsulative abatement strategy.

Recent Results

New project.

\section{Technical Assistance on Lead- Based Paint Issues}

\author{
Principal Investigator: \\ Mary McKnight \\ Building Materials Division \\ 301-975-6714
}

\section{Sponsor:}

U.S. Department of Housing and Urban Development

Office of Lead-Based Paint Abatement and Poisoning Prevention

\section{Objective}

To provide technical assistance on lead-based paint material-related issues and measurement procedures.

\section{Problem}

The U.S. Department of Housing and Urban Development (HUD) receives many requests for technical assistance on issues about abating hazards from lead-based paint in housing. Some of these requests address material properties and performance of paints and coatings and material-related measurement procedures.

\section{Approach}

During FY 1994, BFRL will provide, as needed, technical assistance on topics such as the performance of paints and coatings, the abatement of hazards from lead-based paint, measurement of lead concentrations in paint films, and other materialrelated issues.

\section{Recent Results}

As requested by HUD, observed and commented on demonstration of new portable XRF devices for lead in paint measurements at New York City Housing, Preservation and Development Office. 
BUILDING MATERIALS DIVISION

\section{QUALITY ASSURANCE}




\section{Cement and Concrete Reference Laboratory}

\author{
Principal Investigator: \\ James H. Pielert \\ Building Materials Division \\ 301-975-6704
}

\section{Sponsor:}

American Society for Testing and Materials (ASTM)

Department of the Army

Corps of Engineers

\section{Objective}

To inspect cement and concrete testing laboratories, distribute proficiency test samples, and support the voluntary standards development process.

\section{Problem}

The infrastructure represents a substantial portion of the nation's wealth and the cost of construction of facilities amounts to about 10 percent of the U.S. Gross Domestic Product. Over $\$ 4$ billion of hydraulic cement is produced in the United States each year with the value of the concrete construction estimated to be in the order of $\$ 20$ billion. Standardization of testing to enhance the reliability of quality assurance measurements is of paramount concern. The productivity of the testing community in the cement and concrete fields can be increased by the use of correct procedures and apparatus which reduce testing errors and provide a sound basis for the acceptance of cement on mill certificate. Efficient use of long-established construction materials is facilitated by dependable quality assurance programs.

\section{Approach}

With the support of ASTM Research Associates working under BFRL supervision, services are provided to public and private cement and concrete testing laboratories on a voluntary basis. These services include the laboratory inspection program (LIP) and the distribution of proficiency test samples. Equipment and procedures used in performing conventional quality assurance tests are evaluated for conformance to applicable national standards. Related test apparatus is checked with inspection equipment calibrated by NIST personnel. Proficiency test samples of portland cement, pozzolan, concrete, blended cement and masonry cement are distributed at regular intervals to obtain information on laboratory performance. Additionally, technical studies are conducted, often in conjunction with other NIST Laboratories, in subject areas related to these programs.

Specific products of this work include: 1 . detailed inspection reports; 2 , report on each round of proficiency sample testing; 3 . input to the work of standards committees such as draft standards and precision data; and 4. reports on results of technical studies. Findings from BFRL technical reports, papers in outside journals, and oral presentations are used by the cement and concrete community in product improvement.

Recent Results

Kolos, R.M., "Conformity Data from the CCRL Laboratory Inspection Program," ASTM Cement, Concrete, and Aggregates (pending).

During FY 1993, over 250 cement and concrete laboratories were inspected, over 3000 proficiency samples were distributed, and corresponding test reports were issued. 


\section{AASHTO Materials Reference Laboratory}

\author{
Principal Investigator: \\ James H. Pielert \\ Building Materials Division \\ 301-975-6704
}

\section{Sponsor:}

American Association of State Highway and

Transportation Officials (AASHTO)

\section{Objective}

To inspect soil and bituminous testing laboratories, distribute proficiency test samples, and support the voluntary standards development process.

\section{Problem}

The quality of testing in construction materials laboratories is an important concern when considering the overall question of quality of construction. The importance of the testing function is demonstrated by The Strategic Highway Research Program (SHRP) which was initiated in 1987 as a 5 year, $\$ 150$-million highway and bridge research program. Standardization of testing to enhance the reliability of quality assurance measurements is of paramount concern. The productivity of the testing community can be increased by the use of correct procedures and apparatus which reduce testing errors and provide a sound basis for the acceptance of materials on certificate. More efficient use of longestablished construction materials and broader use of new materials are facilitated by dependable quality assurance programs.

\section{Approach}

With the support of AASHTO Research Associates working under NIST supervision, services are provided to both public and private laboratories on a voluntary basis. These services include the on-site inspection of the laboratory and the distribution of proficiency test samples. The current scope of the laboratory inspection program (LIP) includes the testing of soils, bituminous materials, and plastic pipe, and the measurement of roughness and frictional properties of highways. Equipment and procedures used in performing conventional quality assurance tests are evaluated for conformance to applicable national standards. Proficiency test samples of asphalt, soils, paint, aggregates and bituminous concrete are distributed at regular intervals. Additionally, technical studies are conducted in areas related to these programs, often in conjunction with other NIST units.

Specific products of this work include: 1 . detailed inspection reports; 2 . report on each round of proficiency sample testing; 3 . input to the work of standards committees such as draft standards and precision data; and 4. reports on the results of technical studies. The AMRL programs provide the following benefits to construction materials testing laboratories and others involved with the nation's transportation systems: 1 . improves the quality of laboratory testing; 2 . provides data to quantify standard measurement techniques; and 3. provides direct communications between testing laboratories and standards-writing committees.

\section{Recent Results}

During FY 1993, over 180 bituminous and soil laboratories were inspected, over 4000 proficiency samples were distributed, and corresponding test reports were issued.

Spellerberg, P., Trimm, W., and Pielert, J., "Development and Application of a Quality System Standard for Construction Materials Testing Laboratory," Proceedings of ASTM E36 Symposium on Operation and Accreditation of Laboratories (submitted). 


\section{Construction of Addition to CMRL Proficiency Sample Building}

\author{
Principal Investigator: \\ James $\mathrm{H}$. Pielert \\ Building Materials Division \\ 301-975-6704
}

\section{Sponsor:}

American Society for Testing and Materials (ASTM)

American Association of State Highway and

Transportation Officials

\section{Objective}

To expand BFRL's Construction Materials Research Laboratory (CMRL) Proficiency Sample Facility (Building 206) to accommodate the increasing laboratory participation in the Proficiency Sample Programs of CCRL and AMRL.

\section{Problem}

Laboratory participation in the CCRL and AMRL Proficiency Sample Programs has increased significantly in recent years to a current level of almost 800 laboratories who receive 7000 samples annualIy. This increase is a reflection of the importance of the programs in promoting the quality of testing of construction materials in the United States. Building 206 where the proficiency samples are prepared is fully utilized in meeting current commitments leaving little room for growth in existing programs or the addition of new programs.

\section{Approach}

Construct a $175 \mathrm{~m}^{2}$ addition to NIST's Building 206. The BFRL Proficiency Sample Facility Construction began early in FY 1993 and was completed in May 1994.

Recent Results

Facility dedication, May 1994.

\section{Durability of Polymer/Glass Fiber Composites for Building Applica- tions}

\author{
Principal Investigator: \\ Tinh Nguyen \\ Building Materials Division \\ 301-975-6718
}

Sponsor:

National Institute of Standards and Technology

Objective

To develop a technique for assessing the durability of polymer/glass fiber composites.

\section{Problem}

There is a growing interest in the use of composites for building and construction. For these applications, the effects of the environment on the mechanical properties of the materials is a prime concern. The loss of bond strength between the fiber and the matrix in the presence of liquid water or high relative humidities has been recognized as the most important factor reducing the service life of a polymer/glass fiber composite. Also, extensive data in the literature on durability of adhesives and coatings on metals show that water at the poly$\mathrm{mer} / \mathrm{substrate}$ interface is the main cause of the loss of adhesion of these materials when exposed to water or high relative humidities.

Although polymer/glass fiber composites have been in use for many years in aerospace applications, there is still no simple method available for evaluating their bond strength in the presence of water or high relative humidities. In the past year, we have, for the first time, developed a method, based on the theory of FTIR-multiple internal reflection (FTIR-MIR) spectroscopy, to measure the thickness of the water layer at the interface between a polymer film and a silica substrate. We observed that, as water accumulates at the polymer/substrate interface, the system loses bond strength. Based on the literature data and our own observations, we believe that there should be a strong correlation between the thickness (or amount) of the water layer at the polymer/substrate interface and the loss of bond strength in a glass/fiber composite. If such a correlation exists, FTIR-MIR spectroscopy (combined with the equations we have developed) would be a 
powerful technique for nondestructively assessing the durability and the service life of glass/fiber composites. This technique should be particularly useful for screening new composites. The uniqueness of this technique is based on the facts that FTIR spectroscopy is very sensitive (nanogram) to water, its dissociated groups, and its state of bonding; it can be used at ambient conditions and, when combined with internal reflection, it detects water in the polymer/substrate interfacial region without interference from water vapor in the environment.

\section{Approach}

During FY 1994, BFRL will develop data that relate the thickness of the water layer at the interface with the bond strength level of a polymer/glass composite. Epoxy resin will be applied to silanetreated and untreated $\mathrm{SiO}_{2}$-covered $\mathrm{Si}$, and silane treated flat $\mathrm{E}$ glass substrates are used for the study. The thickness of the water at the epoxy/glass interface is determined using Fourier transform infrared-multiple reflection (FTIR-MIR) spectroscopy and the bond strengths of the epoxy/glass systems (flat plates and fiber composites) in the presence of water are measured by peel and shear tests. Experimentally, a clear epoxy resin is applied to silane-treated and untreated $\mathrm{SiO}_{2}$-covered Si internal reflection elements, which serve as the "glass" substrates. A water chamber is attached to the epoxy coated substrate. After adding water to the chamber, FTIR-MIR spectra are taken automatically at specified time intervals. Using BFRL's models, the thickness and amount of the water layer at the epoxy/substrate (silane treated and untreated) interface will be determined. The peel strengths, as a function of water exposure, of epoxy on silane-treated and untreated $\mathrm{SiO}_{2}$-covered Si wafers and silane-treated flat $\mathrm{E}$ glass plates are measured using a wet adhesion peel test apparatus developed by BFRL. The shear strength of epoxy/silane treated $\mathrm{E}$ glass fiber composites as a function of time exposed to water is determined using interlaminar shear strength (ILSS) composite specimens. The thickness of the water layer at the epoxy/substrate interface, the bond strengths of flat panels and of the composites as a function of exposure, and the rate of water transport through the epoxy on the substrate, are analyzed and correlated.

The results of this study are expected to provide the data to assess the applicability of a spectroscopic technique of nondestructively testing the durability of glass/polymer composites used in humid environments.

Recent Results

New project. 


\section{Performance Criteria for Single-Ply Roofing Membranes}

Principal Investigator:

Walter J. Rossiter, Jr.

Building Materials Division

301-975-6719

\section{Sponsor:}

National Institute of Standards and Technology

\section{Objective}

To develop performance criteria for single-ply roofing membranes that assist in the selection, evaluation, and maintenance of single-ply membrane materials.

\section{Problem}

Single-ply roofing membrane materials account for about two-thirds of the low-sloped membrane systems installed annually in the United States. Ease of installation, performance, cost, and architectural considerations have influenced the rapid acceptance of the materials. Nevertheless, survey information from the National Roofing Contractors Association (NRCA) indicates more performance problems with single-ply systems than with built-up systems. Of the problems reported, defective laps and seams have been the most frequent, accounting for about 24 percent of those reported. The 1987 NIST/NRCA Round Table on Roofing Research stated that the roofing industry "lacks a significant data base of field performance on which service life may be predicted," and that "the factors affecting roofing performance must be more fully understood to assure success with new materials and systems."

in 1989, BFRL published Building Science Series 167, Interim Criteria for Polymer-Modified Bituminous Roofing Membrane Materials. While being a significant step towards the development of criteria for modified bituminous membranes, the report did not suggest criteria and test methods for some of the identified performance requirements. Two notable examples are seam performance and weathering resistance. Data from the field and laboratory are needed to support development of the needed criteria.

\section{Approach}

During FY 94, BFRL will continue to provide technical assistance to ASTM Committee D08 on Roofing,
Waterproofing, and Bituminous Materials, and to the RILEM/CIB Committee on Membrane Roofing Systems. Both committees are developing standards or prestandards for single-ply and polymermodified bitumen roofing membranes. BFRL will collaborate with the National Roofing Contractors Association in organizing their 1995 NISTBFRL/NRCA Roofing Conference. The NISTBFRL/NRCA roofing conferences are the nation's main forum for disseminating results of roofing research. BFRL's leadership role in standards committees helps assure the development of sound standards for the U.S. roofing industry.

\section{Recent Results}

Rossiter, W.J. and Denchfield, R.D., "A Field Study of the Performance of Polymer-Modified Bitumen Roofing," 10th NIST/NRCA Conference on Roofing Technology, National Roofing Contractors Association, Rosemont, IL, pp 48-58, April 1993.

Gaddy, G.D., Cullen, W.C., Barbari, T.A., and Rossiter, W.J., "Impact of Exposure Conditions on the Mechanical Properties of Polyisocyanurate Foam Insulations," 10th NIST/NRCA Conference on Roofing Technology, National Roofing Contractors Association, Rosemont, IL, pp 64-71, April 1993.

Rossiter, W.J., Martin, J.W., Embree, E., Seiler, J.F., and Byrd, W.E., "The Effect of Ozone on the Creep-Rupture of Butyl-Adhered EPDM Seam Specimens," 10th NIST/NRCA Conference on Roofing Technology, Roofing Contractors Association, Rosemont, IL, pp 85-92, April 1993. 


\section{Serviceability of Roofing Systems}

Principal Investigator:

Walter J. Rossiter, Jr.

Building Materials Division

301-975-6719

\section{Sponsor:}

Department of the Army

Construction Engineering Research Laboratory

\section{Objective}

To assist the U.S. Army develop their technical basis for selecting and installing serviceable lowsloped roofing systems.

\section{Problem}

For over a decade, the U.S. Army has investigated the field performance of single-ply roofing systems as alternatives to bituminous built-up roofing (BUR) systems. Army Guide Specifications were developed for the single-ply systems on the basis of the information obtained from the field investigations. However, these Guide Specifications are limited to the laboratory evaluation of the serviceability of the new membrane materials. In particular, the test methods used are not performance oriented, but differ depending on the type of membrane material. This is a restrictive approach that makes comparison of test results from different materials difficult, and hampers decisions on selecting appropriate systems for particular installations. Consequently, the Construction Engineering Research Laboratory (CERL) has initiated a study to develop evaluative laboratory procedures that are equally applicable to the different types of single-ply membrane materials. A leading candidate is the use of change in a material's strain energy during exposure.

\section{Approach}

During FY 1994, BFRL will perform this work in two phases. In Phase 1, a study will be conducted to investigate the use of instrumental methods of analysis for determining changes that may occur in roof membrane materials due to heat aging. The analytical methods will include: Fourier Transform Infrared (FTIR) Spectroscopy, Secondary Ion Mass Spectroscopy (SIMS), Differential Scanning Calorimetry (DSC), Thermogravimetry (TG) Analysis, and Nuclear Magnetic Resonance (NMR). Samples of new (unaged) and heat-aged roof membrane materials will be supplied by CERL, and be repre- sentative of the newer products available on the U.S. market over the last decade (i.e., EPDM, PVC, APP-modified bitumen, and SBS-modified bitumen). For purposes of comparison, conventional built-up bituminous samples will be included as a control. A key aspect of the study will be to determine whether any of the analytical methods are applicable to the characterization of all the generic types of membrane materials. In Phase 2, laboratory testing and data analysis will be conducted by CERL and BFRL. The details will be decided upon in discussions with CERL.

This work will advance test methods that are suitable for use in performance criteria for single-ply membrane materials.

Recent Results

New project. 


\section{Materials and Processes for Durable Relocatable Rigid Wall Structures}

Principal Investigator:

James R. Clifton

Building Materials Division

301-975-6707

Sponsor:

Department of the Army

NATICK Research, Development, and Engineering

Center

\section{Objective}

To provide scientific and technical support to the DoD Joint Committee on Tactical Shelters in the development of new material and processing standards and the updating and revision of existing standards.

\section{Problem}

DoD requires standards for materials and processes for durable rigid wall relocatable structures to ensure that they give the desired performance. In addition to strength, durability, and environmental requirements, new requirements are being imposed by changes in military threat scenarios. DoD encourages the use of consensus standards and with changing military requirements, many existing standards need to be revised and new standards developed.

\section{Approach}

During FY 1994, BFRL will continue providing scientific, technical, and administrative leadership in the generation of new standards and the revision of existing standards through ASTM Subcommittee E06.53, "Materials and Processes for Durable Rigid Wall Relocatable Structures." Subcommittee meetings will be organized and conducted and coordination provided between the subcommittee and ASTM Committee E06. The requests of the DOD Joint Committee on Tactical Shelters will also be addressed in the development and revision of standards.

BFRL has chaired the Committee for two decades. The Committee's accomplishments under BFRL leadership include:
- $\quad$ E866 "Standard Specification for CorrosionInhibiting Adhesive Primer for Aluminum Alloys to be Adhesively Bonded in Honeycomb Shelter Panels."

- $\quad$ E874 "Standard Practice for Adhesive Bonding of Aluminum Facings to Nonmetallic Honeycomb Core for Shelter Panels."

- New Draft Standard, "Standard Specification for Rigid Foam for Use in Shelter Sandwich Panel Cores."

- New Draft Standard, "Standard Specification for Epoxy Resin System for Composite Skin, Honeycomb Sandwich Panel Repair."

- New Draft Standard, "Standard Specification for Structural Paste Adhesive for Sandwich Panel Repair."

Documentation of accomplishments provide a benchmark for evaluating the effectiveness of the Committee.

Recent Results

Three meetings of ASTM Subcommittee E06.53 were organized and conducted. Two standards were revised (E866 and E874) and approved by ASTM and three new standards were submitted to Main Committee E06 ballot. Quarterly progress reports were submitted to DoD. 
BUILDING ENVIRONMENT DIVISION

\section{THERMAL MACHINERY}




\section{Thermodynamic Performance of Natural Fluids as Alternative Refrigerants}

\author{
Principal Investigator: \\ David A. Didion \\ Building Environment Division \\ 301-975-5881
}

\section{Sponsor:}

National Institute of Standards and Technology

\section{Objective}

To evaluate the thermodynamic and heat transfer performance of natural fluids as a working medium for refrigeration systems.

\section{Problem}

The refrigerant industry is rapidly using hydrofluorocarbon compounds as a method to eliminate chlorine in the manufacture of refrigerants. This conversion has and continues to be a dramatic change to this industry. However, on the horizon looms the global warming crisis and the carbonfluorine bond of the alternative refrigerants. Since the atmospheric chemical kinetics of man-made materials are complex and difficult to predict it seems that such fluid offers the potential for trouble for future generations and solutions may lie in the using "natural" fluids as refrigerants. Such fluids are found in nature or are in abundance in the atmosphere already that the marginal difference the refrigerant supply would make would be insignificant (e.g., carbon dioxide, hydrocarbons, ammonia, water vapor). Although these fluids have been used as refrigerants in the past, they (except for ammonia in industrial applications) were disregarded for various safety, efficiency, or economic reasons. These fluids need to be reanalyzed to determine if the above problems can be alleviated through modern technology and alternative cycle design.

\section{Approach}

During FY 1994, BFRL will determine the optimum vapor compression/absorption cycle for several of fluids and their mixtures. This research will be performed with NIST's Chemical Science and Technology Laboratory. Work will require modifying BFRL's basic CYCLE-11 family of models. Optimization will focus on efficiency. Criteria for acceptance to the laboratory evaluation stage will be the same as traditional refrigerants; i.e., safety, oil compatibility, specific capacity, pressure ratio, maximum! temperature, etc.

The difficulties and economics associated with development of a high pressure positive displacement compressor and a low pressure centrifugal compressor will be explored. BFRL's heat pump simulation model, HPSIM, will be upgraded to use REFPROP 4 property routines for thermodynamic and transport property evaluation. A property lookup table will be employed to address most computational-intensive property ranges, while the REFPROP routines will be called directly for properties which are outside the table range. This lcok-up-table approach is needed to mitigate significant CPU requirements, which result from complexity of the model itself and the REFPROP routines. The model will be able to evaluate performance of an air-to-air heat pump charge with a mixture comprising up to five components. Several modifications will be made to better represent newer heat transfer surfaces introduced to the market place in the recent years.

A thermodynamic model study, using NIST's Cycle 11 , is being conducted for zerotropic mixtures of the vapor pressure range that is currently used by refrigeration machinery. This effort will include flammable fluids and must necessarily incorporate within the hardware system the ability to isolate the flammable fluid from the building interior. It is intended to design such a system and evaluate the relative performance (i.e., compare to existing system efficiencies) to determine if such a concept is acceptable.

\section{Recent Results}

Didion, David A., "A Simulation Model and Study of Hydrocarbon Mixtures for Residential Heat Pump Systems," IIR Conference on Natural Working Fluids, Hannover, Germany, May 1994 (to be published). 


\section{Thermodynamic Performance of Alternative Refrigerants and Refrigerant Mixtures}

\author{
Principal Investigator: \\ David A. Didion \\ Building Environment Division \\ 301-975-5881 \\ Peter Rothfleish \\ Building Environment Division \\ 301-975-5868
}

\section{Sponsor:}

National Institute of Standards and Technology

Department of Energy

Office of Building Technology

Building Equipment Division

\section{Objective}

To quantify the practical limitations and performance benefits of using a zeotropic refrigerant mixture in a residential air-to-air heat pump.

\section{Problem}

The use of zeotropic mixtures as working fluids for refrigeration machines offers two basic advantages: 1. a gliding saturation temperature during the phase change of the working fluid (i.e., evaporation and condensation) and 2 . an opportunity to vary the circulating working fluid's composition and, thus, the system's volumetric capacity. The indoor heat exchanger's temperature glide offers the opportunity to improve the system's coefficient of performance (COP) if counterflow between the air and refrigerant can be established. The degree to which this ideal temperature profile matching can be achieved depends very much on the design of the heat exchanger, and the ideality of the mixture. Thus, practical trade-offs with other criteria demands on the hardware and fluid selection result in a certain degree of subjective judgement, based on experience, to be exercised in the design of an optimized system. The problem is to acquire sufficient data on the components to establish their ideal performance potential. This data will be used to develop the "optimum" performance design of a zeotropic heat pump, which will be modeled, designed, constructed, and evaluated in the laboratory.
Work on the counter-flow and cross-counter flow heat exchangers was conducted in BFRL laboratories during the recent past. A composition-shifting study was conducted prior to FY 1993 using a passive system (i.e., the accumulator) which yielded limited results. During FY 1993 an active distillation column was designed and constructed. This design has sufficient uniqueness that a patent application is being considered. Major modifications were made to the compressor system enabling the input power to be measured directly at the compressor (rather than at the motor).

\section{Approach}

During FY 1994, BFRL will evaluate the composition shifting capabilities of its designed and constructed active distillation column and evaluate its impact on the heating performance of the heat pump. The inequities of alternative refrigerants proposed by industry are zeotropic mixtures. These mixtures have the capability of shifting their composition if the machinery is designed to allow for it. This study will provide measure of the potential for increasing these mixture's refrigeration capacity and thus minimize the resistance heat requirement for residential heat pumps.

The need to predict, a priori, the composition shift under uncontrolled conditions (e.g., slow leak) has stimulated the development of a computer simulation based on REFPROP. This new program, known as LEAK, is being developed and verified by experiment for the purposes of determining if a flammable condition may occur. Current ASHRAE requirements require these predictions for all mixtures applying for an official rating. The intent of this development is to provide the industry with this prediction capability to minimize their testing requirements.

Recent Results

Rothfleish, P. and Didion, D., A Study of Heat Pump Performance Using Mixtures of R32/R134a and R32/R125/R134a as 'Drop-In' Working Fluids for R22 With and Without a Liquid-Suction Heat Exchanger, NISTIR 5321, National Institute of Standards and Technology, Gaithersburg, MD, December 1993.

Domanski, P. and Didion, D., "Thermodynamic Evaluation of R-22 Alternative Refrigerants and Refrigerant Mixtures," ASHRAE Transactions, v99, 2, pp 636-648, June 1993. 
Kim, M., Mulroy, W., and Didion, D., "An Experimental Evaluation of the Flammability and Performance Potentials of Two Azeotropic Refrigerant Mixtures," 6th Int'\% Symposium on Transport Phenomena in Thermal Eng. (ISTP-6), May 1993.

Domanski, P., Mulroy, W., and Didion, D., "Glide Matching with Binary and Ternary Zeotropic Refrigerant Mixtures," Part 1: An Experimental Study; Part 2: A Computer Simulation, International Conference in Energy Efficiency in Refrigerant and Global Warming Impact, Univ. of Ghent, Belgium, May 12-14, 1993.

Domanski, P. and Didion, "Theoretical Evaluation of R22 and R502 Alternatives," Final Report to ARTI, ARTI MCLR Project Number 65050900(DOE/CE/23810-7), January 1993.

\section{Calorimetric and Visual Study of Boiling Enhancements with Refrig- erant 134a and Horizontal Two- Phase Flow of R-32/134a}

\author{
Principal Investigator: \\ Mark A. Kedzierski \\ Building Environment Division \\ 301-975-5282 \\ David A. Didion \\ Building Environment Division \\ 301-975-5881
}

\section{Sponsor:}

National Institute of Standards and Technology

Department of Energy

Office of Building Technologies

Building Equipment Division

Electric Power Research Institute

Customer Systems Division

\section{Objective}

To generate heat-transfer data and visual observations for a thorough description of the boiling behavior of the refrigerant $R-134 a$ with various lubricants and heat transfer additives and to measure the flow boiling heat transfer coefficient for a new R-22 replacement: a 30\% mass R-32/70\% mass R-134a/ mixture.

\section{Problem}

The introduction of alternative refrigerants has solved one problem (ozone destruction in the stratosphere by chlorine) and created another (lack of thermal design information for the new refrigerants). Within the next few years, most new refrigeration equipment will be required by law to operate with ozone-safe refrigerants. Industry does not have an ozone-safe, drop-in refrigerant for their equipment so they must redesign equipment. This is an industry concern since they have little experience designing chillers for alternative refrigerants. The refrigeration industry requires accurate twophase heat transfer correlations to redesigned evaporators and condensers to ensure the efficient and reliable operation of centrifugal water chillers and direct expansion evaporators. 


\section{Approach}

During FY 1994, BFRL will advance alternative heat transfer research for the direct-expansion and flooded evaporator industries. The tube-side flow boiling study of the R-32/134a/lubricant mixture will be conducted in an existing test apparatus and will aid the direct-expansion heat exchanger industry. The R-134a/additive/lubricant study will be conducted in a pool boiling calorimetric/visualization rig and will assist the flooded evaporator industry.

Heat-transfer data and visual observations will be generated for a thorough description of the boiling behavior of three commercial enhanced surfaces with several combinations of alternative refrigerant, lubricant, and additive. Two refrigerants will be examined: R-134a and R-12 (as a baseline for comparison to R-134a). R-134a will be tested with two lubricants and one additive having a potential to enhance the pool-boiling performance. The additives have not been previously tested. Finally, mixtures of the alternative refrigerants with the lubricants and additives will be tested. In total, 21 tests will be performed for one particular lubricant and additive concentration. Each additional lubricant and additive concentration involve 30 tests. Each test includes many individual data points for various conditions. Consequently, the work will produce a substantial, encompassing data source for use by the refrigeration industry.

The R-32/134a mixture study will investigate horizontal flow boiling of a $30 \%$ mass R-32/70\% mass R-134a mixture, a R-32/125/134a mixture, and $\mathrm{R}-22$ with lubricants. The mixtures have been chosen for study by the R-22 Alternative Refrigerants Evaluation Program (AREP). This program was organized by the Air-Conditioning and Refrigeration Institute "to investigate and evaluate alternative refrigerants to replace HCFC-22 in major air-conditioning and refrigeration applications." This investigation is expected to generate valuable heat transfer information for the design of new R-32/134a evaporators and condensers.

The results of this research are expected to establish a database to serve as a technical resource to design efficient refrigeration equipment. Efficiency improvements can potentially result from special lubricants or heat transfer additives.
Recent Results

Kedzierski, M. A. and Kaul, M. P., "Horizontal Nucleate Flow Boiling Heat Transfer Coefficient Measurements and Visual Observations for R-12, R134a, and R-134a/Ester Lubricant Mixtures," 6th International Symposium on Transport Phenomena, Seoul, May 9-13, 1993.

Kedzierski, M. A., Simultaneous Visual and Calorimetric Measurements of R-11, R-123, and R123/Alkylbenzene Nucleate Flow Boiling, NISTIR $92-$ 4948, National Institute of Standards and Technology, Gaithersburg, MD, 1992

Kedzierski, M. A., Kim, J. H., and Didion, D. A., "Causes of the Apparent Heat Transfer Degradation for Refrigerant Mixtures," HTD-Vol. 197, TwoPhase Flow and Heat Transfer, ASME, pp 149-158, 1992. 


\section{Mixtures of Fluorocarbons as Alternatives for R11, R12, R22, and $\mathrm{R} 502$}

Principal Investigator:

William J. Mulroy

Building Environment Division

301-975-5878

David A. Didion

Building Environment Division

301-975-5881

\section{Sponsor:}

Electric Power Research Institute

Customer Services Division

\section{Objective}

To determine whether there exist environmentally acceptable binary and ternary mixtures of partially halogenated fluorocarbons that act as an azeotrope or near-azeotrope alternative for R11, R12, R22, and $\mathrm{R} 502$.

\section{Problem}

Research performed during the past four years has nearly exhausted pure fluid candidates as potential alternatives for new systems. These efforts have not produced drop-in candidates for existing systems. The search for alternatives among pure materials has limited the search for alternative possessing the desired attributes. The number of possible alternatives is significantly expanded when mixtures are considered. With mixing different fluids, there is the option of tailoring a material by modifying composition, an option not available with a pure material. Already, some mixtures have been identified as drop-in candidates for existing systems.

\section{Approach}

During FY 1994, BFRL will use its recently developed method to predict azeotropes and the degree of near azeotropy, that a given set of components may demonstrate. Once candidate mixtures are identified, based on relevant thermodynamic properties, their thermodynamic performance will be predicted using different versions of NIST cycle model, CYCLE-11. Ideal and nonideal cycle analysis will be used. In addition, the benefit of liquidsuction line heat exchange will be quantified for those candidates whose heat capacity appears favorable. The expected benefit of this research will be increased confidence that the best alternative refrigerants to those to be phased out under the Montreal Protocol have been identified.

Recent Results

New project. 


\section{Minimizing Throttling Losses in the Refrigeration Cycle}

Principal Investigator:

Piotr A. Domanski

Building Environment Division

301-975-5877

David A. Didion

Building Environment Division

301-975-5881

\section{Sponsor:}

National Institute of Standards and Technology

Advanced Technology Program (ATP)

\section{Objective}

To identify the best application for the ejector cycle and rate it against the economizer cycle and the cycle with liquid-line/suction-line heat exchange.

\section{Problem}

Due to large molecular structure and heat capacity, alternative non-CFC refrigerants have substantial throttling losses when used in a basic reversed Rankine cycle. These losses degrade their cycle efficiency below that of the original CFC fluids. An ejector, economizer, and liquid-line/suction-line heat exchanger minimize throttling loses and improve cycle efficiency. The theoretical merits of these three addenda to the refrigeration cycle will be evaluated and the best application for the ejector cycle will be indicated. This study will be performed in support to the ATP effort on Ejector Expansion Refrigeration Cycle by Calmac Manufacturing Company.

\section{Approach}

During FY 1994, BFRL will identify the thermodynamic properties of the refrigerant that are essential for improving the performance of the ejectorexpansion cycle. This task will require a theoretical analysis reduced to the basic refrigerant properties. In the modeling part of the study, BFRL researchers will simulate the performance of the ejector cycle, economizer cycle, and IIsI-hx cycle. Theoretical models will be developed for these cycles based on the CYCLE-11 model developed at BFRL. Simulations will be performed for different applications and different fluids. Work will be performed jointly with NIST's Chemical Science and Engineering Laboratory to obtain the thermophysical properties of the refrigerants used. The study will identify the most promising applications for the ejector cycle application meaning both operating regimes used working fluids. Useful information is expected to be derived from theoretical netting of the ejector cycle, economizer cycle, and the cycle with liquid-line/suctionline heat exchange since they provide difficult efficiency improvement, but are also vastly different in design and ccst.

Recent Results New project. 
BUILDING ENVIRONMENT DIVISION

\section{MECHANICAL SYSTEMS AND CONTROLS}




\section{Communication Protocols for Building Controls}

\author{
Principal Investigator: \\ Steven T. Bushby \\ Building Environment Division \\ 301-975-5873
}

\section{Sponsor:}

National Institute of Standards and Technology and

\section{Department of Energy}

Office of Building Technologies

Federal Energy Management Program

\section{Objective}

To assist the building industry in the development, evaluation, and conformance testing of Communication Protocol Standards for the open exchange of information between equipment from different control vendors and between different levels of control in both hierarchal and distributed building management systems (BMS).

\section{Problem}

Today's direct digital control (DDC) systems employ proprietary communication protocols which prevent systems supplied by different manufacturers from communicating with each other. This has resulted in "captive customers" who, upon buying a control system, are unable to upgrade or expand it without going back to the same manufacturer. This lack of communication capability between control systems made by different manufacturers also prevents the building owner from obtaining the most capable building service by not allowing him to choose, regardless of the manufacturer, the best energy management system, the best digital controllers, the best security system, the best fire detection system, or the best telecommunications system.

\section{Approach}

During FY 1994, BFRL will:

1. Revise/Refine BACnet standard as a result of public review process.

2. Refine BACnet Reference Implementation to reflect changes in the draft standard.

3. Administer and participate with industry partners in a BACnet Interoperability Testing consortium.

The consortium will: a. assist control system manufacturers verify the correctness and interoperability of their proprietary BACnet implementations;

b. verify the technical soundness of the BACnet protocol;

c. identify errors or omissions in the BACnet protocol specification before it becomes a final standard;

d. identify ambiguities in the BACnet protocol which might lead to implementations which cannot interoperate and;

e. develop insight into testing requirements which should be included in a future ASHRAE standard for testing conformance to BACnet.

4. Develop Conformance Test System - a combination of hardware and software that can execute tests on vendor's products and evaluate the results of each test (pass, fail, inconclusive) automatically or semiautomatically.

5. Begin to create a Conformance and Interoperability Testing Program in conjunction with industry, ASHRAE, and other government agencies. 6. Begin to expand BACnet to include integrated building services, including life safety, security, and transportation.

The expected benefits of this work are competitive procurement of building control system products, more efficient and lower cost operation of commercial buildings, expanded markets for DDC technology, and safer, more comfortable working environments for building occupants.

\section{Recent Results}

Developed a draft ASHRAE Standard 135P working with ASHRAE's Standards Project Committee 135P.

Bushby, S.T., "BACnet Promises a New Age in Building Control Systems," Construction Business Review, vol. 4, no. 2, pp 62-67, March/April 1994.

Bushby, S.T. and Newman, H.M., "BACnet: A Technical Update," ASHRAE Journal, vol. 36, no. 1, pp S72-S84, January 1994. 


\section{Real Time Fault Detection and Diagnostics}

\author{
Principal Investigator: \\ George E. Kelly \\ Building Environment Division \\ 301-975-5870
}

Sponsor:

National Institute of Standards and Technology

Department of Energy

Office of Building Technologies

Building Systems Division

\section{Objective}

To develop methods for performing fault detection and diagnostics on mechanical equipment and systems in real time and participate in the International Energy Agency (IEA) Annex 25 Committee on Real Time Simulation of HVAC - Systems For Building Optimization, Fault Detection and Diagnostics (BOFD).

\section{Problem}

The operation of buildings and building systems is a complex procedure. With more and more emphasis on a combination of different and often conflicting performance measures, the processes, systems, and equipment used in commercial and residential buildings are becoming more complex. New control and on-line analysis methods are needed to detect problems (faults) as they occur, determine which component or system is failing or has failed, and recommend maintenance or repair procedures. These methods then can be used by the building controls industry into building energy management system (BEMS), "smart" building equipment, and into stand alone systems dedicated to fault detection and diagnostics.

\section{Approach}

During FY 1994, BFRL and Johnson Controls, Inc. through a Cooperative Research and Development Agreement (CRADA) will use BFRL's Fault Detection and Diagnostics (FDD) Laboratory containing a variable air volume (VAV) air handling system to study problem associated with control strategies and algorithm commonly used today on VAV air handling systems. Experimental data will be gathered on the performance of various components and control algorithms under normal operating conditions and without/with the presence of typical faults. A detailed $\mathrm{HVACSIM}^{+}$based model of the VAV air handling system will be developed and validated, and simulation studies will be performed to evaluate the performance of alternative control techniques and strategies and various fault detection/diagnostic methods.

These technologies will be implemented and tested on an actual VAV air handling system in the laboratory. Various methods for on-line system identification, fault detection, and diagnosis will be explored in conjunction with the new control methods and used to support BFRL and DOE participation in the International Energy Agency Annex 25.

This work is expected to improve the operation and performance of complex building systems, promote energy conservation, and help create a market for intelligent building management systems that should have a significant positive impact on both domestic and international sale of "smart" controls.

\section{Recent Results}

Kelly, G.E. et.al., "Using Emulators to Evaluate the Performance of Building Energy Management Systems", ASHRAE Transaction, New Orleans, January 1994.

Peitzman, H., Park, C. et.al., "The Reproducibility of Tests on Energy Management and Control Systems Using Building Emulators," ASHRAE Transaction, New Orleans, January 1994.

Kelly, G.E. et.al., Guidelines for Using Emulators to Evaluate the Performance of Energy Management and Control Systems, NISTIR 4991, National Institute of Standards and Technology, Gaithersburg, MD, 1992. 


\section{Test Procedures for Furnaces, Boilers and Integrated Appliances}

\author{
Principal Investigator: \\ Stanley T. Liu \\ Building Environment Division \\ 301-975-5880
}

\section{Sponsor:}

Department of Energy

Office of Building Technologies

Codes and Standards Division

\section{Objective}

To provide equitable testing and rating procedures for determining energy performance of furnaces, boilers, and integrated appliances.

\section{Problem}

The ANSI/ASHRAE Standard 124-1991, Method of Testing for Rating Combination Space Heating/Water Heating Appliances, has been approved by the American National Standard Institute and ASHRAE. The Standard requires the testing of the space heating function of the appliance as a boiler in accordance with the ANSI/ASHRAE Standard 1031988 , and testing the water heating function as a water heater in accordance with a procedure similar to the DoE test procedure for water heaters. Rating descriptors for an appliance are derived from the two separate tests. In FY 1993, BFRL conducted a computer simulation study on a family series of five boilers with an identical tankless coil for domestic hot water in accordance with the ASHRAE Standard 124 test procedure for developing an interpolation scheme that would reduce the number of tests required for a family of boilers of similar construction. The result revealed that a liriear interpolating and/or extrapolating scheme of the rating descriptors based on test data from two boilers in a family series can be used to determine the performance of the other boilers. BFRL also contracted with the ETL testing laboratory to conduct laboratory tests on a family of oil-fired boilers with tankless coil for domestic hot water production and conducted laboratory tests at BFRL on a family of gas-fired boilers with a storage tank for domestic hot water.

In FY 1991, BFRL developed and delivered to DoE a recommended draft test procedure for furnaces and boilers that references ANSI/ASHRAE Standard 103-1988, and revisions to the test procedures for vented home heaters and pool heaters. The draft procedures also establish a new annual energy efficiency rating descriptor which included the energy consumption of the auxiliary electrical components of the appliances in determine their overall energy efficiency. The new procedures were evaluated by DOE during FY 1992-1993, and were approved for publication in the Federal Register by DoE as a Propused Rule Making in August 23, 1993 for public hearing and comments. A detailed evaluation of the comments on the new procedures received from industries and other interested organizations during the comment period will need to be carried out and revisions made. BFRL will evaluate the comments and suggestions, make the appropriate revisions, and assist DOE complete the test procedures before it is published by DOE as a Final Rule.

\section{Approach}

During FY 1994, BFRL will perform the following tasks:

1. Review all comments received on the Proposed Rule Making for furnaces/boilers and vented home heaters and assist DOE publish the Final Rule for these products.

2. Revise the computer program AFUEBF by incorporating the procedures that are in the final rule for distribution to industry.

3. Complete laboratory tests on the performance of a family of boilers in combination with tankless coils and storage tanks to validate the linear interpolating methodology.

4. Evaluate and modify, if necessary, the first hour draw test for Type I combination appliance (as a water heater) as specified in ASHRAE 124 for boiler with tankless coils.

5. Develop a recommended test procedure for evaluating the annual performance and cost of operation of Type I and Type II combined space/water heating appliances and assist DoE in preparing a Proposed Rule Making on this product. 6. Assist DOE prepare responses to Requests for Waivers and draft Federal Register Notices of proposed Rule Making.

The development of the test procedures is expected to provide a uniform method of test on the energy performance of furnaces, boilers, and integrated appliances so that similar appliances can be rated equally and fairly and their compliance with the minimum energy standards determined. The 
development of a linear interpolating method will reduce the number of rating tests and the burden that the manufacturers of integrated appliances have to conduct for a family series of models of a similar construction.

\section{Recent Results}

Liu, S.T. and Kelly, G.E., Predicting the Energy Performance Ratings of a Family of Type I Combination Appliances, NISTIR 5250, National Institute of Standards and Technology, Gaithersburg, MD, August 1993.

\section{Test Procedures for Heat Pumps and Air Conditioners}

\author{
Principal Investigator: \\ Brian Dougherty \\ Building Environment Division \\ 301-975-6396
}

\author{
Sponsor: \\ Department of Energy \\ Office of Energy Efficiency and Renewable Energy \\ Office of Codes and Standards \\ Appliance Division
}

\section{Objective}

To provide equitable testing and rating procedures for determining energy performance of heat pumps and air conditioners.

\section{Problem}

The Energy Policy and Conservation Act (PL 94163) (EPCA), as amended, requires the Department of Energy (DOE) to prescribe test and rating procedures and minimum performance standards for various residential and commercial appliances. DoE has, since 1975, relied on BFRL to develop and then update the test and rating procedures.

\section{Approach \\ During FY 1994, BFRL will:}

1. Edit the DoE test procedure for residential heat pumps and air conditioners. Much of the document will be reorganized. The nomenclature will be revised to be consistent with the nomenclature within the forthcoming DoE proposed test procedure for combined heat pump - water heating appliances. A few new issues may also be addressed, including how to test units having variable-speed, constant air flow rate indoor fans.

2. Assist with finalizing the DoE test procedure and the Air-Conditioning and Refrigeration Institute (ARI) rating procedure for combined heat pump - water heating appliances. BFRL will also participate in resolving comments from the first public review of the proposed combined appliance test method standard 137P of the American Society of Heating, Refrigerating, and Air-Conditioning Engineers (ASHRAE).

3. Initiate work to substantially revise the DoE test procedure for heat pumps and air conditioners. BFRL will convert the test procedure to a metric 
format while making it as compatible as possible with a nearly completed heat pump standard of the International Standards Organization (ISO). Methods for addressing operating features of newer heat pumps, like history-dependent defrost schemes, will also be pursued. Finally, a few parts of the present test procedure, such as default values used in the calculations, will be re-evaluated.

4. Evaluate if an indoor fan delay appreciably and consistently affects SEER and HSPF.

5. Provide DoE recommendations for future work on developing a generic test procedure for heat pumps that incorporate thermal storage and domestic water heating.

Appliance performance ratings are used by consumers when buying, by electric utilities in devising rebate programs, and by governments for promoting or mandating the installation of high efficiency appliances.

\section{Recent Results}

ASHRAE Standard 137P to be released for public review in April 1994.

\section{Development of Revised Test Pro- cedures for Refrigerator-Freezers}

Principal Investigator:

James Kao, (301)975-5871

Building Environment Division

301-975-5871

\section{Sponsor: \\ Department of Energy \\ Office of Building Technologies \\ Codes and Standards Division}

\section{Objective}

To develop revised test procedures on residential refrigerator-freezers and use this data to assess feasibility of future revisions to the Department of Energy (DoE) test procedures for refrigeratorfreezers.

\section{Problem}

The current DOE test procedure was developed nearly 10 years ago. This test procedure includes certain test conditions and assumptions which may not be realistic operating conditions (e.g., 32.2 $\pm 1^{\circ} \mathrm{C}$ ambient temperature is presently required during the test and the door-opening is required only for the variable defrost control optional test) resulting in higher energy consumption rates than field test results. More advanced manufacturing techniques (lower anti-sweat heater energy, better cabinet insulation, better door gaskets, etc.), and operational features (e.g., electronic defrost operation, through-the-door ice service, etc.) are now used extensively. A thorough review of the full test procedure is needed.

\section{Approach}

BFRL conducted tests on two refrigerator-freezers la top-freezer unit and a side-by-side door unit) in FY 1993 to determine the sensitivity of room temperature, room humidity, anti-sweat heater, and door openings on energy consumption. During FY 1994, BFRL will expand work to test room temperature and door openings at multi-levels to determine refrigerator-freezer energy use. BFRL also will conduct tests on automatic ice making and defrost characteristics. BFRL will advise DoE on test procedure revisions, if required. An updated and improved energy test procedure which reflects refrigerator-freezer using conditions more realistical- 
Iy will benefit consumers in their purchasing decisions.

Preliminary analysis of sensitivity tests indicates that room temperature and door openings have significant effect on refrigerator-freezer energy consumption. Room humidity and anti-sweat heater have minor effect than the other two parameters. Future tests should be concentrated on varying room temperature and door openings.

\section{Recent Results}

Completed development and performed check out tests of the test facility.

\section{Test Procedures for Plumbing Products}

\author{
Principal Investigator: \\ Stephen J. Treado \\ Building Environment Division \\ 301-975-6444
}

Sponsor:
Department of Energy
Office of Building Technology
Codes and Standards Division

Objective

To provide testing and rating procedures for deter-

mining performance of plumbing products.

\section{Problem}

The Energy Policy and Conservation Act (PL 94163) (EPCA), as amended, required the Department of Energy (DOE) to prescribe test and rating procedures and minimum performance standards for various residential appliances. In addition, the 1987 amendments to EPCA requires analysis of any test procedure amendments to determine their effect on minimum efficiency standards. DOE has, since 1975 , relied on BFRL to assist in the development of the test and rating procedures.

\footnotetext{
Approach

During FY 1994, BFRL will continued providing DOE with technical assistance in support of proposed Rule Making. This work includes providing technical support on standards and test procedures and attending industry meetings and standards testing plumbing products to demonstrate conformance to the standards. This work will promote the use of more water conserving plumbing products.

Recent Results

Developed proposed rule for testing and certifying covered plumbing products.
} 


\section{Development of Revised Test Pro- cedures for Pool and Spa Heaters}

\author{
Principal Investigator: \\ Stanley T. Liu \\ Building Environment Division \\ 301-975-5880
}

\section{Sponsor:}

Department of Energy

Office of Building Technologies

Codes and Standards Division

\section{Objective}

To develop testing and rating procedure for deter-

mining energy performance of pool and spa heaters.

\section{Problem}

In FY 1993, DOE modified the test procedure for pool and spa heaters to include a calculation of energy factor and published the procedure in the Federal Register as a Proposed Rule Making on August 23, 1993 for public hearing and comments. Comments will be received from industry and other interested organizations during the formal comment period. BFRL will evaluate the comments, make the appropriate revisions, and assist DOE in finalizing the test procedure so it can be published as a Final Rule.

The published DOE test procedure for pool heaters covers only gas- and oil-fired pool heaters. The test procedure references ANSI Standard Z21.56 for gas-fired pool heaters and specifies certain modifications for the procedure to be applicable to oil-fired pool heaters. It does not cover electric resistance type heaters or the heat pump pool heaters which are recently introduced into the market place. A revision to the published test procedure is needed to include the installation, measurements, test methods, and calculation procedures for both the electric resistance type and the heat pump heaters.

\section{Approach}

Over the last 2 years, the ASHRAE SPC 146P committee has developed the ASHRAE Standard $146 \mathrm{P}$ for testing and rating pool and spa heaters that include fossil fueled and electrical operated heaters. The standard includes the installation, instrumentation, test methods and procedures, and calculation procedures for all types of heaters in a single document. In FY 1993, BFRL actively partici- pated in the development of this draft standard and provided comments and suggestions over several versions of the standard. The draft standard was recently been approved by ASHRAE SPC 146P for public review and comment.

During FY 1994, BFRL will continue to provide comments and suggestions during the review process so that the final standard can be referenced in a new, revised DOE test procedure for pool and spa heaters.

The development of this test procedure is expected to provide a uniform method of test on the energy performance of all pool heaters so that similar appliances can be rated equally and fairly and their compliance to the minimum energy standards determined.

\section{Recent Results}

Participated in the development of the draft standard by the ASHRAE SPC 146P for pool and spa heaters. 
BUILDING ENVIRONMENT DIVISION

\section{INDOOR AIR QUALITY}




\section{Development and Application of Multizone Indoor Air Quality Models}

\author{
Principal Investigator: \\ Andrew K. Persily \\ Building Environment Division \\ 301-975-6418

\section{George Walton} \\ Building Environment Division \\ 301-975-6421
}

\section{Sponsor:}

National Institute of Standards and Technology

\section{Objective}

To develop an advanced airflow and contaminant dispersal model in the CONTAM series (BFRL's multizone airflow and contaminate dispersal model) specifically CONTAM94, investigate options for integrating multizone airflow and contaminant analysis into a comprehensive indoor air quality model, apply CONTAM94 to indoor air quality analysis in support of revising ASHRAE Standard 62-1989.

\section{Problem}

Multizone airflow and contaminant models have been developed at BFRL, including AIRNET and CONTAM86 through CONTAM93. These models were unique at the time of their development and were useful for the study of airflow and indoor air quality in multizone building systems. As the indoor air quality field continues to improve, there lacks a comprehensive building air quality model that considers component model development, system development, and integrated system development. Component model development refers to the development of models to describe specific airflow and contaminant dispersal processes for incorporation into a general model as CONTAM93. Examples include aerosol transport and nonlinear chemical reaction. System development refers to efforts to improve the approaches used to form and solve the systems of equations within a general model like CONTAM93. Integrated system development refers to the integration of airflow, contaminant dispersal, thermal and other types of models into a comprehensive indoor air quality model. A comprehensive model is needed to assess indoor air quality prob- lems by risk assessors, building designers, producers of building materials and furnishings, HVAC equipment manufacturers, and indoor air quality researchers. In the near term, the impending revision of ASHRAE Standard 62 will present immediate needs for building air quality modeling capabilities.

\section{Approach}

During FY 1994, BFRL will perform three tasks:

1. Improve CONTAM93 through the development of CONTAM94. CONTAM93 will be evaluated for its existing component models, the user interface and its potential for integration with other types of analysis. Based on this evaluation, the highest priority areas for further development will be identified and pursued in the development of CONTAM94. It is expected that improvements will be made in the ventilation system and the source and sink models.

2. Preliminary implementation of approaches to link airflow and contaminant dispersal analysis with thermal building analysis. This work includes the integration of airflow and contaminant dispersal analysis with building thermal analysis. The various possibilities for integration will be investigated including: the further development of CONTAM94 to include thermal analysis and other types of analysis; the implementation of CONTAM 94 by linking its input and output with other programs within another program such as TRNSYS; and using simulation environments to develop a fully integrated indoor air quality analysis tool. Preliminary attempts to develop integrated tools will be pursued to evaluate the appropriateness of these different approaches.

3. The application of these models to indoor air quality and building energy use issues of immediat $\epsilon$ interest. CONTAM94 and some of the preliminary integrated models will be used to study indoor air quality problems of interest. These problems will be selected based on the activities of the ASHRAE committee responsible for the revision of ASHRAE Standard 62-1989, Ventilation for Acceptable Indoor Air Quality.

\section{Recent Results}

Developed CONTAM93, an improved version with a graphic interface for input and output and uses advanced airflow analysis algorithms. 
Hon, George W., CONTAM193 User's Manual, NISTIR 5385, National Institute of Standards and Technology, Gaithersburg, MD, April 1994.

\section{Development of Protocols for Ventilation System Performance Evaluation}

Principal Investigator:

Andrew K. Persily

Building Environment Division

301-975-6418

W. Stuart Dols

Building Environment Division

301-975-5860

\section{Sponsor:}

National Institute of Standards and Technology

\section{Objective}

To identify needs for standardized protocols for evaluating ventilation system performance for use in building air quality studies and to develop evaluation protocols to meet these needs.

\section{Problem}

Currently, inadequate attention is focussed on ventilation system performance in research projects and diagnostic investigations of building air quality problems. Need all standardized protocols to assess ventilation in buildings at a level of effort appropriate to indoor air quality studies. While ventilation assessment procedures have been developed by the HVAC and the testing, adjusting, and balancing (TAB) industries, these procedures are too complex for use in indoor air quality studies. Research level procedures using tracer gases also are available, but these are still too complex and expensive for wide application.

\footnotetext{
Approach

During FY 1994, BFRL will assess the needs of the HVAC, testing, adjusting, and balancing industry customers for ventilation evaluation protocols. The assessment involves obtaining and analyzing response from these practitioners to BFRL's developed checklists and manual developed in FY 1993. Based on this effort, a list of needs for ventilation evaluation protocols will be developed in the areas of diagnostics, preventive maintenance, and research. In addition, manufacturer's of instrumentation to evaluate ventilation system performance will be contacted to discuss their products, existing protocols, user needs and future products to meet
} 
these needs. A workshop will be held at NIST to bring together these manufacturers, the users of ventilation assessment protocols, representatives of relevant industry associations, and researchers. At this workshop, the participants will discuss the need for different ventilation evaluation protocols and the instrumentation needed to implement these protocols. Based on the results of this workshop, BFRL will identify and define ventilation evaluation protocols that need to be developed. Draft protocols will be prepared and submitted to ASTM or ASHRAE committees as appropriate for development into standards.

Recent Results

Persily, Andrew K., Manual for Ventilation Assessment in Mechanically Ventilated Commercial Buildings, NISTIR 5329, National Institute of Standards and Technology, Gaithersburg, MD, January 1994.

\section{Infiltration/Ventilation - Large Buildings}

\author{
Principal Investigator: \\ Andrew K. Persily \\ Building Environment Division \\ 301-975-6418
}

Sponsor:

Department of Energy

Office of Building Technology

Objective

To develop and demonstrate equipment and procedures for assessing ventilation in public and commercial buildings in conjunction with indoor air quality investigations and understand the relationship between envelope airtightness and commercial building design and construction.

\section{Problem}

Indoor air quality investigations often associate poor ventilation system performance with indoor air quality complaints. However, air change rates and other ventilation system performance parameters have not been well characterized in commercial buildings, either as part of these indoor air quality investigations or in general. Many indoor air quality investigations have not included adequate assessments of ventilation, making connections between ventilation performance and indoor air quality even more difficult to establish. There is a lack of a reliable procedures for assessing ventilation system performance at a practical level of effort and expertise. Most of the research into ventilation system performance has involved sophisticated tracer gas techniques that are not consistent with the resources and technical skills of most indoor air quality investigators. Excessive envelope air leakage impacts indoor air quality, envelope material durability, and occupant comfort. However, current thermal loads analysis programs are not capable of integrating multi-zone airflow analysis. Hence, the energy impacts of envelope air leakage and poor ventilation system control are not known.

Approach

During FY 1994, BFRL will perform three tasks:

1. Develop and field test an automated system for monitoring ventilation system performance parameters. This work involves field deployment of an 
automated ventilation monitoring system, to advance knowledge of the performance of ventilation systems in commercial buildings and to evaluate the applicability of this system to the evaluation of ventilation system performance. This monitoring system, developed by BFRL during FY 1992, is based on the real-time monitoring of key ventilation system performance parameters. The system will be deployed in the field and the measurement results compared to other, more accurate, measurement systems. The field deployment will provide an opportunity to evaluate the monitoring system's design and performance so it can be modified. In addition, the real-time data on ventilation systems operation and performance will further our understanding of how mechanical ventilation systems perform and thereby assist in the development of evaluation protocols that capture the key aspects of ventilation performance.

2. Participate in industry consensus standards activities, primarily the revision of ASHRAE Standard 62 "Ventilation for Acceptable Indoor Air Quality" and the development of the new ASHRAE Standard 129P "Test Method for Ventilation Effectiveness." In addition, BFRL will participate in ASTM as cochair of subcommittee E6.41 on Infiltration Performance and task group chair of D22.05.01 on Related Factors in Indoor Air Quality.

3. Investigate the relationship between commercial building design and construction and the resultant envelope airtightness. The effort involves planning a multi-phase, multi-year project to investigate the energy impacts of air leakage and ventilation system performance in modern office buildings. The first phase will characterize the interactions between building airflow dynamics and thermal loads. The effort also will include reviewing available analytical tools for analyzing coupled building airflow and energy use to determine the most appropriate approach to achieve the project objective. Also, BFRL will calculate the energy impacts of building leakage and poor ventilation system control using the simplified approach of available analytical tools.

The results of this work will support the activities of the EPA BASE Program by developing new approaches to large building monitoring and by improving the usefulness of existing ventilation evaluation procedures.

\section{Recent Results}

Persily, A.K., Building and HVAC Characterization for Commercial Building Indoor Air Quality Investiga- tions, NISTIR 4979, National Institute of Standards and Technology, Gaithersburg, MD, 1992.

Persily, A.K., "Assessing Ventilation Effectiveness in Mechanically Ventilated Office Buildings," Proceedings of International Symposium on Room Air Convection and Ventilation Effectiveness, University of Tokyo, July 1992. 


\section{Radon Entry and Mitigation in Large Buildings}

Principal Investigator:

Andrew K. Persily

Building Environment Division

301-975-6418

\section{Sponsor:}

Environmental Protection Agency

Office of Radiation Programs

\section{Objective}

To evaluate radon entry and air movement in a large, nonindustrial building.

\section{Problem}

There has been much research conducted on the subject of radon in buildings. This research has focused primarily on problem identification, mitigation, and source modeling in single-family residential buildings. Recently, attention has turned towards large buildings such as schools, commercial buildings, and multi-family residential buildings. While the fundamental issues regarding the relationship between radon, ventilation, and building features in small buildings has not been resolved, radon in large buildings is a more complex problem due to the multi-zone nature of airflow and contaminant dispersal in large, mechanically ventilated buildings. The technical issues that must be studied to understand radon transport in large buildings include the impacts of mechanical ventilation system operation, air movement via vertical shafts within tall buildings, and pressure differences at ground contact zones.

\section{Approach}

During FY 1994, BFRL will study radon transport in large buildings through multi-zone airflow and contaminant dispersal modeling. BFRL's CONTAM88, multizone aiflow and contaminant dispersal model, will be applied to four large buildings: a twelve-story multi-family residential building, a mechanically ventilated office building with a decentralized ventilation system, a mechanically ventilated office building with a centralized system, and a single-story school building. A large number of simulations will be run in each building to study the effects of envelope airtightness, the airtightness of interior partitions, ventilation system operation, and the form of the radon source model.
Recent Results

Investigated radon source models for use in the large building modeling effort and developed pressure-dependent and pressure-independent models and incorporated them into the CONTAM 88 input files for the four buildings. 


\section{Study of IAQ Improvement Through the Use of HVAC Systems}

Principal Investigator:

Andrew K. Persily

Building Environment Division

301-975-6418

Steven J. Emmerich

Building Environment Division

301-975-6459

\section{Sponsor:}

Consumer Product Safety Commission

Directorate of Engineering Sciences

Division of Mechanical Engineering

Objective

To assess the impact of existing HVAC technology on residential indoor air quality and assess the potential for using HVAC systems to reduce indoor pollutant levels.

\section{Problem}

Despite the increasing interest in residential indoor air quality problems, little research has been conducted to analyze the impact of pollutant sources, residential HVAC system operation and building envelope leakage on indoor pollutant levels. Most of the research to date has employed simple models of the building and its systems, ignoring the multizone nature of the airflows involved. The use of such simple analytical procedures has limited our understanding of the impact of HVAC systems and other systems on residential indoor air quality and the possibility of using these and other systems to mitigate some indoor air quality problems.

\section{Approach}

During FY 1994, BFRL will focus attention to completing the second phase of a two-phase project. In Phase I of this project, BFRL completed three tasks: conducted a literature review, developed a plan for computer analysis, and held a peer review meeting to discuss this plan. During FY 1994, BFRL will implement the computer simulations developed last year.

During FY 1994, BFRL will perform three tasks of this second year of a two year effort:
1. Calculate concentrations of selected indoor contaminants given the baseline houses and pollutant sources. This data will provide baseline pollutants for comparing the effectiveness of the IAQ control retrofits.

2. Design the IAQ control retrofits and their incorporation into the model houses.

3. Perform preliminary computer simulations to determine the effectiveness of the retrofits. At the discretion of CPSC, this effort will be followed by a Phase $\mathrm{B}$ effort where the computer simulations are completed and analyzed.

\section{Recent Results}

Emmerich, Steven J. and Persily, A.ndrew K., Indoor Air Quality Impacts of Residential HVAC Systems Phase / Report: Computer Simulation Plan, NISTIR 5367, National Institute of Standards and Technology, Gaithersburg, MD, February 1994. 


\section{Indoor Air Quality Commissioning Program for TWFN}

\author{
Principal Investigator: \\ Andrew K. Persily \\ Building Environment Division \\ 301-975-6418
}

\section{Sponsor:}

Nuclear Regulatory Commission

Office of Consolidation

\section{Objective}

To develop and apply an indoor air quality commissioning program to the new Nuclear Regulatory Commission office building to ensure an acceptable workplace to the building occupants.

\section{Problem}

The Nuclear Regulatory Commission is in the process of constructing a new office building in the greater Washington, DC area. Based on experiences in other buildings, NRC is concerned about the possibility of experiencing indoor air quality problems in the new building associated with movein. These concerns center on the "staged" occupancy approach that will be employed in the building, in which a portion of the building will be occupied while other areas in the building are still be finished. In addition, the activity of moving in the occupants and their belongings can be associated with unusual contaminant sources. The occupancy of new office buildings has the potential for indoor environmental problems associated with new building materials. While many strategies have been proposed to reduce the potential for such problems, the technical bases for these approaches are limited. There has been insufficient sufficient research on air quality in new, or existing, buildings to develop properly documented procedures for preparing new office buildings for occupancy.

\section{Approach}

During FY 1994, BFRL will develop and implement indoor air quality commissioning procedures. This work will consist of several stages including an evaluation of the ventilation system design, initial indoor air quality monitoring during construction, the development of criteria for the acceptability of the space prior to and immediately after occupancy, and acceptance of spaces within the building for occupancy based on these criteria. During the initial monitoring effort, BFRL will evaluate the ventilation system design and performance and measure selected indoor pollutants during the interior finishing. The design will be compared with current practice and relevant standards for its adequacy to address indoor air quality concerns. The pollutant level measurements will be used to anticipate the existence of unusual pollutant sources associated with building inaterials or interior furnishings. While there are currently few standards for such sources, the measurements will be compared with the results of measurements in other buildings.

Following this work, BFRL will develop criteria for the acceptability of a portion of the building for occupancy. These criteria will be based on the results of on-site inspections, tests of the ventilation system performance and indoor pollutant concentration measurements. Criteria will include outdoor air delivery rates, pressure relationships between spaces, and indoor concentrations of particulates, formaldehyde, radon and volatile organic compounds. The post-occupancy criteria will include indoor carbon dioxide levels.

When the BFRL criteria has been accepted by NRC, they will be applied in the building to spaces as they become ready for occupancy. BFRL will inspect the space and the HVAC system and conduct indoor contaminant measurements. The results of the inspection and measurement efforts will be compared to the agreed upon criteria to determine the acceptability of the space. Roughly one month after the space is occupied, BFRL will return to the building to reinspect the space and HVAC system and to conduct follow-up measurements. The results will then be compared to the post-occupancy environmental criteria to determine if the space is still acceptable. This procedure for determining acceptability will be applied to the building until it is fully occupied.

\section{Recent Results}

New project. 


\section{Indoor PAH and Particulate Emissions from Wood Stoves}

\author{
Principal Investigator: \\ Andrew K. Persily \\ Building Environment Division \\ 301-975-6418

\section{Sponsor:} \\ Consumer Product Safety Commission \\ Directorate of Health Sciences
}

\section{Objective}

To measure indoor levels of selected polycyclic aromatic hydrocarbons (PAHs) and particulates associated with wood stoves in order to determine the emission rates of these substances under typical residential use.

\section{Problem}

Benzo[a]pyrene (BaP) and other PAHs associated with wood stove emissions have previously been investigated for the cancer risk associated with these substances. Based on these risks, the U.S. Environmental Protection Agency (EPA) set requirements to reduce particulate emissions into outdoor air from wood stoves. While CPSC has performed a cancer risk assessment from exposure to $\mathrm{PAH}$ in the indoor air from the previous generation of nonairtight stoves, they need to determine indoor PAH levels associated with the new generation of wood stoves complying the EPA's Phase II (final) emission requirements.

\section{Approach}

During FY 1994, BFRL will measure the levels of $\mathrm{PAH}$ and particulate emissions from wood stoves complying with EPA's Phase II requirements for particulate emissions. These tests will be performed in a test house, and the stoves will be operated consistent with typical residential use. During the tests, BFRL will measure: 1 . BaP, including particulate and vapor phases combined; 2 . up to 10 other individual PAHs, including particulate and vapor phases combined; 3. total particulate-phase PAHs with a "real time" PAH monitor provided by CPSC; 4. particulate matter below 10 micrometers in diameter (PM10); 5. continuous particulate counts; and 6 . building air change rates measured with automated tracer gas decay. In addition to these quantities, indoor and outdoor environmental conditions will be monitored during the tests.
BFRL staff will instrument the test house, conduct the tests, collect the PAH samples and perform the particulate, air change rate and environmental measurements. The analysis of the PAH samples will be performed by NIST's Chemical Science and Engineering Laboratory. The measured data will be analyzed to enable the reporting of average concentrations, air change rates, and environmental conditions over periods of several hours during the tests.

\section{Recent Results}

New project. 
BUILDING ENVIRONMENT DIVISION

\section{COMPUTER INTEGRATED CONSTRUCTION}




\section{Advanced Manufacturing Systems and Networking Testbed (AMSANT) for the Process Plant Industries}

Principal Investigator:

Kent A. Reed

Building Environment Division

301-975-5852

\section{Sponsor:}

National Institute of Standards and Technology

\section{Objective}

To create an experimental computing and communication facility for establishing a baseline of industrial practice in process plant engineering and construction, for serving as a open testbed for integration activities of industrial partners, and for serving as a communications hub for U.S. participants.

\section{Problem}

The process plant industries comprise highly competitive vendors and users of computerized systems for the design, construction, and maintenance of process plants. An open facility is needed in which these players can test proposals for integrating their computerized systems without bias or unnecessary disclosure of proprietary developments. This research supports a companion project, STEP for the Process Plant Industries, for testing the information models proposed for Standard for the Exchange of Product Model Data (STEP) application protocols.

\section{Approach}

This is a multi-year project. During FY 1994, BFRL will perform four tasks:

1. Create the laboratory space, procure equipment, and install a communications network.

2. Develop a plan for linking the distributed components into a NIST-wide AMSANT facility in collaboration with other NIST laboratories.

3. Establish electronic mail list and file servers to link participants in the STEP Process Plant Application Protocol projects.

4. Install at least two major software packages that address process plant design and construction activities; develop a data dictionary for the two systems; and build representations of a publicdomain model of a process plant piping system in the software packages.

The experimental computing and communication facility will be a distributed component of the NISTwide Advanced Manufacturing Systems and Networking Testbed (AMSANT).

This work will provide process plant owners, contractors, and software vendors with the open testbed needed for testing and verifying technical approaches being taken in the development of STEP. The emerging U.S. industry consortium known as PLANT/STEP is expected to be an early user of this testbed.

Recent Results

New project. 


\section{Process Plant Engineering and Construction: Structural Systems}

Principal Investigator:

Long Phan

Structures Division

301-975-6077

\section{Sponsor:}

National institute of Standards and Technology

\section{Objective}

To develop the technical basis for a virtual construction environment, based on the Standard for the Exchange of Product model data (STEP) technologies, that supports the design and construction of structural systems for process plants.

\section{Problem}

The U.S. process plant industries seek to improve their use of computerized systems through integration, e.g., automation of the exchange and sharing of information among systems. Effective integration will enable the introduction of virtual construction environments by allowing engineers and constructors to visualize and manipulate a common process plant model from widely disparate viewpoints. In this environment, the plant design can be optimized for constructability and maintainability. The evolving international standard ISO 10303---Product Data Representation and Exchange, known as STEP, is providing the base technology needed for integration. STEP application protocols must be developed that meet the needs of the process plant industries, notably in the creation of virtual environments.

\section{Approach}

This is a multi-year project.

During FY 1994, BFRL will:

1. Develop a test case of structural steelwork, piping and equipment, sufficiently complex to be representative of process plants and sufficiently detailed to support structural analysis and detailed structural design.

2. Select and review existing application reference models for structural systems that have been brought to the STEP project, including the ship structural systems model from the Navy/Industry Digital Data Exchange Standards Committee (NIDDESC), and the structural steelwork for build- ings model from the European Strategic Program for Research and Development in Information Technologies (ESPRIT) CIMSTEEL project.

3. Critique the models using the test case and propose refinements and extensions needed to support the creation of a virtual construction environment.

4. Work with the companion project---AMSANT for the Process Plarıt Industries---to plan a prototype implementation of the test case.

This research will establish a technical baseline and verify test case data for the STEP application protocols that are being developed nationally. The emerging U.S. industry consortium known as PLANT/STEP is expected to be an early partner in this research.

Recent Results

New project. 


\section{STEP for the Building Industry}

Principal Investigator:

William F. Danner

Building Environment Division

301-975-5855

Mark E. Palmer

Building Environment Division

301-975-5858

Sponsor:

National Institute of Standards and Technology

\section{Objective}

To demonstrate automated information exchange using STEP (Standard for the Exchange of Product model data) in the building industry.

\section{Problem}

National and International standards bodies have adopted the application protocol (AP) methodology for developing information exchange standards. The USA IGES/PDES Organization and the ISO TC $184 /$ SC4, which is developing the Standard for the Exchange of Product Model Data (STEP), are proponents of this new method.

BFRL initially developed the AP methodology as a solution for ensuring reliable information exchange using the Initial Graphics Exchange Specification (IGES). With Industry and other government agencies, BFRL developed the 3D Piping IGES Application Protocol, both to prove the methodology and to meet a high-priority data exchange requirement of industry and government.

There is now world-wide activity to develop industry-specific application protocols in the ISO STEP project. Recently, ISO TC 184/SC4 approved the creation of the Process Plant Application Protocol Planning Project. A framework is needed for determining what application protocols should be developed for chemical process plants. U.S. Industry input is needed to ensure that the application protocols will be useful when completed. An experimental software environment is needed for testing the ideas and information requirements that are advanced in the project.

\begin{abstract}
Approach
During FY 1994, BFRL will establish a U.S. industry advisory panel. Working with this panel and with the Process Plant Application Protocol Planning project in the ISO STEP project, BFRL will develop prototype information requirements for a functional/schematic description of a chemical process plant, comparable to a process and instrumentation diagram (P\&ID), and for a physical description, comparable $t$ the traditional plastic model. BFRL will apply the planning framework developed last year to determine the suite of application protocols to be developed in the project. BFRL will develop an experimental database implementation of an application reference model (ARM) for a prototype application protocol relating to chemical process plants.
\end{abstract}

The results of this research are expected to provide owners and contractors with the information needed to select appropriate approaches for describing process plants consistently and completely using STEP application protocols.

\section{Recent Results}

Palmer, Mark E. and Gilbert, Mitch, Guidelines for the Development and Approval of STEP Application Protocols, NISTIR 5110, National Institute of Standards and Technology, Gaithersburg, MD, November 1993. 


\section{STEP for the Process Plant Industries}

\author{
Principal Investigator: \\ Mark E. Palmer \\ Building Environment Division \\ 301-975-5858
}

\section{Sponsor:}

National Institute of Standards and Technology

\section{Objective}

To assist the U.S. process plant industries develop the Standard for the Exchange of Product model data (STEP) application protocols needed for sharing and exchanging information during the design and construction, and maintenance of plant piping and equipment systems.

\section{Problem}

The U.S. process plant industries seek to improve their use of computerized systems through integration, e.g., automation of the exchange and sharing of information among systems. The many computerized systems in use can be integrated only at great cost because of their incompatible proprietary representations of information; information exchange today is accomplished largely through manual methods. Standard, neutral information representations and exchange methods are needed that allow system vendors to be innovative and yet allow system users to exchange and share information about process plants automatically. The evolving international standard ISO 10303---Product Data Representation and Exchange, known as STEP, is providing the base technology. STEP application protocols must be developed that meet the needs of the process plant industries.

\section{Approach}

In a BFRL workshop conducted in FY 1992, owners, engineers and constructors, information technology providers, and parts suppliers identified plant piping systems as the key focus of application protocol development. They also recommended establishment of a preliminary technical work plan to address the development of a product data model to support 1. the selection and use of commodity items, 2. the development of piping and instrumentation (P\&l) diagrams from process stream information, 3. the development of piping system geometry from $P \& l$ information, 4 . the capture of project management data, 5. demonstrating compliance with EPA, OSHA, and ISO 9000-series standards, and 6 . the exchange of piping system data from designer to fabricator.

BFRL will address these issues in priority order as established in further workshops, through interactions with industry consortia and individual companies, and through the STEP Process Plant Application Protocol Planning Project (PPAPPP). This is a multi-year project. BFRL will assess the state of information technology practice in the process plant industries; refine the existing process plant activity model that has been submitted to the STEP PPAPPP by the U.S. Process Data Exchange Institute (PDXI); and work with industry to generalize the application reference model for ship piping systems developed by the Navy/Industry Digital Data Exchange Standards Committee (NIDDESC). BFRL will work with researchers at the University of Missouri-Rolla to assess $\mathrm{P} \& \mathrm{I}$ information requirements. BFRL, in collaboration with the industry consortium PlantSTEP, will develop the Group I documentation for a STEP application protocol for process plant piping systems information. BFRL will continue to co-lead the STEP Process Plant Application Planning Project in ISO TC184/SC4/WG3.

Recent Results

Palmer, Mark E. and Gilbert, Mitch, Guidelines for the Development and Approval of STEP Application Protocols, NISTIR 5110, National Institute of Standards and Technology, Gaithersburg, MD, November 1993.

NIST workshop on automated information exchange in Construction: Process/Petrochemical Industries and Piping Systems, held in October 1992.

Palmer, Mark E. and Reed, Kent A., 3D Piping IGES Application Protocol Version 1.1, NISTIR 4797, National Institute of Standards and Technology, Gaithersburg, MD, March 1992. 


\section{Computerized Maintenance Management Information}

\author{
Principal Investigator: \\ Kent A. Reed \\ Building Environment Division \\ 301-975-5852
}

Sponsor:

National Institute of Standards and Technology

Objective

To develop digital data standards that facilitate the exchange of information with computerized maintenance management systems.

\section{Problem}

A major impediment to the implementation of computerized maintenance management systems is the high cost of manually gathering, formatting, and inputting the required information about the building and its systems. Typically, this information is available only in the paper forms such as "as-built" drawings, specifications, and manufacturer's data sheets and manuals. Furthermore, current computerized maintenance management systems typically have different and often proprietary data formats that prevent automatic exchange of information from one to another, as from a work order scheduling system to a spare parts inventory system. Frequently, these exchanges are performed manually and at another high cost.

In FY 1993, an ASTM Task Force, E06.25.11, was created to consider standardization of computerized maintenance management information. This task force took as its first priority the development of a standard that facilitates the entry of manufacturer's operation and maintenance information about building equipment such as chillers, boilers, and elevators. Recognizing that complete capture of all possible information is still an unsolved technical problem, the task force agreed first to standardize on the structure of such information and to develop a document tagging scheme that enforces this structure. The Standard Generalized Markup Language, SGML (ISO 8879), is under consideration as the implementation mechanism.

\section{Approach \\ During FY 1994, BFRL will develop and demonstrate trial SGML Document Type Definitions (DTD's) that}

capture the document structures developed by the ASTM task force. Successful completion of this work will validate the technical approach taken by ASTM E06.25.11, enabling manufacturers to deliver equipment, maintenance information electronically from the same databases used to generate traditional printed documentation and enabling building operators to read electronic information directly into maintenance management systems.

Recent Results

New project. 


\section{Prototype Requirements Management System}

\author{
Principal Investigator: \\ Mark E. Palmer \\ Building Environment Division \\ 301-975-5858
}

\section{Sponsor:}

Department of Defense

Office of the Defense CALS Executive

\section{Objective}

To develop a prototype Requirements Management System for use by DoD and industry to define and prioritize its requirements for STEP (Standard for the Exchange of Product Model Data).

\section{Problem}

DoD, other government agencies, and industry need a comprehensive process for documenting requirements and for ensuring that these requirements are incorporated into the standards development process.

\section{Approach}

During FY 1994, BFRL will develop a functional specification and technical evaluation criteria for a prototype RMS based on its investigation of software tools for requirements management and the successful demonstration of a trial requirements management system (RMS) performed last year. The prototype RMS will be developed and tested in conjunction with the NIST National PDES Testbed APs for DoD and Industry Project. The results of this test will be used to improve the RMS. Based on the success of this test, this project will assess the utility of applying the RMS to the $\mathrm{CIC}$ work on information systems for the Process Plant Industries.

The results of this research are expected to provide industry and government agencies with costeffective methods and tools for collecting, synthesizing, and establishing consensus on requirements for information systems.

\section{Recent Results}

Developed trial Requirements Management System (RMS) and electronic questionnaires, tested trial RMS in conjunction with the CALS project to develop a Technical Data Package Application
Protocols Suite, and delivered electronic questionnaires to DoD program managers and technical experts.

Palmer, Mark, Judd, Jon, Hodges, John, and Crusey, Jesse, Requirements for a Digital Technical Data Package Application Protocol Suite (TDP AS), NISTIR, National Institute of Standards and Technology, Gaithersburg, MD (in preparation).

Palmer, Mark, Hodges, John, Judd, Jon, and Crusey, Jesse, Project Plan for a Digital Technical Data Package Application Protocol Suite for DoD and Industry, NISTIR, National Institute of Standards and Technology, Gaithersburg, MD (in preparation). 
BUILDING ENVIRONMENT DIVISION

\section{HEAT TRANSFER}




\section{Test Procedures for Advanced Thermal Insulation Products}

\author{
Principal Investigator:
}

A. Hunter Fanney

Building Environment Division

301-975-5864

\section{Sponsor:}

National Institute of Standards and Technology

\section{Objective}

To evaluate thermal measurement techniques applicable to advanced insulation systems

\section{Problem}

Advanced insulation products are being developed and introduced into the U.S. market. Examples of these products are aerogels, powder filled panels, and evacuated panels. These products offer extremely high insulating capabilities. For example, theoretical calculations reveal that an $\mathrm{R}$-value approaching 100 may be achieved in a $25.4 \mathrm{~mm}$ thick evacuated panel.

Unfortunately, current test procedures for measuring thermal conductivity are only applicable to materials which are homogenous, have planar surfaces, and have, compared to advanced insulation products, relatively poor insulating capabilities. Appropriate measurement techniques are needed which will allow an accurate determination of the overall thermal conductance of advanced insulation systems.

\section{Approach}

During FY 1994, BFRL will conduct parametric analyses using ANSYS (software package used to conduct finite element difference computations) to assess the effect of convective film coefficients and test panel aspect ratios. The convective coefficients on the inside and outside of the panel under evaluation will be determined using test panels of known thermal conductivities and temperature measurements of surface and bulk air temperatures. Software will be developed to permit the acquisition of pixel by pixel temperature data of IR images which can subsequently be used to compute the total convective heat loss from an advanced insulation panel.
BFRL's calorimetric facility will be completed. This includes adding power supply and measurement equipment capable of measuring DC power levels on the order of 15 watts. Instrumentation will be added to measure the bulk air, panel surface, and mask surface temperatures at numerous locations. User-friendly software will be developed to control the experiments and acquire the experimental data.

Homogenous test panels will be constructed. The thermal conductivity of these panels will be measured using BFRL's Guarded Hot Plate Facility. The panels will subsequently be used in the calorimetric facility to assess measurement error and to determine the convective coefficients present on both sides of the test panel. Actual advanced insulation panels will be measured using both the calorimetric technique and using the IR system. The use of thermochromatic crystals and a color imaging system also will be explored.

The expected benefit of this research will be a method of test which will permit an accurate determination of the performance of various types of advanced insulation systems.

\section{Recent Results}

Entered into a Cooperative Research and Development Agreement (CRADA) with Aladdin Industries, a compact vacuum insulation manufacturer. The CRADA will provide a means by which BFRL can secure compact vacuum insulation systems of various sizes and construction. BFRL will provide Aladdin Industries with insight on how to design insulation systems with greater thermal resistance based on the finite element modeling results. 


\section{Test Methods for Evaluating the Thermal Conductivity of Small Insulation Samples}

\author{
Principal Investigator: \\ Robert R. Zarr \\ Building Environment Division \\ 301-975-6436
}

Sponsor:

National Institute of Standards and Technology

Advanced Technology Program

\section{Objective}

To assess and develop a measurement method for determining the thermal conductivity of small-size insulation specimens.

\section{Problem}

Economic factors dictate that the initial development of the next generation of thermal insulation materials require small-size specimens for evaluation. For example, specimen sizes may be on the order of a $6 \mathrm{~mm}$ diameter disk. Current steadystate test methods require relatively large, planar specimens for determining thermal conductivity and may not be suitable for small specimens. Other more recent methods, such as the transient needleprobe or the laster-flash method may be more suitable methods for determining the thermal conductivity of small-size specimens.

\section{Approach}

During FY 1994, BFRL will conduct a survey of the literature, industry, and academia to identify methods to determine the thermal conductivity of small samples. The suitability of each method will be evaluated by an assessment of the accuracy and practical utility of each measurement method. Measurement methods, to be considered, include the transient needle-probe, the line or strip heatsource, the laser-flash technique, and infrared analysis. Based on the assessment, a small-scale apparatus will be designed and developed using the most promising technique. In addition, measurements of small specimens will be conducted during this year. The results of this work will be used by manufacturers and designers to evaluate the thermal conductivity of the next-generation of thermal insulations.
Recent Results

New project. 


\section{Thermal Insulation SRM for Wall or Window Test Methods}

\section{Principal Investigator:}

Robert R. Zarr

Building Environment Division

301-975-6436

\section{Sponsor:}

National Institute of Standards and Technology

Office of Standards Reference Materials

\section{Objective}

To develop a thermal insulation Standard Reference Material (SRM) for use in a wall or window test apparatus.

\section{Problem}

Recent interlaboratory studies conducted under the auspices of the National Voluntary Laboratory Accreditation Program (NVLAP) and ASTM have demonstrated a need for a thermal insulation SRM suitable for use in calorimetric hot-box wall tests. Such an SRM is also desired by the National Fenestration Rating Council (NFRC) for test methods for windows.

\section{Approach}

During FY 1994, BFRL will consult with members from industry to identify sources for the candidate SRM. A small quantity of candidate material (one to five specimens) will be selected. A set of preliminary thermal conductivity measurements at $297 \mathrm{~K}$ will be conducted to evaluate the candidate material using BFRL's $1 \mathrm{~m}$ guarded-hot-plate. Based upon a survey of industry's test apparatus, BFRL will acquire about 250 to 500 specimens of the candidate SRM. The experimental design for characterizing the material is a full factorial of 15 thermal conductivity measurements; 5 temperatures and 3 densities. BFRL will consult with NIST's Computing and Applied Mathematics Laboratory for the experimental design.

This project will provide a new standard reference material that will assist two industries. These include the window manufacturers as well as the thermal testing laboratories of many insulation manufacturers.

\section{Recent Results}

New project.

\section{Thermal Resistance Measurements on Foam Insulation and Powder- Panel Insulation}

\author{
Principal Investigator: \\ Robert R. Zarr \\ Building Environment Division \\ 301-975-6436
}

\section{Sponsor:}

Department of Energy

Oak Ridge National Laboratory

Office of Building Technology

Building Systems Division

\section{Objective}

To determine the thermal resistance of cellular foam insulation and powder-filled evacuated panel (PEP) insulation using the guarded hot plate (ASTM C 177) and heat-flow-meter apparatus (ASTM C 518).

\section{Problem}

Manufacturers of refrigerator appliances are examining alternative insulations to cellular plastics for insulating the walls of their refrigerators. One alternative under consideration is evacuated panels of micro-porous powder that can obtain a resistivity of 70 to $140(\mathrm{~m} \cdot \mathrm{K}$ per $\mathrm{W})$. While providing a high resistance, these panels pose a difficult measurement problem because of increased potential of lateral heat flow from the meter area. Assessing the accuracy of the measurement for these evacuated panels is important in designing the future generation of refrigerator appliances.

Foam insulations are cellular plastics that entrap a gaseous blowing agent having a low thermal conductivity. Over time, ambient gases will permeate the foam while conversely, the blowing agent can escape. The net effect is a reduction in the performance of the foam insulation. ORNL and others have developed a thin slicing accelerated aging test for foam insulation. NIST has been requested by ORNL to participate in an interlaboratory comparison on determining the longterm thermal performance of a rigid DIR foam.

\section{Approach}

During FY 1994, BFRL will perform measurements on foams and powder-filled evacuated (PEP) insulation. These measurements will be made after 
consultations with ORNL staff and may require ORNL to supply additional specimens to BFRL. An interlaboratory comparison will provide data for verifying the accuracy of the thin slicing technique. The data can also be included in the precision and bias statements for ASTM standards.

\section{Recent Results}

Performed four measurements of thermal resistance at ambient conditions as part of an inter-laboratory "round-robin" with ORNL, Certain-Teed Corporation, and Owens-Corning Fiberglas, Inc.

\section{Experimental Validation of NIST Moisture Transfer Model}

\author{
Principal Investigator: \\ Robert R. Zarr \\ Building Environment Division \\ 301-975-6436
}

\section{Sponsor:}

National Institute of Standards and Technology

\section{Objective}

To experimentally verify BFRL's Moisture Transfer Model for a wide range of different wall constructions and quantify the effect of accumulated moisture on the heat transmission under both steady and dynamic conditions.

\section{Problem}

BFRL has developed a distributed-moisture-capacity, one-dimensional, transient finite-difference model, called MOIST, that predicts the coupled transfer of heat and moisture within multilayer construction under nonisothermal conditions. The model predicts moisture transfer in both the diffusion and capillary flow regimes. It has a provision to account for convective moisture transfer by including embedded cavities which may be coupled to indoor and outdoor air. This model uses hourly WYEC weather data, and predicts the average moisture content of the layers of a wall as a function of time of year. There is a need to experimentally verify this model.

\section{Approach}

During FY 1994, BFRL will install representative $1 \mathrm{X}$ $1 \mathrm{~m}$ wall specimens and instrument it in its calibrated hot box. The exterior surfaces of the wall specimens will be exposed subsequently to four conditioning periods: 1 -month steady $7^{\circ} \mathrm{C}$ winter condition, 1-week dynamic winter condition, and 2-week steady $32.2^{\circ} \mathrm{C}$ summer drying condition. The dynamic condition will consist of a $16.7^{\circ} \mathrm{C}$ peak-topeak sine wave having an average value of $7^{\circ} \mathrm{C}$. During the three conditioning periods, the interior surfaces of the wall specimens will be exposed to ambient air at $24^{\circ} \mathrm{C}$ and $50 \%$ rh.

During the three conditioning periods, the moisture accumulation within the exterior layers of the wall specimens and the rate of heat transfer at the interior surfaces will be measured as a function of 
time and compared to corresponding values predicted by the MOIST model.

In designing the experiment, wall specimens will be selected that verify BFRL's MOIST model (heat and moisture transfer computer model) as a function of the following parameters: the thermal resistance and moisture storage capacity of the insulation, the permeance of the vapor retarder, the permeance of interior and exterior paint layers, and the permeance of the sheathing. In addition, walls representative of current construction practice will be included in the study. Each of these walls will be instrumented with moisture content sensors for measuring the moisture content of the exterior layers, thermocouples for measuring the temperature distribution, and a heat-flux transducer for measuring the rate of heat transfer at the interior surface.

This research will assist the building community develop recommended practices for controlling moisture in buildings. The model may be used with confidence by building designers and engineers to conduct moisture sensitivity analysis to develop recommended practices for controlling moisture accumulation in building construction.

\section{Recent Results}

Burch, D.M., Thomas, W.C., and Fanney, A.H., "Water Vapor Permeability Measurement of Common Building Materials," ASHRAE Transactions, Vol. 98, Part 2, 1992.

Richards, R.F., Burch, D.M., and Thomas, W.C., "Water Vapor Sorption Measurements of Common Building Materials," ASHRAE Transactions, Vol. 98, Part 2, 1992.

Burch, D.M. and Thomas, W.C., "An Analysis of Moisture Accumulation in a Wood Frame Wall Subjected to Winter Climate," Proceedings of the Thermal Performance of the Exterior Envelopes of Buildings V, Clearwater, Florida, December 1992.

\section{Controlling Moisture in Walls/Roofs of Manufactured Housing}

\author{
Principal Investigator: \\ Douglas M. Burch \\ Building Environment Division \\ 301-975-6433
}

Sponsor:

Department of Housing and Urban Development Policy Development and Research

Affordable Housing Research \& Technology Division

Objective

To enhance BFRL's heat and moisture transfer model (MOIST) and use the enhanced model to investigate the effectiveness of practices for controlling moisture in the walls and roof cavities of manufactured housing.

\section{Problem}

During FY 1993, BFRL used its sophisticated computer program, called MOIST, to develop recommended practices for controlling moisture in walls and roofs of manufactured housing. HUD staff felt that certain limiting assumptions of MOIST may have affected BFRL's previous analysis. HUD requested that these limiting assumptions be removed from MOIST and the enhanced model be used to investigate the viability of the previously developed recommended practices.

\section{Approach}

During FY 1994, BFRL will incorporate the following models into Program MOIST: 1. a detailed air flow model that predicts the exfiltration air flow through the ceiling and roof construction of a manufacture home, 2. a moisture balance model that predicts indoor relative humidity as a function of outdoor climate; and 3. a model that predicts the ventilation rate of a roof cavity as a function of wind speed.

With the above enhancements, MOIST will predict the storage of moisture in the construction materials of roof and wall constructions as a function of time of year. The computer model will show how different variables effect the moisture level in a roof cavity. For example, air flow from the indoor space into the roof cavity will be modeled for various ceiling air tightnesses ranging from a tight to a leaky ceiling. The computer model will include the following two effects of attic ventilation 1. remov- 
ing moisture from moist materials of an attic and 2. its potential to increase air leakage from the indoor space into a roof cavity. This model will be able to resolve the contentious issue as to whether attic ventilation provides a "net" reduction in moisture accumulation of construction materials in a roof cavity. In addition, the modei will include the effect of indoor ventilation on moisture accumulation in the building envelope.

Using the enhanced model, BFRL will perform a series of cold-climate and hot-and-humid climate computer runs to investigate the effectiveness of recommended practices for controlling moisture in the walls and roof cavities of a current-practice manufactured home (i.e., without supplemental ventilation to comply with the ASHRAE Ventilation Standard 62). This analysis should resolve the issue as to whether natural ventilation of a roof cavity is an effective moisture-control practice and whether other proposed practices for controlling moisture are effective.

A separate series of computer runs will be carried out to investigate whether new-practice homes (i.e., with supplemental indoor ventilation to comply with ASHRAE Standard 62) can be operated without attic ventilation. Here a constant indoor ventilation rate of $0.35 \mathrm{ACH}$ would be maintained. An interior vapor retarder would be installed in the ceiling construction with the air leakage sites sealed.

BFRL also will use MOIST to investigate the possibility of developing an innovative wall and roof cavity construction that performs satisfactorily in both cold climates and hot and humid climates.

This research will lead to improvements and revisions to the HUD Manufactured Home Construction and Safety Standards for better controlling moisture in the building envelopes of manufactured housing.

\section{Recent Results}

Developed proposed practices for controlling moisture in the walls and roof cavities of manufactured housing for cold climates and hot and humid climates.

Burch, D.M. and TenWolde, A., Controlling Moisture in the Walls of Manufactured Housing, NISTIR 4981, National Institute of Standards and Technology, Gaithersburg, MD, December 1992.
Burch, D.M., Controlling Moisture in the Roof Cavities of Manufactured Housing, NISTIR 4916. National Institute of Standards and Technology, Gaithersburg, MD, November 1992. 


\section{Moisture Control Guidelines for Attics, Cathedral Ceilings, Crawl Spaces, and Walls}

\author{
Principal Investigator: \\ Douglas M. Burch \\ Building Environment Division \\ 301-975-6433
}

\section{Sponsor:}

Department of Energy

Office of Building Technologies

Building Systems Division

\section{Objective}

The objectives of this project are to conduct a comprehensive one-dimensional analysis of the combined heat and mass transfer in attics and cathedral ceilings, develop guidelines and practices for preventing moisture problems in attics and cathedral ceilings, enhance MOIST to include a twodimensional algorithm for predicting the heat and mass transfer in the earth beneath a crawlspace, and develop a user's manual for MOIST to permit building engineers and architects to evaluate strategies for controlling moisture in hotel/motel building envelopes.

\section{Problem}

Appropriate moisture control guidelines for attics, cathedral ceilings, and crawlspaces are still debated by experts in the building community. For example, DOE recently issued a Moisture Control Handbook. The Technical Oversight Committee for this Handbook sometimes could not reach a consensus on certain guidelines and practices. A factor contributing to this situation is that climate has a profound influence on effectiveness of a particular practice. For example, a ceiling vapor retarder and attic ventilation are recommended in cold climates to minimize moisture accumulation in roof sheathing. However, these same practices are counter productive in attics of air conditioned houses exposed to hot and humid climates. In this situation, attic ventilation increases the amount of moisture introduced from the outdoor environment into an attic, and a ceiling vapor retarder prevents moisture from passing through the construction and thereby causes moisture to accumulate at that location. Similar considerations apply to cathedral ceilings and crawlspaces.
Field studies are useful because they document a specific moisture problem as it relates to a particular building construction. Moreover, they often provide an understanding of the underlying physics. However, it is difficult to generalize individual field experiments to different constructions, outdoor climates, and indoor conditions. This is because complex inter-actions exist between the construction materials and the indoor and outdoor climates. It is impractical to carry out an adequate number of field experiments to cover all possible constructions and indoor and outdoor climates.

\section{Approach}

BFRL's personal computer program, MOIST, predicts the combined transfer of heat and moisture in multi-layered building construction under nonsteady-state conditions. MOIST predicts the moisture content and temperature of construction layers as a function of time of year. It includes moisture transfer by vapor diffusion and capillarity. The program inputs a user-defined construction and hourly WYEC ASHRAE weather data which is available for 51 different cities of the United States and Canada.

During FY 1994, BFRL will perform four tasks:

1. Attics and Cathedral Ceilings. BFRL will use MOIST to investigate the viability for all attic and cathedral ceiling constructions given in the DOE Moisture Control Handbook. The moisture content of the construction materials will be predicted and plotted as a function of time of year. Hourly weather data for the following climates will be used in the analysis: a cold winter climate (Madison, WI), a mixed climate (Washington, DC), and a hot and humid climate (Lake Charles, LA). Based on the results of this analysis, guidelines and practices will be developed to control moisture problems in attics and cathedral ceilings. These computer simulations will resolve the contentious issue as to whether attic ventilation provides a "net" reduction in moisture accumulation of attic construction materials. Attic ventilation, unquestionably, removes and transports moisture from moist attic materials to the outdoor environment. However, attic ventilation also may increase air leakage from the indoor space into an attic, thereby increasing the moisture load into the attic.

2. Crawlspaces. BFRL will enhance MOIST to include 2-dimensional algorithms to predict the 
combined transfer of heat and moisture in the earth beneath a crawlspace. The modes of moisture transfer will include diffusion and capillarity. This enhanced model will be tested using one crawlspace detail of the Moisture Control Handbook for a mixed climate and one for a cooling climate. A study will be designed to develop guidelines and practices for controlling moisture problems in crawl spaces as a function of climate. This study will be conducted during the following year. A literature review will be conducted to find moisture properties for soils.

3. Measurement of Moisture Properties of Ten Roofing Materials. Newly developed BFRL measurement methods will be used to measure spot permeability curves (permeability versus relative humidity) and sorption isotherms (moisture content versus relative humidity) for seven roofing materials. These measurements will support ongoing research at the Oak Ridge National Laboratory (ORNL).

4. Envelope Moisture Control Strategies. Develop a Recommended Procedure for Evaluating Envelope Moisture Control Strategies during Hotel/Motel Guest Room Design and Construction. MOIST can be used to simulate typical constructions used in hotels/motels. BFRL will prepare a user's manual describing how to use MOIST. This manual will be targeted at an architectural/engineering firm involved in the design and construction of building envelopes.

This research will lead to improved guidelines and practices for controlling moisture in various parts of building envelopes. This, in turn, will reduce moisture-related material degradation, thereby increasing the long-term durability of building envelopes.

\section{Recent Results}

Verified MOIST by comparing it to a series of laboratory experiments using a calibrated hot box.

Burch, D.M., "An Analysis of Moisture Accumulation in Walls Subjected to Hot and Humid Climates," ASHRAE Transactions, v99, part 2, 1993.

Burch, D.M. and TenWolde, A., "A Computer Analysis of Moisture Accumulation in the Walls of Manufactured Housing," ASHRAE Transactions, v99, part 2, 1993. 
BUILDING ENVIRONMENT DIVISION

\section{LIGHTING TECHNOLOGY}




\section{Field Measurements and Modeling of Lighting Distributions}

\author{
Principal Investigator: \\ Belinda L. Collins \\ Building Environment Division \\ 301-975-6455
}

Stephen J. Treado

Building Environment Division

301-975-6444

\section{Sponsor:}

National Institute of Standards and Technology

\section{Objective}

To develop a procedure for evaluating lighting quality based on luminance distribution, including the measurement system, the technical basis for the lighting quality metric, and the requirements for computer modeling.

\section{Problem}

Evaluating and predicting the performance of lighting systems in actual office spaces is a complicated measurement and modeling problem requiring both physical and user reaction assessments. Accurate measurement and prediction procedures for determining lighting system performance in real spaces are urgently needed, along with metrics for evaluating and predicting lighting quality as related to luminance distributions. Recent research has suggested that the perception of lighting quality is determined in part by the balance between horizontal and vertical illumination.

\section{Approach}

During FY 1994, BFRL will develop measurement procedures and metric to assess lighting quality. This work involves three tasks:

1. Developing the measurement system and procedure for collecting and processing the luminance distribution information in typical building spaces. This work includes determining measurement requirements such as spatial resolution and greyscale resolution, and methods for assembling hemispherical luminance distributions from limited field-of-view images.

2. Developing the technical basis for an index or metric of lighting quality, based on luminance distribution. This work involves correlating subjec- tive assessments of lighting conditions to associated physical measurements of luminous conditions for a range of lighting designs and configurations.

3. Determining the requirements for computer simulation of luminance distributions to enable prediction of lighting quality. This work involves determining the level of modeling detail needed to provide the luminance distribution resolution necessary for determining the lighting quality metric.

It is expected that the results of this work will expand the analysis of lighting system performance and lighting quality metrics to real rooms with different lighting systems, surface reflectances, and interior obstructions using a luminance mapping device. Data obtained with the mapper will be used to develop and verify the accuracy of computer algorithms for computing both surface luminances and reflectances in real rooms, including those with obstructions.

Recent Results

Conducted concurrent evaluations of luminance distributions, occupant subjective responses and visual performance for a range of lighting system configurations. 


\section{Lighting and HVAC Interactions}

\author{
Principal Investigator: \\ Stephen J. Treado \\ Building Environment Division \\ 301-975-6444
}

\author{
Sponsor: \\ Department of Energy \\ Office of Building Technologies \\ Building Systems Division
}

\section{Objective}

To develop improved evaluation methods and design tools for the design and operation of efficient lighting and HVAC systems in commercial buildings to control peak cooling loads.

\section{Problem}

Lighting constitutes a substantial portion of commercial building electrical energy usage, typically ranging from 25 to $50 \%$. Most of the energy dissipated to the building space eventually contributes to building cooling load. Due to the temperature dependence of fluorescent lighting power consumption and light output, there are significant interactions between the lighting and HVAC systems. As a result, lighting system light levels and efficiency, and cooling loads due to lighting, can vary substantially due to lighting and HVAC system design and operation. Previous results have indicated performance variations of up to $20 \%$ are possible. These effects influence the number of luminaires required to provide the design illumination levels and the size of HVAC equipment to meet the cooling loads contributed by the lighting system. This, in turn, influences the first cost of the lighting and HVAC systems, and the operating costs for energy, including demand charges for electrical power usage during peak periods. Such peak period power usage puts added pressure on electrical utilities to meet system-wide power demand by increasing generating capacity.

\section{Approach}

During FY 1994, BFRL will investigate the interactions between lighting and HVAC systems in commercial buildings through a combination of fullscale measurements and computer simulations. The results are being analyzed to provide the technical basis for design procedures and methods. Full-scale measurements are being conducted at the BFRL
Lighting Interaction Test Facility for various lighting system, HVAC system and room configurations, and operating conditions. Lighting system performance, thermal loads, and energy transfers are monitored to enable determination of optimum operating conditions and strategies. A detailed computer model is being developed and verified using the measurement results. The model will be used to extend che results to a wide range of configurations so that the design guidelines will be more general and comprehensive. The computer model will serve as a stand-alone design tool, but also will be the basis of a sub-routine of larger building energy analysis computer programs such as BLAST and DoE-2. This will provide such programs with the capability of evaluating the interactions between the lighting and HVAC systems, a capability which they currently lack. This work will provide design data and procedures to enable the selection and implementation of energy efficient lighting systems, and to provide methods for controlling peak cooling loads.

\section{Recent Results}

Morris, F., Braun, J., and Treado, S., "Experimental and Simulated Performance of Optical Control of Building Thermal Storage," ASHRAE Technical Paper, New Orleans, January 1994. 


\section{Evaluation of Occupant Response to Lighting Retrofits}

\author{
Principal Investigator: \\ Belinda L. Collins \\ Building Environment Division \\ 301-975-6455

\section{Sponsor: \\ Department of Energy \\ Office of Building Technologies \\ Codes and Standards Division}

\section{Objective}

To evaluate occupant response to the lighting in offices at DOE Headquarters in Washington, DC.

\section{Problem}

The Department of Energy is renovating the lighting system in its Headquarters as part of the Federal Relighting Initiative. This initiative is intended to reduce power consumption dramatically without compromising existing lighting quality. Determining the impact of the relighting on the occupants and on the lighting in their offices is a major part of the evaluation of the effectiveness of the relighting initiative. Recent reports by Collins, Fisher, Gillette, and Marans (1990), Collins and Rubin (1988), Collins, Gillette, Dahir and Goodin (1989) present data from post-occupancy evaluations of about 15 facilities in both government and private industry. These assessments provided information on physical conditions, especially lighting, and the occupant reaction to it in the various buildings studied. Data from post-occupancy evaluations of several U.S. Army facilities suggested that lighting levels were below IES minimum recommendations for the types of tasks done in the facilities. Occupant satisfaction was also below that obtained in other areas in the same facility. These data were used to suggest areas where lighting retrofits could be effective and the types of situations that could be improved. Similar information is needed for the retrofits planned for the Forrestal Building to determine their overall effectiveness.

\section{Approach}

During FY 1994, BFRL will determine representative samples of offices to assess any daylight contribution and conventional and open-plan offices. A two-part procedure is planned in which physical measures of the lighting in the offices are assessed, including measures of task illuminance, surround luminances, and typical task contrasts; and occupant reaction is determined using questionnaire techniques developed in previous BFRL post-occupancy assessments. The number of measures required to characterize and describe the space will be determined using a conventional photometer and a luminance mapping device in conjunction with a statistical analysis procedure. After the photometric measures have been obtained, the spaces will be evaluated by human subjects using post-occupancy procedures similar to those described in Collins et al (1989). This will provide baseline information on the conditions existing before the renovation and data on the effectiveness of the renovation in improving lighting conditions in the offices. It will allow the occupant response to be associated with relevant physical conditions to provide information on which changes were particularly effective and suggest areas where further renovation might be needed. During the course of the project, a comprehensive procedure for measuring luminances in an office space will be implemented, along with a protocol for assessing occupant response. These procedures will be documented and validated during the assessment of the Forrestal Building for future use in other Federal Relighting Projects. Development of repeatable, valid procedures for measuring the effectiveness of relamped facilities will enable DOE to provide critical feedback information to building managers. This work will provide objective and subjective assessments of the effectiveness of a significant retrofit of a commercial office lighting system, providing lighting designers with much needed useful information on lighting retrofit performance.

\section{Recent Results}

Identified 250 typical spaces, administered questionnaires, and physical measurements were made in the spaces both prior to relamping and postretrofit. Results are being evaluated to identify correlations between occupant response and lighting conditions. 


\section{Test Procedures for Lighting Fixtures and Systems}

Principal Investigator:

Steve Treado

Building Environment Division

301-975-6444

\section{Sponsor:}

Department of Energy

Office of Building Technologies

Codes and Standards Division

\section{Objective}

To provide equitable testing and rating procedures for determining energy performance of lighting fixtures and systems.

\section{Problem}

The Energy Policy and Conservation Act (P 94-163) (EPCA), as amended, required the Department of Energy (DOE) to prescribe test and rating procedures and minimum performance standards for various residential appliances. In addition, the 1987 amendments to EPCA requires analysis of any test procedure amendments to determine their effect on minimum efficiency standards. DOE has, since 1975 , relied on BFRL to assist in the development of the test and rating procedures.

\section{Approach}

During FY 1994, BFRL will perform three tasks:

1. Support DOE in Rule Making on the Interim Final Rule specifying test procedures for incandescent reflector lamps. BFRL will evaluate the comments, make the appropriate revisions, and assist DOE in finalizing the test procedure so it can be published as a Final Rule.

2. Assist DOE clarify which lamps are exempt from standards in the Energy Policy Act of 1992 by specifying definitions of color temperature, rough service, etc. BFRL also will recommend a sampling plan for testing lamps to demonstrate conformance to the standards. No sampling plan is specified in the 1992 law.

3. Continue work on developing luminaire performance testing and labeling, and testing procedures for HID lamps. BFRL will assess the adequacy of the luminaire test procedures suggested by the voluntary luminaire labeling program, and work with NEMA, IES, and NVLAP on the selection of luminaire categories for testing. Luminaire performance will be investigated by making detailed laboratory measurements. For HID lamps, existing test procedures will be evaluated for their adequacy and comprehensiveness, with any gaps or inconsistencies identified. Laboratory measurement capability will be established for HID lamps. This work will provide the technical basis for evaluating and characterizing the performance of lamps and luminaires as a means of promoting energy efficiency.

\section{Recent Results}

Treado, S., Testing Procedures for Multilamp Luminaires, NISTIR 5262, National Institute of Standards and Technology, Gaithersburg, MD, April 1994. 
FIRE SAFETY ENGINEERING DIVISION

\section{FIRE PROTECTION APPLICATIONS}




\section{Large Scale Smoke Movement}

Principal Investigator:

John H. Klote

Fire Safety Engineering Division

301-975-6890

Sponsor:

National Institute of Standards and Technology

\section{Objective}

To develop and verify algorithms for modeling large scale smoke movement in buildings.

\section{Problem}

The simulation of smoke movement in HAZARD (BFRL's model that predicts the hazard to a building and occupant anywhere within a building) has limited applicability to large spaces such as corridors, atria, and shafts. The algorithms developed by this project will allow realistic simulation of smoke transport in these spaces. The applicability of the HAZARD methodology will be extended to large scale smoke flow including office buildings, apartment buildings, homes for the elderly, and Navy and merchant ships.

\section{Approach}

During FY 1994, BFRL will be addressing the following four topics:

Shaft. FLOW3D la commercial package computer model that simulates fluid dynamics and heat transfer using a finite element difference approach) will be used to gain an understanding of the mechanisms, i.e., wall attachment, smoke mixing, plug flow, etc., involved with smoke transport through open shafts with concurrent and counter flows expected to occur in buildings due to the natural forces of building flow. Based on this mechanism study, a shaft smoke flow strategy will be developed including user needs of a shaft model, flow phenomena, and model concept. Work will start on a first order model for open shafts, and flow in stairwells will be addressed later in this project.

Atrium. This topic involves the development of a method to predict smoke movement in a stratified environment and an atrium/top-shaft venting algorithm in modular form for adaption into CFAST (BFRL's building fire model), and compare simula- tions with existing data from venting experiments. Later, the model will be verified against FLOW3D.

Corridor. BFRL will conduct FLOW3D simulations including heat transfer to study smoke flow in corridors. The results of these simulations will be compared to the hybrid model to validate the model.

Wall Heat Transfer. The wall heat transfer model, developed in FY 1993, for FLOW3D will be compared against the standard heat transfer routines of FLOW $3 D$ and RAD3D (three dimensional radiation) to study the efficiency and accuracy of BFRL's new model. The final report of this heat transfer model will include this comparison.

The information gained from the study smoke movement in shafts, atrium, and corridors will form a foundation to develop smoke movement modeling techniques to evaluate hazards in large buildings.

Recent Results

Cooper, L.Y., VENTCF2: An Algorithm and Associated Computer Software for Calculating Flow Through a Horizontal Ceiling/Floor Vent in a ZoneType Compartment Fire Model, NISTIR, National Institute of Standards and Technology, Gaithersburg, MD; (under development and to be submitted for publication in the Journal of Fire Protection Engineering, 1994).

Cooper, L.Y., Simulating Smoke Movement Through Long Vertical Shafts in Zone-Type Fire Models, NISTIR, National Institute of Standards and Technology, Gaithersburg, MD; (under development and to be submitted for publication in Fire Safety Journal, 1994).

Cooper, L.Y., Combined Buoyancy- and PressureDrive Flow Through a Horizontal Circular Vent, NISTIR, National Institute of Standards and Technology, Gaithersburg, MD, lunder development to be submitted for publication in the Journal of Heat Transferl.

Cooper, L.Y., "Calculating Combined Buoyancy- and Pressure-Driven Flow Through a Horizontal, Circular Vent; Application to a Problem of Steady Burning in a Ceiling-Vented Enclosure," to be submitted to Fire Safety Journal, 1994. 
Klote, J.H. and Forney, G.P., Zone Fire Modeling with Natural Building Flows and a Zero Order Shaft Model, NISTIR 5251, National Institute of Standards and Technology, Gaithersburg, MD, 1993.

Klote, J.H., A Method for Calculation of Elevator Evacuation Time, Journal of Fire Protection Engineering, 5, 3, pp 83-96, 1993.

Klote, J.H., Design of Smoke Control Systems for Areas of Refuge, ASHRAE Transactions, American Society of Heating, Refrigerating and Air Conditioning Engineers, 99, 2, pp 793-807, 1993.

Yamada, T. and Cooper, L.Y., Experimental Study of the Exchange Flow Through a Horizontal Ceiling Vent in Atrium Fires, Abstracts; Annual Conference on Fire Research, October 13-15, 1992, NISTIR 4924, National Institute of Standards and Technology, Gaithersburg, MD, 1993.

Cooper, L.Y., Combined Buoyancy-and PressureDriven Flow Through a Horizontal Vent: Theoretical Considerations, NISTIR 5252, National Institute of Standards and Technology, Gaithersburg, MD, September 1993.

Klote, J.H., Deal, S.P., Levin, B.M., Groner, N.E., and Donoghue, E.A., Workshop on Elevator Use During Fires, NISTIR 4993, National Institute of Standards and Technology, Gaithersburg, MD, 1992.

Matsushita, T. and Klote, J.H., Smoke Movement in a Corridor - Hybrid Model, Simple Model and Comparison with Experiments, NISTIR 4982, National Institute of Standards and Technology, Gaithersburg, MD, 1992.

\section{Grants}

"Movement of Smoke and Hot Gases in Open Shafts," Jaluria, Yogesh, Rutgers, State University of New Jersey.

\section{Study of Heat and Smoke Movement and Their Influence on Detector and Sprinkler Response in Enclosed Spaces with Complex Ceiling Geometries}

\author{
Principal Investigator: \\ Richard W. Bukowski \\ Fire Safety Engineering Division \\ 301-975-6853
}

\section{Sponsor:}

National Fire Protection Research Foundation

General Services Administration

Public Building Services

Office of Real Property Management and Safety

Federal Emergency Management Agency

U.S. Fire Administration

\section{Objective}

To examine the effect of complex ceiling geometry and obstructions on the distribution of heat and smoke to optimize requirements for automatic fire detector location found in NFPA 72E and for automatic sprinklers as covered by NFPA 13, 13D, and $13 R$.

\section{Problem}

At present, recommendations for the placement of automatic fire detectors cited in the NFPA Standard on Detection Devices (72E) are based on engineering judgement or on experiments performed by Heskestad and Delichatsios on flat, unobstructed ceilings. In the implementation of these data, tables and curves were developed from calculations with a zone model which are also only applicable for flat, unobstructed ceilings. Since $72 \mathrm{E}$ includes recommendations for placement of fire detectors in spaces with sloping or peaked ceilings, or with open beams and joists, some validation of these recommendations is necessary. Further, detector citing problems associated with stratification and high air movement from HVAC systems are mentioned in the standards, but only limited installation guidance is provided which are based only on judgment. 
Approach

During FY 1993 and 1994, the first 2 years of the study, work centers on examining the effects of fire size, fire location, ceiling height, ceiling slope, typical ceiling beam or joist configurations, and the effect of stratification on the distribution of heat and smoke from the fire. In the second 2 years, FY 1995 and FY 1996, research will address the effects of HVAC systems on fire detectors and sprinkler activation.

Specific geometries to be examined have been selected to coincide with experimental studies identified in the literature review conducted during FY 1993; providing validation for the calculations. Additional geometries will be added in consultation with the Technical Advisory Committee of the project sponsors. ASHRAE will contribute their technical assistance in those tasks relating to HVAC effects and to identify specific HVAC system configurations to maximize the applicability of results.

The work is designed to be applicable over a wide range of detector types and installation characteristics. Detector types will include those that respond to heat, smoke, and gas. The work will address numerical modeling using a field model, the Harwell Flow3D (commercial computer model that simulates fluid dynamics and heat transfer using a finite difference approach) to simulate the room geometries and fire growth; selected experimental verification will be included when needed.

The model will be used to produce contour plots of temperature, velocity, and smoke concentration near the ceiling at the level of the sensing components of typical detectors and sprinklers. These detailed distributions will then be used to develop engineering guidelines for detector/sprinkler placement, resulting in recommendations for changes to the appropriate NFPA standards. Validation of the numerical results for the project will be performed using a combination of existing fire experiments and, as necessary, by conducting additional experiments. The work is directly translated into changes to codes for the installation of fire detectors and sprinklers. Improved fire codes mean better performing and more cost effective protection system.

\section{Recent Results}

Forney, G.P., Bukowski, R.W., and Davis, W.D., Field Modeling Effects of Flat Beamed Ceilings on
Detector and Sprinkler Response, National Fire Protection Research Foundation, Quincy, MA, 1993. 


\section{Balanced Design}

\author{
Principal Investigator: \\ Richard W. Bukowski \\ Fire Safety Engineering Division \\ 301-975-6853
}

\section{Sponsor:}

National Institute of Standards and Technology

General Services Administration

Public Building Services

Office of Real Property Management and Safety

Federal Emergency Management Agency

U.S. Fire Administration

\section{Objective}

To quantify the performance and reliability of fire detection systems, sprinklers, and compartmentation as directed by the U.S. Fire Administration Act of 1992, PL102-522.

\section{Problem}

Trade-offs between active and passive fire protection strategies are often made without technical foundation. As Federal legislation mandates use of sprinklers and detectors in specified occupancies, the term, compartmentation, must be understood so overall safety is not compromised. PL102-522 required BFRL to conduct this study as a joint effort of the pubiic and private sectors.

Approach

During FY 1994, BFRL will perform this work as four tasks:

1. Collect data on the field performance and reliability of fire protection systems for office and hotel occupancies, BFRL will work with insurance interests, e.g., Factory Mutual, IRI, Cigna, and American Insurance Services Group to collect similar data on other occupancies as background information to enrich the database. BFRL will work with industry representatives to identify other major systems users who might represent sources of field data. Besides the basic difference in fire resistance ratings, analyzed field data will identify other characteristics of these construction types which impact on their mitigation of risk.

2. Develop analytical framework to evaluate the risk mitigation contribution of life safety, property protection, and business continuity systems. This effort will use the parameter lists from related Fire Safety Evaluation Systems (FSES), incorporating quantitative calculation methods wherever possible, and converting the output measures to risk mitigation objectives. As the framework evolves, data needs which are identified will be incorporated in work performed in task 1.

3. In task 1, types of system impairments which reduce the ability of the system to perform will be identified. To the extent that these reduce but not prevent performance, special studies may be needed to quantify this reduction. Modeling and testing of impaired assemblies will be performed. 4. One of the study's goals is to determine the conditions under which one or more of the systems can be reduced or eliminated without unacceptable risk of loss. This is likely to be a highly subjective measure which needs a public policy type of decision. A panel consisting of building owners, insurance officials, and regulators will be convened to help achieve a consensus view of types and levels of losses considered acceptable to society.

This work is expected to produce a sound basis for making trade-off decisions in codes and for determining performance equivalencies for alternative designs.

\section{Recent Results}

Bukowski, R., Balanced Design Concepts Workshop, NISTIR 5264, National Institute of Standards and Technology, Gaithersburg, MD, 1993. 


\section{Risk Assessment/Performance Codes}

Principal Investigator:

Richard W. Bukowski

Fire Safety Engineering Division

301-975-6853

\section{Sponsor:}

National Institute of Standards and Technology

\section{Objective}

To develop a risk-based method for establishing the degree to which fire safety features and systems contribute toward the goals of a performance-based code.

\section{Problem}

Parallel efforts are underway in several countries to develop risk-based methods to assess compliance with performance codes. These methods incorporate identical frameworks and cite the same set of calculational procedures for the major portions of the calculations. Differences occur primarily in data or assumptions and stem from local experience. There is great interest among the participants to collaborate and harmonize the approaches into a single, standardized method.

\section{Approach}

During FY 1994, BFRL will chair a committee under the auspices of the International Council for Building Research, Studies, and Documentation's (ClB) Working Commission W14 on Fires. A working committee of 20 persons representing 14 countries will contribute to this project. A series of task groups will be organized to develop approaches to specific issues. BFRL will lead the task group working on the development of design safety factors which relate to total computational uncertainty. Differences among the proposed methods will be discussed and consensus reached on compatible approaches.

\section{Recent Results}

Bukowski, R., A Review of International Fire Risk Prediction Methods, Interflam '93, Fire Safety 6th International Fire Conference, Oxford England, Interscience Communications, Ltd., London, England, C.A. Franks, ed., pp 437-466, 1993.

\section{Enhanced GSA Engineering Fire Assezsment System}

\author{
Principal Investigator: \\ Richard W. Bukowski \\ Fire Safety Engineering Division \\ 301-975-6853
}

Sponsor:

General Services Administration

Public Building Services

Office of Real Property Management and Safety

\section{Objective}

To develop and deliver a computer software system, operating under MS-Windows, on Fire Assessment System (FSES) for offices.

\section{Problem}

Current fire safety practice within GSA is rooted in the Life Safety Code prescriptions and the FSES for equivalency determinations. GSA's goal is to move this predictive practice to a risk management basis. GSA is planning a long range program to develop and implement a fire risk management system for their properties by the turn of the century. It will be based on the evolution of the current FSES for offices to a risk basis, underpinned by an advanced FPEtool package and a life-cycle cost evaluation tool.

\section{Approach}

During FY 1994, BFRL will automate the existing FSES for office occupancies in a windows environment and develop plans for the migration of the FSES from a code equivalency to a risk management basis. This program is expected to provide an enhanced tool for GSA to manage fire risk on their properties.

Recent Results New project. 


\section{FPEtool Advancement}

Principal Investigator:

Richard W. Bukowski

Fire Safety Engineering Division

301-975-6853

\section{Sponsor:}

National Institute of Standards and Technology

General Services Administration

Public Building Services

Office of Real Property Management and Safety

\section{Objective}

To develop advance state-of-the-art of analytical tools for the practicing fire protection engineer and to enhance cross compatibility with other analytical techniques and the applicability to hazard and risk analyses.

\section{Problem}

FPEtool (BFRL software used to evaluate hazards and fire protection strategies in GSA Office Buildings) is a highly popular software product among practicing Fire Protection Engineers (FPE's); it is used worldwide. However, FPEtool can not be enhanced further while written in QUICKBASIC. It must be re-written in a structured language $(C)$ and moved into a computer windowing environment.

\footnotetext{
Approach

During FY 1994, BFRL will rewrite the current code in $C$ ior $\mathrm{C}++1$ and moved to Windows or WINDEX. CASE tools will be employed to provide the needed documentation on development for the future. The product development must be coordinated with the development of the GSA Enhanced Fire Safety Evaluation System, which will be risk based. FPEtool is the most widely used fire modeling package in the world. The improved code and manual will allow the continuation of this position.

Recent Results

Developed and released FPEtool v3.2 which included several new routines such as flow down a long corridor, tenabilities areas of refuge, and reducing burning data under sprinkler spray.
}

\section{Modification of Fire Protection Requirements (Performance Based Fire Protection for Nuclear Power Plants)}

Principal Investigator:

Robert S. Levine

Fire Safety Engineering Division

301-975-6671

\section{Sponsor:}

Nuclear Regulatory Commission

\section{Objective}

To provide technical assistance to NRC develop performance based fire safety regulations for nuclear power plants which allow more cost effective operations without sacrificing safety.

\section{Problem}

There is a need to replace prescriptive requirements with alternate means of achieving fire safety that has little impact on safety and that follows a performance based approach.

\section{Approach}

During FY 1994, BFRL will assist NRC prepare a Notice of Proposed Rulemaking to alert the industry to develop and submit proposed performance based fire safety requirements. BFRL will assist NRC in evaluating and refining the industry's proposals. The draft rule is targeted for publication by September 1995.

Recent Results

New Project. 


\section{Emergency Egress from Air Traffic Control Towers (ATCT)}

\author{
Principal Investigator: \\ Richard W. Bukowski \\ Fire Safety Engineering Division \\ 301-975-6853
}

\author{
Sponsor: \\ Department of Transportation \\ Federal Aviation Administration \\ Aviation Safety Division
}

\section{Objective}

To address current deficiencies in code compliance of Air Traffic Control Towers (ATCT) for emergency egress and to develop an approach to longer term issues through the use of protected elevators.

\section{Problem}

The Occupational Safety and Health Administration (OSHA) is enforcing the provisions of the Life Safety Code for ATCT which requires two methods of egress where the occupant load exceeds 25 persons. Currently, FAA's larger towers are in violation. They proposed a code change to permit an increase in load with compensating fire safety features. The FAA requested BFRL technical assistance in its adoption. Simultaneously, the FAA has been requested by the Department of Justice (DOJ) to permit handicapped access to towers under ADA (spell out the Act). This will require a protected elevator which also will serve as a second means of egress for able-bodied persons.

\footnotetext{
Approach

During FY 1994, BFRL will recommend a change to the LSC to recognize a higher occupant load. In a parallel effort BFRL will document the design, performance, and code specifications for protected elevators which could be recognized by the LSC as a second means of egress. The code change will allow the upgrade of existing ATCT's to enhance fire safety for FAA employees. The new code provisions for protected elevators will be more generally applicable and will improve the evacuation capability of both disabled and nondisabled building occupants.
}

Recent Results

New project.

\section{Fire and Thermal Characteristics of Navy Firefighting Trainers}

\author{
Principal Investigator: \\ Robert S. Levine \\ Fire Safety Engineering Division \\ 301-975-6671 \\ Sponsor: \\ U.S. Navy \\ Naval Air Warfare Center \\ Training Systems Division \\ Objective
}

To support the development and implementation of prototype firefighting trainers at Norfolk, VA; Orlando, FL; Mayport, FL; and Bangor, Washington.

\section{Problem}

There is need to develop technical solutions to predict fire damage, toxicity levels, environmental effects, and other developmental problems that affect the safety and operability of prototype trainers. These problems are usually unforeseen, but occur as Navy personnel use the equipment to represent fire problems that were not anticipated in the original design.

\section{Approach}

During FY 1994, BFRL will make the necessary measurements in the prototype trainers such as combustion species concentration, radiant fluxes, unburned fuel gas, duct velocities, etc. BFRL will install experimental monitoring equipment and calculate pollutant dispersion patterns and other factors as required to solve this technical problem.

\section{Recent Results}

Results from this six year level of effort project are routinely presented to the Naval Training Systems Center and to the Naval Project Team meeting on the various trainers. 


\section{Fire Research Information Service}

Principal Investigator:

Nora H. Jason

Fire Safety Engineering Division

301-975-6862

Sponsor:

National Institute of Standards and Technology

Objective

To maintain BFRL's Fire Research Information System (FRIS) as the world's preeminent fire research library and to expand its role to provide access to resources needed to apply fire science findings -- serve as a model for information resource management for the next century.

\section{Problem}

Growth in INTERNET accessibility offers new opportunities to expand FRIS into worldwide data dissemination driven by the use of calculational methods. Offering the user community additional types of databases will be a challenge in hardware, software, and user ability. InFIRE (International network of Fire Information and Reference Exchange) can be central to such expansion as the members represent many fire institutions worldwide.

\section{Approach}

During FY 1994, BFRL will incorporate the new INTERNET capabilities to expand FRIS data dissemination. This expansion will permit the demonstration of electronic access to BFRL documents through electronic linking using software as ACROBAT and its PDF (Portable Document Format). BFRL will establish a prototype FDMS (Fire Data Management System) installation on INTERNET when the software becomes available. During this year, BFRL will near completion of the first phase of FIREDOC (BFRL's bibliographic fire database) collection. FIREDOC contains all BFRL current publications. A separate project is underway to incorporate older publications by the building staff. During the second phase of FIREDOC, BFRL will evaluate the potential for digital storage of collected documents which would provide for reduced storage needs and eventually to remote access pending resolution of copyright issues. Online access would allow the user to identify reports, journal articles, conference proceedings, etc., of interest and be able to view the actual document without leaving the computer. Storage technology which allows full text searching also will be continued.

Recent Results

Upgraded BFRL's FIREDOC computer system which was implemented on INTERNET. This upgrading enhances access from U.S. Universities, government agencies, the private sector, and foreign users. 


\section{Fire Research Bulletin Board System}

\author{
Principal Investigator: \\ Scot Deal \\ Fire Safety Engineering Division \\ 301-975-6891
}

\section{Sponsor:}

National Institute of Standards and Technology

\section{Objective}

To manage and operate BFRL's Bibliographical Fire Research Bulletin Board System (BFRBBS) for disseminating BFRL information, data, and software

\section{Problem}

BFRBBS has been successful as an outreach mechanism, particularly to the fire service. Many users download BFRL's software using the BFRBBS and others use BFRBBS to remain current in their knowledge of BFRL's activities such as its seminar series and annual conference. In order to continuously meet user needs, new information features and capabilities need to be incorporated into BFRBBS.

\section{Approach}

During FY 1993, BFRL upgraded its hardware and software to improve response. During FY 1994 , BFRL will establish special user forums as a means to attract new users and obtain new insights into the use of this software. For example, one forum will address persons performing fire reconstructions; the software will permit them to post questions on availability of data or whether someone tried to model a particular fire condition. Replies also may be anonymous. Other forums will be established for fire service groups (fire department applications) and for code officials who need guidance in evaluating requests for equivalency or variance.

\section{Recent Results}

Upgraded BFRL's hardware and software to facilitate new features and provide more efficient service to users.

\section{Technology Transfer}

\author{
Principal Investigator: \\ Richard W. Bukowski \\ Fire Safety Engineering Division \\ 301-975-6853
}

\section{Sponsor:}

National Institute of Standards and Technology

\section{Objective}

To participate in exhibits at appropriate conferences and provide assistance to agencies requesting to demonstrate BFRL technology applications.

\section{Problem}

BFRL continuously needs to seek methods that reach out to customers and prospective customers to broaden their awareness of BFRL capabilities and breath of BFRL's services.

\section{Approach \\ During FY 1994, BFRL will continue to exhibit its laboratory services to appropriate conferences and shows. During this time BFRL will provide assis- tance to Federal Agencies as opportunities. This year, BFRL will revamp its exhibit with new pro- grammatic displays for each of the five divisions which can be selected for display as a function of audience interest.}

\section{Recent Results}

Participated in the National Fire Protection Administration and Fire Department Instructor's Conference (FDIC), A/E/C Systems Trade Show and Earthquake Engineering Research Institute Conference. 


\section{Fire Research Seminars}

Principal Investigator:

Scot Deal

Fire Safety Engineering Division

301-975-6891

\section{Sponsor:}

National Institute of Standards and Technology

\section{Objective}

To manage the Fire Research Seminar series for presenting a balanced and informative program of presentations on fire technology needs and research that address some of these fire technology needs.

\section{Problem}

BFRL staff need to keep informed of a broad range of technical topics which impact BFRL customer needs and that impact their work. Beyond its own ability to know the current literature, this seminar series needs to disseminate such current information and, at the same time, encourage interactions with researchers and practitioners.

\section{Approach}

During FY 1994, BFRL will continue scheduling its successful Seminar Series and solicit recommendations from experts on appropriate speakers. Work includes scheduling biweekly presentations on timely and relevant topics.

\section{Recent Results}

Hosted successful biweekly Symposia during FY 1993. 
FIRE SAFETY ENGINEERING DIVISION

FIRE MODELING 


\section{Ceiling Fires}

Principal Investigator:

Henri E. Mitler

Fire Science and Engineering Division

301-975-6886

\section{Sponsor:}

National Institute of Standards and Technology

\section{Objective}

To develop an understanding of the effects of the presence of a ceiling on the evolution of a wall fire and develop a validated, documented computer program for CFAST (BFRL's building fire model) and other fire models.

\section{Problem}

The effects of the ceiling on room fires is one of the most notable lacunae in our ability to predict the development of room fires. BFRL's wall-fire project, and other studies, have yielded reasonably successful models which predict wall fires. Most of these models, however, implicitly assume that there is no ceiling, so that the flame can be arbitrarily high. This is true, in particular, for BFRL's computer model SPREAD (which predicts the upward fire spread on walls). In fact, the presence of a ceiling has several important effects. Studying and modeling these effects is necessary to correctly simulate burning in a room.

\section{Approach}

The work is being done as three tasks.

Task 1 was conducted during FY 1993. Here a literature survey was performed of fires impinging on ceilings, including axisymmetric fires, wall fires, and corner fires. Flame extensions, fluxes and their position dependence, entrainment rates, effect on combustion rates, and ignition of flammable ceilings were reviewed. As a result of the survey, it was determined what areas need to be studied further, and it was established how much time and other resources, are needed. A detailed work plan was developed. The theoretical understanding of flows and combustion under ceilings was tied together with empirical observations, and a preliminary algorithm put together. This includes a preliminary plan for writing a stand-alone ceiling fire computer program, to be tied together with the wall-fire computer program SPREAD.
During FY 1994, the second task of this work, BFRL will obtain experimental data to improve the algorithm and incorporate it into BFRL's building fire model, CFAST. BFRL will plan and carry out new experiments. Based on the new data, an improved algorithm will be devised, and a report will document this work.

In task 3, BFRL will calculate the effects of ceiling ignition when the ceiling is flammable, program the algorithm, merge it with SPREAD, and harmonize it with CFAST. Finally, the new program will be validated, publish a report on its research findings, and incorporate the program into CFAST.

The location and construction of the ceiling is one of the determinants of how rapidly an enclosure will flashover, and sometimes whether it flashes over at all. Moreover, understanding this fire behavior will impact on properly predicting the rate of fire spread to adjoining rooms. This work is expected to result in useful stand-alone programs primarily for use in HAZARD (BFRL's model that predicts the hazard to a building and occupant anywhere within a building). Embedding a wall-fire algorithm into a roomfire model such as CFAST (in HAZARD), will give realistic answers when the effects of the ceiling are taken into account.

\section{Recent Results}

Mitler, H.E., An Algorithm to Describe the Spread of a Wall Fire Under a Ceiling, NISTIR in review, National Institute of Standards and Technology, Gaithersburg, MD, 1994 (manuscript in review).

Mitler, H.E. and Steckler, K.D., Comparison of WallFire Behavior With and Without a Ceiling, NISTIR 5380, National Institute of Standards and Technology, Gaithersburg, MD, 1994.

\section{Grants}

"Prediction of Fire Dynamics," Alpert, R. and deRis,

J., Factory Mutual Research Corporation.

\footnotetext{
"Upward Flame Spread on a Vertical Wall," Kulkarni, A., Pennsylvania State University.

"A Study of Fire-Induced Flow Along the Vertical Corner Wall," K. Saito, University of Kentucky.
} 


\section{Fire Model Verification}

Principal Investigator:

Richard D. Peacock

Fire Safety Engineering Division

301-975-6664

\section{Sponsor:}

National Institute of Standards and Technology

\section{Objective}

To develop a system to quantify the level of accuracy of computer-based predictive fire models and the experimental, statistical, and analytical techniques to support this method.

\section{Problem}

Fire models have progressed to the point of providing good predictions for some parameters of fire behavior. However, users and authorities are often asked to accept building fire safety design based on fire models without accepted statements of their predictive accuracy. It is important to be able to state, with confidence, how close are the actual conditions to those predicted by the model. There is no domestic or international agreement on a procedure for quantifying fire model accuracy and demonstrating suitability for a particular application.

\section{Approach}

During FY 1994, BFRL will organize and coordinate domestic and international meetings to discuss and decide the suitability of computer-based predictive fire models as design tools. BFRL will try to reach agreement on a procedure for quantifying the accuracy and acceptability of these models. To facilitate this, BFRL researchers will develop appropriate procedures for ASTM consideration as an improvement over the current guide (ASTM E135590). Once developed, it is envisioned that numerous calculational tools and experimental techniques will be required to support the process. BFRL will work to develop such tools and techniques using its fire models to provide sample data for development of the tools. Two areas are identified for application during FY 1994: 1. development of experimental measurement techniques; and 2. examination of their variability to better understand the quality of future real-scale experiments designed to be used for model evaluation and examination of techniques for sensitivity analysis of complex fire models.
Recent Results

Peacock, R.D., Jones, W.W., and Bukowski, R.W., "Verification of a Model of Fire and Smoke Transport," Fire Safety Journal, 21, pp 89-129, 1993.

Matsushita, T. and Klote, J.H., Smoke Movement in a Corridor - Hybrid Model, Simple Model, and Comparison with Experiments, NISTIR 4982, National Instituta of Standards and Technology, Gaithersburg, MD, 1992.

Davis, W.D., Forney, G.P., and Klote, J.H., Field Modeling of Room Fires, NISTIR 4673, National Institute of Standards and Technology,

Gaithersburg, MD, 1991. 


\section{Development of the Fire Data Man- agement System (FDMS) Database System for Fire Related Data}

Principal Investigator:

Rebecca W. Portier

Fire Safety Engineering Division

301-975-6757

Sponsor:

National Institute of Standards and Technology

Objective

To develop a second generation database which accommodates all fire related data for users who collect or use building fire data.

\section{Problem}

A unified method of accessing data is crucial to experimental and modeling efforts to develop the science of fire. The FDMS concept is well founded, and very important to experimentalists acquiring data, to modelers and others using data related to fires and material properties. A stand-alone, PC version of the database exists which supports a limited number of fire test results. This version lacks some functionality, is not portable across computer platforms, and is dependent upon thirdparty software libraries which are no longer supported. These problems will be remedied by the second generation database.

\section{Approach}

This is the second year of a three year project. During FY 1994, BFRL will implement the file formats proposed in the NIST Technical Note 1407 (see below under Recent Results), importing existing data into the FDMS 2.0 database, and providing limited access to a test version of the database. This work will require reformatting numerous test data files from a variety of existing formats into a standard import format. The existing test data files include cone and furniture calorimeter, LIFT (Lateral Ignition and Flame Spread Test Apparatus), Eurific Corner tests, and some full-scale test data. A simple text-oriented user interface will be provided as a front-end for the test version of the database.

During FY 1995, BFRL will develop a graphical user interface which can be ported from multi-user to single-user platforms. This work will include import/export functionality, querying, and report writing capabilities. The results of this research are a centralized database of fire test values which will be accessible to modelers, experimentalists, practicing engineers, and others within the fire community.

\author{
Recent Results \\ Portier, Rebecca W., Fire Data Management Sys- \\ tem, FDMS 2.0, Technical Documentation, NIST TN \\ 1407, National Institute of Standards and Technolo- \\ gy, Gaithersburg, MD, 1994.
}

Portier, Rebecca W., A Programmer's Reference Guide to FDMS File Formats, NISTIR 5162, National Institute of Standards and Technology,

Gaithersburg, MD, April 1993.

Grants

"The Development of the Fire Data Management System," F. Mowrer, University of Maryland. 


\section{HAZARD Development}

Principal Investigator:

Walter W. Jones

Fire Safety Engineering Division

301-975-6879

\section{Sponsor:}

National Institute of Standards and Technology

Objective

To plan and implement the next generation hazard methodology.

\section{Problem}

Traditional approaches to product design, product evaluation, and codes and standards development address fire safety in a piecemeal fashion - for example, evaluating heat release without considering product use, toxicity, or ignition propensity. Quantitative hazard analysis techniques have the potential of providing significant cost savings. In addition, measures are evaluated as an interacting system, including the impact of both structure and contents. Alternative protection strategies can be studied within the hazard analysis framework to give the benefit-cost relation for each. Providing these alternatives promotes the design flexibility which reduces redundancies and cost without sacrificing safety.

\section{Approach}

The project is being performed as three tasks:

1. advancing the physics in the model,

2. improving the structure, interface and interaction of the individual components of the models of HAZARD 1, and

3. publishing the methodology.

During FY 1994, BFRL will test the single-surface pyrolysis, detection and suppression algorithms. The unification of the user interface with newgeneration input editor and hazard shell including a new unified input data file will continue. New physics of fire will be incorporated into the model such as compartment-to-compartment heat transfer through walls.

This methodology and associated software will enable architecture and engineering firms to demonstrate effectiveness of novel building design to code compliance authorities. It will also enable product manufacturers to test building components early in the design phase, prior to the costly steps of engineering and product development.

Recent Results

Continue maintained close cooperation with Worcester Polytechnic Institute to further their teaching this methodology of fire hazard analysis for fire protection engineers.

Initiate a CRADA with the Portland Cement Association to develop models for structural integrity and barrier penetration.

Portier, R.W., Reneke, P.A., Jones, W.W., and Peacock, R.D., A User's Guide for CFAST Version 1.6, NISTIR 4985, National Institute of Standards and Technology, Gaithersburg, MD, 1992.

Peacock, R.D., Jones, W.W., Bukowski, R.W., and Forney, C.L., The Fire Hazard Assessment Method, NIST Handbook 146, Volumes I and II, National Institute of Standards and Technology, Gaithersburg, MD, 1991.

Grants

"Expansion of the Applicability of EXIT89," Fahy, R.F., National Fire Protection Association.

"Development of a Graphical Interface for PostProcessing the Results of a CFAST Simulation," Barnett, J., Worcester Polytechnic Institute.

"Computational Heat Transfer for Zone Fire Modeling," Moss, W.F., Clemson University.

"A Survival (Egress and Tenability) Model for Hazard Modeling," Mowrer, F. and Weissman, B., University of Maryland.

"Mathematical Modeling of Human Egress from Fires in Residential Buildings," Kostreva, M., Clemson University. 


\section{Modeling of Detector Response}

Principal Investigator:

Walter Jones

Fire Safety Engineering Division

301-975-6887

Sponsor:

National Institute of Standards and Technology

Objective

To assist Rolf Jensen \& Associates, Inc. (RJA) evaluate the Harwell Flow3D code for the analysis of detector response in a specific building fire.

\section{Problem}

RJA needs to evaluate the expected performance of fire detectors under specific and highly complex conditions. The only means to perform the analysis is Computational Fluid Dynamics (CFD) models in combination with analysis techniques recently developed by BFRL in a project sponsored through the National Fire Protection (NFP) Research Foundation.

\section{Approach}

During FY 1994, BFRL with staff from RJA will analyze the Flow3D model (a commercial package computer model that simulates fluid dynamics and heat transfer using a finite element difference approach). BFRL will provide orientation, technical advice, and aid in model set-up and analysis. RJA will provide the data and information needed to perform the analysis including a detailed description of the fire, its growth, and time line, and drawings showing spatial information and other details. The results of this project are expected to demonstrated the full range of modeling tools, simple hand calculations, zone models such as CFAST (BFRL's building fire model) and sophisticated CFD models can be used in concert to address problems in understanding the course of a fire in complex buildings.

Recent Results

New project.

\section{Furniture Flammability}

\author{
Principal Investigator: \\ Walter Jones \\ Fire Safety Engineering Division \\ 301-975-6887
}

\section{Sponsor:}

National Institute of Standards and Technology

\section{Objective}

To develop guidelines for furniture manufacturers to design residential upholstered furniture with low rate of heat release.

\section{Problem}

Upholstered furniture remains the leading "first item ignited" in fatal residential fires. Furniture with low peak rate of heat release will, on its own, not result in flashover of most rooms and therefore not lead to fatalities. A number of states limit the rate of heat release of public occupancy furniture measured in the California TB 133 test to $80 \mathrm{~kW}$, a figure that would eliminate a large proportion of current residential furniture. The National Fire Protection Association committee on furnishings and contents is discussing a possible limitation in the region of $250 \mathrm{~kW}$ for residential furniture. Most manufacturers of residential furniture offer thousands of different fabrics and many furniture styles. New products are introduced every 6 months. Furthermore, most manufacturers do not have burn facilities or the experience in designing furniture to meet heat release limitations. Therefore it is important to provide guidance on cost effective steps that can be taken to meet specific limitations and minimize any full scale testing that might be required. Although many items of furniture have been tested by commercial laboratories, bench scale tests on the upholstery materials have not been performed in most cases. Prediction of heat release of various designs, especially low heat release designs, from bench scale data on materials is not good because of the unquantified influence of shape and factors that cause transition from smoldering or sudden delayed flaming flareup are not understood. Guidance on changes that may be made to improve full scale performance to meet the public occupancy requirement or other potential requirements is needed. 


\section{Approach}

During FY 1994, BFRL will complete work on interliner performance for predicting the heat release of residential furniture. Using mockups, BFRL will systematically examine the effect on heat release rate of design factors such as heat release and flame spread rate of the fabric, seat width, seat back height and angle, and arm height. Using information from these mockup burns and from the videotapes of full-scale tests a predictive model of the burn process will be developed. The model will address initial ignition pattern of various ignition sources including the TB133 burner, flame spread across the surfaces, burning rate of the furniture, and possible ignition of other items. The predictive model combined with a room fire model will calculate the impact of various sized ignition sources and various levels of flammability on residential furniture fires. Rules for designing low heat release furniture and calculation procedures for predicting mockup and full size item performance from Cone calorimeter and LIFT (Lateral Ignition and Flame Spread Test Apparatus) data will be developed. These rules and calculation procedures will be tested against commercially available furniture items. Because California TB133 has proved to be a useful test and an incentive for reducing total heat release, BFRL will participate in a round robin of an ASTM version of the California TB 133 test to address repeatability.

\section{Recent Results}

Ohlemiller, T.J. and Villa, K.M., "Characterization of the California Technical Bulletin 133 Ignition Source and a Comparable Gas Burner," Fire Safety Journal, 18, pp 325-354, 1992.

Cleary, T.G., Ohlemiller, T.J., and Villa, K.M., The Influence of Ignition Source on the Flaming Fire Hazard of Upholstered Furniture, NISTIR 4867, National Institute of Standards and Technology, Gaithersburg, MD, June 1992.

\section{Support of Fire Modeling on the ex-USS SHADWELL}

\author{
Principal Investigator: \\ Walter $W$. Jones \\ Fire Safety Engineering Division \\ 301-975-6879
}

Sponsor:

Naval Research Laboratory

Chemistry Division

\section{Objective}

To develop algorithms to enhance the predictive capabilities of CFAST (BFRL's building fire model) in handling semi-confined fire development applicable to Navy ships.

\section{Problem}

Fire growth models for ships are similar to but sufficiently different that building models. Additional phenomena must be incorporated in these models to solve problems specific to ship design. An example is the process of fire spread by conduction through decks.

\section{Approach}

During FY 1994, BFRL will begin development of an algorithm that calculates conductive heat transfer through walls to adjacent compartments with arbitrary connectivity and thermophysical properties. The ceiling/floor heat transfer development will be performed this year and the data structures for including heat transfer through adjacent walls will be planned. The Navy is particularly interested in the ceiling/floor aspect in solving the problems encountered in the STARK fire in the Persian Gulf. BFRL researchers will begin planning to incorporate horizontal flow into CFAST. This is the hybrid extension which is important for detector citing and for testing smoke mitigation efforts with pressurization. This work is expected to provide a tool to assess ship configuration which can be used in place of full scale testing, particularly for considering insulation on bulkheads and ceilings.

\section{Recent Results}

Developed an algorithm that calculates the conductive heat transfer through walls to adjacent compartments with arbitrary connectivity and thermophysical properties. 


\section{Computing \& Network Resources}

Principal Investigator:

James W. Raines

Fire Safety Engineering Division

301-975-6855

Sponsor:

National Institute of Standards and Technology

\section{Objective}

To provide improvements, day to day support, and long range planning for scientific and administrative computing.

\section{Problem}

Efficient computing requires a uniform and consistent environment for scientific and administrative services.
Approach
During FY 1994, BFRL will configure, purchase, and install new hardware and operating software for the local area networks to provide a uniform and consistent system. Provide system backup for management and support staff. Add new users to the LANs and upgrade software as necessary. Complete plans for a separate LAN for scientific use and install.
Recent Results
Increased the use of LAN and shared resources, computer downtime was kept to a minimum, and provided computer upgrades for staff members who need new equipment. 
FIRE SAFETY ENGINEERING DIVISION

\section{LARGE FIRE RESEARCH}




\section{Simulation of Turbulent Combus- tion and Transport in Fires}

Principal Investigator:

Howard R. Baum

Fire Safety Engineering Division

301-975-6668

\section{Sponsor:}

National Institute of Standards and Technology

\section{Objective}

To develop a fundamental understanding of the mechanisms which control the gas phase combustion and transport processes in fires and a predictive capability which will allow the computer simulation of these processes to be based on the mathematical expression of underlying physical principles.

\section{Problem}

Information is lacking about the interaction of a fire with its environment, whether that environment is an individual room in a building fire or the atmosphere in the vicinity of a large outdoor fire. The transport and deposition of smoke generated in crude oil fires is of interest to the Mineral Management Services, the State of Alaska, the U.S. Coast Guard, and the American Petroleum Institute. The interaction of fires with the building environment has been a major research topic for BFRL and its international counterparts. Small scale combustion processes are the study of diffusion flames in a turbulent flow environment and as such are of interest to the general combustion community.

\section{Approach}

Theoretical and computational techniques applied to the study of transport, mixing, diffusion, radiation, and reaction processes occurring at widely different length and time scales. Detailed studies of individual phenomena are carried out in a manner that permits them to be assessed individually and later combined into overall simulations of problems of interest.

\section{Recent Results}

Enclosure fire simulation code compared with salt water and reduced scale enclosure fire experiments at U.C. Berkeley and California Institute of Technology demonstrated excellent agreement for large scale convective transport in two dimensional scenarios.
Three-dimensional smoke plume model compared with experiments at Mobile, AL and used for environmental burning assessment for several organizations in the United States and abroad.

Grants

"Soot, Carbon Monoxide, and Radiant Emissions of Luminous Turbulent Flames," Faeth, G.M., University of Maryland.

"Development of a Computational Model for Large Fire Plumes," Ghoniem, A.F., Massachusetts Institute of Technology. 


\section{Development of Large Eddy Enclosure Fire Model}

\author{
Principal Investigator: \\ Howard R. Baum \\ Fire Safety Engineering Division \\ 301-975-6668
}

\section{Sponsor:}

National Institute of Standards and Technology

\section{Objective}

To develop a three dimensional large eddy simulation capability for heat and smoke movement in mechanically ventilated enclosed commercial and industrial spaces.

\section{Problem}

There is an internationally expressed need for an advanced heat and smoke transport simulation capability for fire scenarios in large mechanically controlled environments. Commercial Computational Fluid Dynamics (CFD) codes cannot offer the required spatial and temporal resolution due to their generality of purpose and turbulence models, while their proprietary nature prevents generally available modification. The present commercial codes depend upon empirical turbulence models for their output, inherently limiting their predictive capability. This restriction leads to an ongoing burden of detailed experimental verification of the computations for each new fire scenario or building geometry.

\section{Approach}

During FY 1994, BFRL will initiate the development of a code combining methodologies developed separately at Tokyo University for mechanical ventilation flows and at BFRL for fire induced smoke transport to produce code specialized for enclosure fire scenarios based on large eddy simulation techniques. BFRL will meet with Japanese colleagues to plan appropriate division of tasks and strategy for delivery of finished product. Initial validation experiments conducted using large mechanically ventilated test facility at the Fire Research Institute, Japan with BFRL field instrumentation capability and personnel. Additional validation experiments to be performed at enhanced BFRL large scale test facility.
The resulting code will offer an alternative to general purpose commercial computational fluid dynamics codes. The alternative code will be a high resolution product with a minimum of empiricism that is directly focused on fire problems in large enclosures.

\section{Recent Results}

New project.

\section{Grants}

"An Investigation of Oil and Gas Well Fires and

Flares," Gore, J.P., Purdue University.

"Large Fire Analysis," Pagni, P.J., University of California (Berkeley).

"Fire Protection in Large Industrial Fires," DiMarzo, M., University of Maryland. 


\section{Analysis of Large Industrial Fires}

\author{
Principal Investigator: \\ David Evans \\ Fire Safety Engineering Division \\ 301-975-6897
}

\section{Sponsor:}

National Institute of Standards and Technology

\section{Objective}

To develop engineering methods for analysis of large fire events in industrial facilities.

\section{Problem}

The present generation of BFRL fire models are best suited to analyze fire hazards in light industrial office occupancies or dwellings where only ordinary furnishing and building finish materials may be involved. In many cases these models cannot accurately address fire situations in industrial facilities. Many of the constructed facilities in use by U.S. industry are different than office occupancies and dwellings from the standpoint of design, fire spread, fire protection, fire fighting, and types of fuels. Analytical tools must be developed for U.S. industry so that fire events in industrial facilities can be analyzed to the same level of accuracy as that provided by fire models for office and residential buildings. It is necessary to determine from industry, the fire safety concerns for their facilities and to prioritize those needs. Problems that are likely to concern industry are potential ignition sources including arson, combustion product emissions, safe spacing for facilities, fire fighting, ground water and air pollution, and the failure of industrial structures exposed to fire. Industrial facilities include most engineered facilities used by the petro-chemical manufacturing, refining and storage industries, associated with the distribution system for industrial products, and unique industrial research and demonstration ventures.

\section{Approach}

During FY 1994, BFRL will seek input from practicing fire protection engineers to collect information about the needs of industry to increase the knowledge base of fire phenomena and analytical methods to assess fire hazards. The input will be organized to determine future research tasks and their priority. Concurrent with the information gathering and analysis, a study of urban fires resulting from earthquakes will be initiated to determine a role for BFRL in mitigating fire damage from natural disasters and in promoting rapid recovery of normal operation. Information from this study will be used as the basis for future projects that will contribute to specific industrial fire and disaster mitigation needs.

Recent Results

New project. 


\section{In Situ Burning of Oil Spills}

Principal Investigator:

David Evans

Fire Safety Engineering Division

301-975-6897

\section{Sponsor:}

Department of Interior

Minerals Management Service

Technology Assessment and Research Branch

\section{Objective}

To conduct laboratory and mesoscale experiments to investigate the burning of weathered and emulsified crude oils on water.

\section{Problem}

BFRL has established leadership in characterizing the combustion of large pool fires. In support of other agency objectives to implement burning as a response method to oil spills, the burning characteristics of fresh, weathered and emulsified oils must be known. In addition, predictions are needed to assess the impact of the burning on downwind locations. BFRL is providing this information through its measurement and analysis effort.

\section{Approach}

During FY 1994, BFRL will organize data from previous laboratory and mesoscale experiments to support applications for permits to perform mesoscale tests in a wave tank. A conference/workshop will be conducted, similar to the 1988 conference to assess user needs. Measurements on the ignition and burning characteristics of weathered and emulsified crude oils will be made in controlled laboratory experiments. Eventually, results of these studies will be organized into a practical guide for responders to know under what conditions burning can be successfully applied to spills. The Large Eddy Simulation (LES) model for smoke plume flow in the atmosphere will be modified to account for non-steady burning conditions. This research supports the establishment of in-situ burning as an oil spill response method and the development of state and local requirements for its use in an actual oil spill emergency.

\section{Recent Results}

Walton, William D., In Situ Burning of Oil Spills:

Mesoscale Experiments and Analysis, NISTIR 5192,
National Institute of Standards and Technology, Gaithersburg, MD, September 1993.

Walton, W.D., Evans, D.D., McGrattan, K.B., Baum, H.R., Twilley, W.H., Madrzykowski, D., Putorti, A.D., Rehm, R.G., Koseki, H., Tennyson, E.J., "In Situ Burning of Oil Spills: Mesoscale Experiments and Analysis," Proceedings of the Sixteenth Arctic and Marine Oil Spill Program Technical Seminar, v2, pp 679-734, Edmonton, Alberta, Canada, Environment Canada, Ottawa, Ontario, June 7-9, 1993.

Notarianni, K.A., Evans, D.D., Walton, W.D., "Smoke Production from Large Oil Pool Fires," Proceedings Interflam '93, Fire Safety, Sixth International Fire Conference, March 30-April 1, 1993, Oxford, England, Interscience Communications, Ltd., London, England, Franks, C.A., ed., pp 111-119, 1993. 


\section{Smoke Plume Sampling}

Principal Investigator:

William D. Walton

Fire Safety Engineering Division

301-975-6872

\section{Sponsor:}

U.S. Coast Guard

Research and Development Center

\section{Objective}

To design and test two smoke plume sampling packages, one that can be suspended from a helicopter and another that can be suspended from a miniblimp to monitor in situ crude oil burns.

\section{Problem}

Although laboratory and mesoscale test burns have demonstrated the effectiveness of in situ burning as an oil spill mitigation technique, questions remain about the composition of the smoke in the plume downwind of the fire. Measurements of the smoke composition are necessary to provide information for decision makers. To measure the composition of the smoke in the plume it is necessary to take samples over an extended period of time. The only means for maintaining a sampling package in the plume for an extended period of time appear to be helium filled miniblimps and helicopters. The blimps provide a good measurement platform near the fire but may not be capable of obtaining the altitude required to make downwind measurements. Helicopters can fill this need although at present a sampling package does not exist which can be suspended from a helicopter and held in the smoke plume.

\section{Approach}

BFRL has used smoke sampling packages suspended from miniblimps for several years during mesoscale burns in Norfolk, Virginia and Mobile, Alabama. The design of these packages has been improved several times to reduce the weight and increase the reliability. Improvements to the package design have been developed after the most recent mesoscale burns and a new package will be constructed for use in burns of opportunity. The miniblimp transported smoke sampling package would contain air sampling pumps, collection media, and sample bags to provide time averaged measurements of smoke yield, smoke particle size distribu- tion, PAH concentration, and volatile organic compounds.

During FY 1994, BFRL will purchase a new miniblimp and a new portable winch for the blimp line will be constructed which can be transported as luggage on commercial airline flights. Also, BFRL will develop a helicopter transported instrumentation package. The helicopter transported instrumentation package, about the size of a suit case, would contain air sampling pumps, collection media, and sample bags to provide time averaged measurements of smoke yield, smoke particle size distribution, $\mathrm{PAH}$ concentration, and volatile organic compounds. In addition, the package would contain basic metrology measurement of temperature, relative humidity, wind speed and direction, and barometric pressure. The package would include the capability to turn all of the sampling pumps on and off by remote control and would contain a strobe light to indicate that the pumps were operating. The package would be designed to be suspended from the winch on a Coast Guard HH-65A helicopter that has a cable with a working length of $75 \mathrm{~m}$. The package would be held in a specific relative location in the plume for approximately 15 minutes in order to obtain an adequate quantity of sample for analysis. The prototype package will be tested for flight characteristics on a Coast Guard helicopter in Mobile, Alabama.

The helicopter and miniblimp transported packages will be evaluated during three mesoscale fuel oil burns at the Fire and Safety Test Detachment in Mobile, Alabama. BFRL will provide analysis of the smoke yield and particle size distribution and analysis of the PAH compounds.

\section{Recent Results}

The development and testing of the smoke sampling packages has been successfully computed. A final report is in preparation. 


\section{Smoke Plume Trajectory from in situ Burning of Crude Oil in Alaska}

\section{Principal Investigator:}

Kevin B. McGrattan

Fire Safety Engineering Division

301-975-2712

\section{Sponsor:}

Alaska Department of Environmental Conservation

Division of Spill Prevention and Response

Objective

To determine the expected trajectory of smoke plumes from in situ burning as a response method for oil spills in Alaska.

\section{Problem}

There is a need to determine the downwind concentration of particulate matter from the in situ burning of crude oil in Alaska.

\section{Approach}

During FY 1994, BFRL will determine the downwind concentrations of smoke particulate from the in situ burning of oil on water. This work involves using the Large Eddy Simulation (LES) model of a smoke plume trajectory to address problems of interest to the Alaska Department of Environmental Conservation. This work has provided the State of Alaska with guidelines on how far downwind one might expect to find high levels of particulate matter from an in-situ burn of oil. These estimates will be used in the decision making process of whether to use insitu burning as a spill response method.

\section{Recent Results}

McGrattan, K.B., Putorti, A.D., Twilley, W.H., and Evans, D.D., Smoke Plume Trajectory from In Situ Burning of Crude Oil in Alaska, NISTIR 5273, National Institute of Standards and Technology, Gaithersburg, MD, October 1993.

\section{Office Building Fire Research Program}

\author{
Principal Investigator: \\ Daniel Madrzykowski \\ Fire Safety Engineering Division \\ 301-975-6677
}

\section{Sponsor:}

General Services Administration

Public Building Services

Office of Real Property Management and Safety

\section{Objective}

To quantify the impact of large fires on buildings and their occupants and investigate the use of current technology/resources for mitigating the hazards.

\section{Problem}

Systems furniture or "work stations" have been identified as a source of large Heat Release Rate (HRR) fires in office buildings. Because of the wide spread use of systems furniture the potential fire hazard in an open plan office environment needs to be quantified. The conditions in a long corridor (means of egress) adjoining the fire compartment need to be determined. If the fire can not be suppressed in the room of origin, the impact of sprinklers on the smoke flow in the corridor is needed. While the impact of sprinklers has been demonstrated in experiments at BFRL, this has not been included in the corridor flow model, because the existing data base and zone modeling techniques are not sufficient to continue development of the corridor flow model including sprinkler effects.

\section{Approach During FY 1994, BFRL will investigate the extent that materials or geometry effect the fire perfor- mance of systems furniture. A combination of laboratory scale and full scale tests will be used to quantify the fire development and address means of mitigating the potential fire hazard. Multiple work station fire tests will be conducted in a large compartment. The compartment will have one wall open to simulate the effects of a typical open plan office space.}

The USCG's corridor fire test facility will be used to measure smoke flows down a corridor with and without sprinkler intervention. Results from these 
tests will be compared with predictions from the existing corridor model and the Harwell FLOW 3D la commercial computer model that simulates fluid dynamics and heat transfer using finite difference approach) field model.

The results of this work will provide a technical basis for assessing the hazards associated with work stations in an open plan office environment and smoke flow in corridors.

\section{Recent Results}

New project.

\section{Large Fire Test Facility}

Principal Investigator:

Emil Braun

Fire Safety Engineering Division

301-975-6665

Sponsor:

National Institute of Standards and Technology

\section{Objective}

To perform large scale fire tests and provide data to support other fire research projects and to apply, refine, and develop methods and equipment that contribute to the successful conduct of large scale fire tests.

Problem

Use of BFRL's Large Scale Test Facility (Building 205) must be scheduled for timely performance of tests. The size of the facility and capacity of its afterburner system has placed severe limits on type, number, and scope of tests that can be performed.

\begin{abstract}
Approach
During FY 1994, BFRL will continue to provide daily management of the Large Fire Test Facility, which includes scheduling tests and timely execution, along with gradual modernization of test equipment. Additional short term improvements will be developed and executed during the fiscal year, pending funding availability. These plans will be developed in conjunction with researchers to assure that new and updated instruments fit the needs of the fire program.
\end{abstract}

Work includes ensuring that BFRL's technicians, assigned to this test facility, are trained in the operation of all aspects of the building's facilities. The Cone Calorimeter will be brought online. New data acquisition software for the Cone Calorimeter, that is compatible with BFRL's other Cone Calorimeter, will be developed. Develop an upgraded plan of current facility that will allow for an expanded fire research program to include industrial commercial fire concerns as well as continue our efforts in the residential area.

\section{Recent Results}

Numerous tests have been performed including the 2/5 model, pool burns, large scale calorimeter tests (furniture calorimeter and main hood). 


\section{Cone/LIFT/Toxicity Support}

Principal Investigator:

Andrew J. Fowell

Fire Safety Engineering Division

301-975-6865

\section{Sponsor:}

National Institute of Standards and Technology

\section{Objective}

To provide technical support for standards activities associated with the Cone Calorimeter, Lateral Ignition and Flame Spread Test Apparatus (LIFT), and BFRL's development of the NIBS Toxicity Test.

\section{Problem}

As developer of the Cone Calorimeter, LIFT, and NIBS toxicity protocol, and promoter of bench-scale measurements as methods to provide input to predictive fire models, BFRL will continue to provide answers to technical enquiries by new users of the tests. Technical questions often arise at ASTM and ISO standards meetings, and at technical meetings addressing material flammability measurement. A program is underway in the furniture industry to obtain correlation between the Cone and the California TB 133 test. A key to improving the correlation is an improvement in the consistency of construction of the Cone test sample. A major furniture flammability program is underway in Europe in which the Cone Calorimeter is considered as a potential bench scale test as a screen for large scale tests. Recent in-house experiments using the Cone have revealed problems with calibration at high rates of heat release. These problems need to be resolved. A round robin performed on the LIFT test revealed some shortcomings especially in the preheat conditions used for flame spread measurements.

\section{Approach}

During FY 1994, BFRL will attend ASTM E.5 committee meetings to propose necessary changes to the protocols and ensure that changes made are technically correct. BFRL will work with other users of the LIFT apparatus to develop necessary changes to the ASTM procedure. Problems with the Cone calibration will be investigated and any needed modifications to the protocol will be proposed to ASTM.
Recent Results

ASTM and ISO have published standards for the BFRL Cone and the LIFT apparatus: ASTM E135492 Standard Test Method for Heat and Visible Smoke Release for Materials and Products Using the Oxygen Consumption Calorimeter and ASTM E1321-90 Standard Test Method for Determining Material Ignition and Flame Spread Properties respectively.

The BFRL developed NIST toxicity test is progressing through the ASTM and National Fire Protection Association (NFPA) standards process.

An international symposium on Flammability of Contents and Furnishings of Buildings was organized and the papers published as ASTM STP 1233, American Society of Testing and Materials, 1994.

Fowell, A.J., Interlaboratory Test Program of ASTM Standard E1321, Test Method for Determining Material Ignition and Flame Spread Properties, ISR07, PCN: 33-000007-31, American Institute for Standards Research, 1993.

Fowell, A.J., Developments Needed to Expand the Role of Fire Modeling in Material Fire Hazard Assessment, International Conference for the Promotion of Advanced Fire Resistant Aircraft Interior Materials," Federal Aviation Administration, DoT, February 1993. 


\section{Development of a Sizing Standard for Firefighter Protective Clothing}

Principal Investigator:

James R. Lawson

Fire Safety Engineering Division

301-975-6676

\section{Sponsor:}

Federal Emergency Management Agency

U.S. Fire Administration

Firefighter Health and Safety

\section{Objective}

To determine the critical factors of station/work garment size which affect the thermal protection and work dynamics of fire fighters during fire suppression operations.

\section{Problem}

As a result of recent changes in employment regulations in the fire service the range of firefighter body sizes has increased. The fit of firefighter station/work uniforms has become more critical as it relates to function with and without protective garments in fire suppression operations. Therefore, a need exists for standardizing the size of station/work uniforms to enhance safety and efficiency in fire fighting activities.

\section{Approach}

During FY 1994, BFRL will perform a literature survey to identify and obtain publications on the function of firefighter station/work uniforms. BFRL will obtain information from the fire services and apparel manufacturers to identify special needs of firefighters and the ability of industry to support the firefighter's needs. Working with ASTM and NFPA committees which produce standards related to firefighter protective garments, BFRL to develop and establish standards for firefighter station/work uniforms. These standards are expected to improve firefighters' safety by providing standard methods for determining proper sizing of station/work uniforms which should lead to better mobility and reduced fatigue.

\section{Recent Results}

- Lawson, James R. and McCarthy, Robert T., Statement of Need for a Standard Practice for Sizing of Fire and Rescue Services Station/Work Uniforms, submitted to ASTM Committee F-23 on Protective Clothing, June 1993.

ASTM Committee F-23 has authorized development of the new Sizing Standard for Station/Work Uniforms, June 1993. 
FIRE SCIENCE

DIVISION

\section{SMOKE DYNAMICS RESEARCH}




\section{Carbon Monoxide Production and Prediction}

Principal Investigator:

William M. Pitts

Fire Science Division

301-975-6486

\section{Sponsor:}

National Institute of Standards and Technology

\section{Objective}

To develop a fundamental understanding of the mechanisms of carbon monoxide (CO) formation in flames sufficient to produce an estimation and a detailed predictive model.

\section{Problem}

$\mathrm{CO}$ generated by fires in enclosures is responsible for roughly two-thirds of fire deaths. The conditions necessary and the mechanisms responsible for the generation of high concentrations of $\mathrm{CO}$ are poorly characterized. As a result, at present it is not possible to predict the generation of $\mathrm{CO}$ by fires. Such a predictive capability is required by Laboratory fire models such as Hazard 1 (BFRL's software that predicts the hazard to a building and occupants anywhere within a building).

\section{Approach}

During FY 1994, BFRL will continue to develop methods that predict $\mathrm{CO}$ in fires. In this work, BFRL is developing methods that correlate $\mathrm{CO}$ formation for fires in enclosures and providing knowledge to incorporate these findings into existing BFRL fire models. The fundamental investigations are focusing on identifying the principal chemical and physical mechanisms responsible for the formation of high $\mathrm{CO}$ concentrations in fires and to theoretically justify the use of the engineering correlations. Ultimately, the findings will be incorporated into a simple physical model for $\mathrm{CO}$ production in enclosure fires.

\section{Recent Results}

Archival manuscript on air flow into enclosure completed.

Pitts, W.M., Bryner, N.P., and Johnsson, E.L., "Production Mechanisms for Carbon Monoxide in Enclosure Fires," Combustion Institute Central and Eastern States Section, Combustion Fundamenta/s and Applications, pp 102-105, New Orleans, LA, March 13-17, 1993.

\section{Grants}

"Compartment Fire Combustion Dynamics," U. Vandsburger, Virginia Polytechnic Institute and State University and R.J. Roby, Hughes Associates. 


\section{Study of Smoke Agglomerates}

Principal Investigator:

George W. Mulholland

Fire Science Division

301-975-6695

\section{Sponsor:}

National Aeronautics and Space Administration

Exobiology Flight Experiments Program

\section{Objective}

To test the validity and utility of fractal concepts in describing the growth and properties of large smoke agglomerates.

\section{Problem}

Used is a general hardware design and data analysis methodology to study smoke agglomerates in an orbiter facility.

\section{Approach}

During FY 1994, BFRL will make use of Transmission Cell-Reciprocal Nephelometer recently developed at BFRL to monitor the extinction cross section and total scattering cross section as the agglomerates grow. The angle dependent scattering will be used to monitor the fractal dimension and average radius of gyration as the agglomerates grow. The results will be compared with light scattering calculations for a computer simulation of smoke agglomeration.

The results of this work will be used to develop design guidelines for optical diagnostics to monitor the structure and optical properties of soot during a microgravity experiment.

\section{Recent Results}

Mulholland, G.W., Bohren, C.F., and Fuller, K.A., "Light Scattering by Agglomerates: Coupled Electric and Magnetic Dipole Method," Langmuir (submitted for publication).

\section{Species Production for Turbulent Flame Modelling}

Principal Investigator:

Kermit C. Smyth

Fire Science Division

301-975-6490

Sponsor:

National Institute of Standards and Technology

Objective

To develop an accurate data base and phenomenological descriptions to establish models for in-flame species concentrations and post-flame species yields as a function of residence time, equivalence ratio, temperature, and strain and scalar dissipation rates.

\section{Problem}

The data base, new metrology, phenomenology, and models for well controlled flame environments will provide the foundation for predicting flame radiation and the production of toxic gases and smoke for buoyant, turbulent diffusion flames for a wide range of combustion conditions.

\section{Approach}

During FY 1994, BFRL will experimentally examine laminar flame environments with temperature, residence time, equivalence ratio, and strain and scalar dissipation rates characteristic of buoyant, turbulent flames. Emphasis will be placed on planar imaging approaches using laser-induced fluorescence and scattering signatures. Initial studies will focus on time-varying and underventilated laminar diffusion flames. Research will center on:

1. Characterizing the in-flame and post-flame chemical species, flow velocity, and temperature fields.

2. Applying GC-MS and laser ionization-mass spectroscopy (previously used for graphitic smoke) to the analysis of the mostly organic smoke produced during underventilated combustion.

3. Developing accurate diagnostic capability for soot volume fraction using a combination of optical and gravimetric techniques.

4. Testing and developing models that incorporate the wide range of combustion conditions using the new database. 
The results of this work will critically test the applicability of models for soot and species production to complex flowfields, i.e., practical fires.

\section{Recent Results}

Shaddix, C.R., Harrington, J.E., and Smyth, K.C., "Quantitative Measurements of Enhanced Soot Production in Flickering Methane/Air Diffusion Flame," Twenty-Fifth Symposium (Internationall on Combustion (submitted for publication).

Puri, R., Santoro, R.J., and Smyth, K.C., "The Oxidation of Soot and Carbon Monoxide in Hydrocarbon Diffusion Flames," Combustion and Flame, 97, 125, 1994.

Smyth, K.C., Harrington, J.E., Johnsson, E.L., and Pitts, W.M., "Greatly Enhanced Soot Scattering in Flickering $\mathrm{CH}_{4} /$ Air Diffusion Flames," Combustion and Flame, 95, 229, 1993.

Harrington, J.E. and Smyth, K.C., "Laser-Induced Fluorescence Measurements of Formaldehyde in a Methane/Air Diffusion Flame," Chemical Physics Letters, 202, 196, 1993.

Norton, T.S., Smyth, K.C., Miller, J.H., and Smooke, M.D., "Comparison of Experimental and Computed Species Concentration and Temperature Profiles in a Laminar, Methane/Air Diffusion Flame," Combustion Science and Technology, 90, 1, 1993.

\section{Short-Duration Autoignition Tem- peratures of Liquid Hydrocarbon Fuels}

\author{
Principal Investigator: \\ Nelson Bryner \\ Fire Science Division \\ 301-975-6868
}

\section{Sponsor:}

Department of the Air Force

Engineering and Services Laboratory

Objective

To investigate short-duration autoignition temperatures of liquid hydrocarbon fuels by extending previous autoignition experiments.

\section{Problem}

Fuels ignite on hot exterior surfaces of engines and turbines. Improved understanding of autoignition process is needed to formulate appropriate strategies for reducing autoignition tendencies, especially where fuel selection is concerned. The autoignition of hydrocarbon fuels on hot surfaces is a process of practical significance which occurs when selfsustained combustion of a gas mixture is initiated. Possible prevention can be accomplished by choosing fuels of the proper molecular structure, by specifying the composition, coatings, or treatment of the hot surface, or by other intervention strategies.

\section{Approach}

During FY 1994, BFRL will investigate the performance of different hydrocarbon fuel mixtures. Five pairs of differently structured fuels with the same number of carbon atoms will be compared. Experiments will use single ignition surface type and uniform mixtures of fuel to air. Most of the experiments will be conducted at ESL Tyndall Air Force Base using their Short-Duration Autoignition Apparatus.

The results of this research will assist the USAF Engineering and Services Laboratory increase their ability to formulate strategies to reduce autoignition tendencies where fuel selection is concerned. 


\section{Recent Results}

Developed procedure to evaluate short-duration autoignition temperatures for different hydrocarbon fuel mixtures. 
FIRE SCIENCE

DIVISION

\section{MATERIALS FIRE RESEARCH}




\section{Polymer Flammability Modeling}

Principal Investigator:

Marc R. Nyden

Fire Science Division

301-975-6692

\section{Sponsor:}

National Institute of Standards and Technology

\section{Objective}

To develop the technical basis for the design of a new generation of fire retardants and fire resistant materials.

\section{Problem}

Flammability is a major concern when polymeric materials are used in buildings, aircraft, ships, and clothing. Today's standards for acceptable cost, performance, and environmental safety have made the traditional "trial and error" approach to materials flammability obsolete. There is a strong correlation between char residue and fire resistance since char is always formed at the expense of volatile fuel. Furthermore, the presence of a surface char insulates unburnt material from the heat generated in gas phase combustion reactions and obstructs the outward flow of combustible gases generated in the degradation of the interior. The thermal degradation chemistry of some polymers can be altered to favor char formation with chemical additives and/or by direct modification of the structure of the polymer. The challenge is to achieve significant levels of fire resistance without adversely affecting intended-use properties, cost, and the toxicity of these materials.

\section{Approach}

During FY 1994, BFRL will continue developing its molecular dynamics model of the thermal degradation of polymers. The dynamic trajectories of the polymers are calculated from Hamilton's equations of motion. Macromolecular systems, which are too costly or too complex to synthesize on a routine basis, can be modeled and systematically varied, all the while examining their tendency to form high molecular weight crosslinked structures.

The manufacturers and the end users of polymer products will benefit from use of this new technology. They will have enhanced computer model applicable to a wider range of materials.

\section{Recent Results}

Nyden, Marc R., Forney, Glenn P., and Brown, James E., "Molecular Modeling of Polymer Flammability: Application to the Design of Flame Resistant Polyethylene," Macromolecules, 25, 1658, 1992.

Nyden, Marc R., Brown, J.G., and Lomakin, S.M., "Molecular Dynamics Modeling of Polymer Flammability," Materials Research Soc. Symp. Proc., 278, 47, 1992.

Nyden, Marc R. and Noid, Donald, W., "Molecular Dynamics of Initial Events in the Thermal Degradation of Polymers," J. Phys. Chem., 95, 940, 1991. 


\section{Burning Rate of Materials}

\author{
Principal Investigator: \\ Takashi Kashiwagi \\ Fire Science Division \\ 301-975-6699
}

\section{Sponsor:}

National Institute of Standards and Technology

\section{Objective}

To improve the understanding of the physical and chemical gasification processes of thermoplastics and char forming polymers and to develop theoretical models to predict the gasification rates of polymers exposed to fire conditions; to improve understanding of energy feedback mechanisms of pool fires and to develop theoretical models to predict energy feedback rates from a pool flame to the fuel surface; and to improve mathematical models to predict the burning rate of thermoplastics and to develop models for char forming polymers by combining the gasification models with the energy feedback rate models.

\section{Problem}

At present, mathematical models have not been developed that can calculate the burning rate (heat release rate) of building contents. Therefore, a fire source is prescribed or estimated from bench scale tests for HAZARD I (BFRL's software that predicts the hazard to a building and occupants anywhere within a building). To improve the prediction of fire hazard, accurate description of fire source is urgently needed.

\section{Approach}

During FY 1994, BFRL will develop burning rate models (global and field equation) of polymeric materials (including char formers) for simple horizontal configurations. This work is being performed as two parts:

1. Determining energy feedback rates from a pool flame for various fuels and sizes.

2. Determining the gasification rate of various polymers.

Gas phase combustion characteristics are measured for a variety of fuels with pool diameters up to 100 $\mathrm{cm}$. Global and field equation energy feedback models are developed as a function of fuel type and size. In the condensed phase, a well-defined thermal radiation source is used to simulate energy feedback from a flame to a polymer surface; the gasification rate, temperature and density distributions in the polymer are measured under nonflaming condition for various radiant fluxes. Gasification models for thermoplastics and char forming polymers are developed.

\section{Recent Results}

Choi, M., Hamins, A., Mulholland, G., and Kashiwagi, T., "Simultaneous Optical Measurement of Soot Volume Fraction and Temperature," Combustion and Flame (submitted for publication).

Hamins, A., Klassen, M., Gore, J., Fischer, S., and Kashiwagi, T., "Heat Feedback to the Fuel Surface in Pool Fires," Combustion Science and Technology (submitted for publication).

Yang, J., Hamins, A., and Kashiwagi, T., "Estimate of the Effect of Scale on Radiative Heat Loss Fraction and Combustion Efficiency," Combustion Science and Technology, 96, 183, 1994.

\section{Grants}

"Modeling of Combustion, Fluid Mechanics, and Radiation in Buoyant Turbulent Fires," O. Ezekoye, University of Texas.

"Fire Growth Models for Materials," J. Quintiere, University of Maryland.

"A Study of Entrainment and Flow Patterns in Pool Fires Using Particle Imaging Velocimetry," J. Gore, Purdue University. 


\section{Char Structure and Flammability}

\author{
Principal Investigator: \\ Takashi Kashiwagi \\ Fire Science Division \\ 301-975-6699
}

Sponsor:

National Institute of Standards and Technology

Objective

To characterize the chemical structure of polymer residues which are generated at well-defined fire simulated conditions.

\section{Problem}

Two recent trends which have started to affect the U.S. plastics industry are polymer recycling and a demand for nonhalogenated flame retardant treatments. The degradation products of polymers with such treatments are corrosive and some may be toxic. One alternative flame retardant approach is to form char during polymer burning. This approach is ideal because carbon atoms retained in the condensed phase as char lessen the available fuel for flaming combustion even as they help insulate the polymer from the heat of the flame. However, there is little quantitative information about the relation between polymer structure, char properties, and realistic flammability properties.

\section{Approach}

During FY 1994, BFRL will generate char samples from selected polymer samples in a heated flow tube at a variety of temperatures in an inert atmosphere. Char yield, $\mathrm{C} / \mathrm{H}$ ratio, aromatic/aliphatic ratio (NMR and FTIR), crosslinkage site density will be measured for the collected char for the selected samples. Gaseous degradation products for the selected polymer samples will be measured using a mass spectrometer. For higher heating rate conditions, thick $10 \times 10 \mathrm{~cm}$ block polymer samples will be exposed to external thermal radiant fluxes comparable to those in fire. These samples are heated in nitrogen, degraded, and partially charred without flaming in nitrogen. The exposed samples are sectioned into layers from the top of the exposed surface down to the unaltered polymer, yielding a series of snapshots of the thermal behavior of the polymer in fire-like conditions. The same measurements will be conducted for each layer of the samples. Heat transfer characteristics of the char residue are inferred by measuring the transient temperature of the backside of the sample. Flammability properties of the polymer materials are measured by bench-scale techniques.

The results of this work will serve as a guideline for development of new char forming flame retardants.

Recent Results

New project. 


\section{Flame Retardant Study on Engineering Plastics}

\author{
Principal Investigator: \\ Takashi Kashiwagi \\ Fire Science Division \\ 301-975-6699
}

\section{Sponsor: \\ General Electric Company \\ Chemical Research Center \\ Corporate Research and Development}

\section{Objective}

To establish and understand the effects of polymer structure and certain flame retardant treatments on flammability characteristics of specific engineering thermoplastics.

\section{Problem}

GE is interested in nonhalogenated flame retardant treatment to improve fire resistance performance of its engineering thermoplastics. Better flame retardant treatments are needed by understanding the chemical and physical nature of char and also with measurements of more meaningful flammability properties.

\section{Approach}

During FY 1994, BFRL will be measuring flammability properties of systematically varied polycarbonate and other engineering plastic samples including composite samples based on glass fibers. The effects of the structure of polycarbonate/siloxane copolymer samples on flammability properties will be determined. The effects of fiber variations and resins on flammability properties will be determined.

The results of this collaboration are expected to produce better flame retardant treatments by understanding the chemical and physical nature of char and flammability properties.

Recent Results

Kashiwagi, T. and Cleary, T., "Effects of Sample Mounting on Flammability Properties of Intumescent Polymers," Fire Safety J., 20, 203, 1993.

Kashiwagi, T., Cleary, T., Davis, G., and Lupinski, J., International Conference for the Promotion of Advanced Fire Resistant Aircraft Interior Materials and its paper was published in the Proceedings, DOT/FAA/93/3, FAA Technical Center, p175-187. 1993. 


\section{Flammability of Composite Materials}

Principal Investigator:

Thomas J. Ohlemiller

Fire Science Division

301-975-6481

James E. Brown

Fire Science Division

301-975-6483

Sponsor:

Department of the Navy

Naval Surface Weapons Center

\section{Objective}

To develop and validate a procedure, for using bench scale test results, that predicts full scale fire performance of composite materials.

\section{Problem}

Extensive Cone Calorimeter data on a variety of composite materials have been obtained in the past. The task remains to demonstrate that these data and parameters inferred from both these and from LIFT (Lateral Ignition and Flame Spread Test Apparatus) tests can be used to predict fire growth in compartments comprised of composite materials. The first challenge is to demonstrate an ability to predict upward flame spread performance on such materials. During the past 2 years, BFRL characterized this spread process in its radiant panel facility. An effort was started to test models developed by Cleary/Quintiere, Mitler, and Kulkarni against these data.

\section{Approach}

During FY 1994, BFRL will adapt the Cone Calorimeter, LIFT and other tests as needed to obtain input parameters for spread models and will compare these model predictions against spread data from radiant panel facility. In particular, attention will be given to pursuing the spread behavior of composites more typical of those likely to be used by the Navy. Experimental and modeling studies of flame retarded composites will be addressed during this year.

\section{Recent Results}

Measured flame spread behavior of two unretarded composites as a function of incident radiant flux and igniter size. 
Combustion of Silicone

Principal Investigator:

Takashi Kashiwagi

Fire Science Division

301-975-6699

Sponsor:

Dow Corning Company

\section{Objective}

To understand why the heat release rate las measured in the cone calorimeter) of burning siloxane is nearly independent of the external thermal radiant flux.

\section{Problem}

The heat release rate (HRR) of burning materials increases significantly with an increase in external thermal radiant flux applied to the burning surface. This is true for non-charring materials and also for charring materials. However, the burning behavior of siloxanes differs markedly from carbon-based materials. Perhaps most significant, the HRR for siloxanes (chain length $>15 \mathrm{~S}_{i}-\mathrm{O}_{2}$ units) does not increase significantly with an increase in external thermal radiant flux. Whereas carbon based materials may form products of incomplete combustion such as soot and carbonaceous chars, siloxanes form very minimal char but produce substantial amounts of solid amorphous silica $\left(\mathrm{S}_{i} \mathrm{O}_{2}\right)$ as a major product of combustion. Previous studies suggest that amorphous silica plays a significant role in mediating the burning behavior of siloxanes.

\section{Approach}

During FY 1994, BFRL will determine the flame radiative characteristics, energy feedback rate from flame to the sample surface, and general behavior of the sample in the condensed phase. One or more short chain length siloxanes and a much larger chain length siloxane will be used. The project consists of three parts:

1. characterization of the thermal radiation of siloxane pool flame vs. hydrocarbon pool flame (IR emission spectra and $x_{\theta}$ ),

2. measurement of the total energy feedback rates from flame at different external radiant fluxes, and 3. measurement of weight loss rate and the temperature distribution (depth profile) in the sample under different external fluxes.
Recent Results

New project. 


\section{Radiative Ignition and Subsequent Flame Spread Over Cellulosic Materials}

\author{
Principal Investigator: \\ Takashi Kashiwagi \\ Fire Science Division \\ 301-975-6699
}

\section{Sponsor:}

National Aeronautics and Space Administration

Lewis Research Center

Microgravity Science Program

\section{Objective}

To develop theoretical models of ignition and subsequent flame spread over a cellulosic material in a microgravity environment and calculate ignition and flame spread behavior using material characteristics determined in normal gravity.

\section{Problem}

This project is one of NASA Microgravity Science projects to enhance the understanding of combustion science by taking unique advantage of a microgravity environment.

\section{Approach}

During FY 1994, BFRL will be developing multidimensional (2D, axisymmetric and 3D) timedependent theoretical models of ignition and the subsequent transition to flame spread in a microgravity environment are developed and solved numerically. Two environmental conditions will continue to be studied; 1 . in a quiescent environment and 2. in slow forced flows. Validation experiments are planned for USML-2 flight in 1995. The conceptional experimental design, fabrication, experimental parameters and procedures are being developed working jointly with the NASA Lewis Research Center. Exploratory experiments with a similar hardware are being conducted using drop towers and $\mathrm{KC}-130$ flight.

The results of this research are expected to enhance fire safety in spacecrafts.

\section{Recent Results}

Yamashita, H., Baum, H., Kushida, G., Nakabe, K., and Kashiwagi, T., "Heat Transfer from Radiatively Heated Thick Material in a Low Reynolds Number
Microgravity Environment," J. Heat Transfer, 115, 418, 1993.

Kashiwagi, T. and Nambu, N., "Global Kinetic Constants for Thermal Oxidative Degradation of a Cellulosic Paper," Combustion and Flame, 88, 345, 1992.

Kushida, G., Baum, H., Kashiwagi, T., and diBlasi, C., "Heat and Mass Transport from Thermally Degrading Thin Cellulosic Materials in a Microgravity Environment," J. Heat Transfer, 114, 494, 1992. 


\section{Fire Resistant Aircraft Materials}

Principal Investigator:

Marc R. Nyden

Fire Science Division

301-975-6692

\section{Sponsor:}

Federal Aviation Administration

Aviation Safety Division

\section{Objective}

To assess the viability of using high energy irradiation to reduce the flammability of materials suitable for use in the interiors of commercial aircraft.

\section{Problem}

Synthetic polymers comprise a significant fraction of the fire load borne by commercial aircraft interiors. The flammability, smoke and toxicity characteristics of these materials may impact passenger survivability in the event of an in-flight fire or a post-crash fire. There is a strong correlation between char residue and fire resistance since char is always formed at the expense of volatile fuel. Surface char also insulates unburnt material from the heat generated in gas phase combustion reactions and obstructs the outward flow of combustible gases generated in the degradation of the interior. The thermal degradation chemistry of some polymers can be altered to favor the formation of a char with chemical additives and/or by direct modification of the structure of the polymer. The challenge is to achieve significant levels of fire resistance without adversely affecting intended-use properties, cost, and the toxicity of these materials.

\section{Approach}

During FY 1994, BFRL will use its molecular dynamics model of the thermal degradation of polymers to calculate the dynamic trajectories of the polymers from Hamilton's equations of motion. Macromolecular systems, which are too costly or too complex to synthesize on a routine basis, can be modeled and systematically varied while examining their tendency to form high molecular weight crosslinked structure.s.

\section{Recent Results}

Lomakin, S.M., Brown, James E., Breese, Robin S., and Nyden, Marc R., "An Investigation of the Thermal Stability and Char Forming Tendency of
Cross-linked Poly(methyl methacrylate)," Polym. Deg. and Stab., 41, 229, 1993.

Nyden, Marc R. and Brown, James E., "ComputerAided Molecular Design of Fire Resistant Aircraft Materials," Proceedings of the International Conference for the Promotion of Advanced Fire Resistant Aircraft Interior Materials, DoT/FA/93/3, 147, 1993. 
FIRE SCIENCE DIVISION

\section{FIRE SENSING AND EXTINGUISHMENT}




\section{Suppression of Simulated Engine Nacelle Fires}

\author{
Principal Investigator: \\ Anthony Hamins \\ Fire Science Division \\ 301-975-6598
}

\section{Sponsor:}

Department of the Air Force

Wright Laboratory

Survivability Enhancement Branch

Department of the Navy

Naval Air Systems Command

Department of the Army

Tank Automotive Command

Federal Avaiation Administration

FAA Technical Center

\section{Objective}

To document guidelines for fire suppression system performance based on improved understanding of the influence of various parameters on fire suppression in the engine nacelle.

\section{Problem}

An engine nacelle encases the jet engine compressor, combustor, and turbine. A nacelle fire is typically a turbulent diffusion flame stabilized behind an obstruction in a moderately high speed air flow. The most likely source for a fire in the nacelle are fuel lines carrying jet fuel or hydraulic fluid, that can feed the fire either as a spray or as a pre-vaporized gas. Temperatures as high as $150{ }^{\circ} \mathrm{C}$ are common in normal operating engine nacelles.

Extinguishment occurs when a critical amount of agent is transported to the flame, where it is entrained into the primary reaction zone. The extinction process is affected by a number of parameters, including the velocity of the air flow, the type and quantity of fuel, the system temperature, agent properties and concentration, and the flow field geometry (e.g., the location of obstacles in the flow field).

Halon $1301\left(\mathrm{CF}_{3} \mathrm{Br}\right)$ has been used as the fire extinguishing agent for protecting aircraft engine nacelles because of its many positive attributes.
However, its production will cease at the end of this year, leaving many aircraft needing an alternative. The Air Force soon will begin testing three candidate alternatives to halon 1301 in its full scale Engine Nacelle Test Facility. There are, however, a number of different aircraft and operating conditions for which a new suppressant is needed. Because testing cannot be performed for all possible aircraft and conditions, knowledge is needed which will provide guidance in the extension of the full-scale data to untested systems and conditions.

Recently, the Air Force funded an experimental study which involved simulating an idealized engine nacelle fire. A coaxial turbulent spray burner was used, with jet fuel and hydraulic fluid as the fuels with the air at ambient and elevated temperatures. This project will extend that study to a broader range of suppression and re-ignition conditions, typical of in-flight engine nacelles.

\section{Approach}

The project is grouped into six tasks; two will be completed in FY 1994 and the rest in FY 1995.

Task 1 is a review of engine nacelle geometries to understand better possible fire scenarios and to develop a test program.

Tasks 2 and 3 consist of flame suppression measurements which will be conducted in a modified turbulent spray burner. Task 2 will test the impact of increased agent $\left(\mathrm{CF}_{3} \mathrm{l}, \mathrm{HFC}-125, \mathrm{HFC}-227\right.$, and $\mathrm{N}_{2}$ ) temperature on flame stability. Task 3 will test the effect of flow parameters on the amount of agent $\left(\mathrm{CF}_{3} \mathrm{l}, \mathrm{HFC}-125, \mathrm{HFC}-227\right)$ needed to suppress a fuel-spray fire. The parameters to be tested in FY 1994 are the fuel flow, air flow, air temperature, and system pressure. The parameters to be tested in FY 1995 are the pre-burn time, the rate of application, and the flow field geometry (e.g., effect of an obstacle in the flow field).

Task 4 is a computational fluid dynamic model using FLOW3D (a commercial package computer model that simulates fluid dynamics and heat fransfer using a finite difference approach) of conditions in the engine nacelle. Information about criticality of the number, placement, and orientation of agent injection nozzles and the rate of gaseous agent injection will be determined. Task 5 will experimentally validate the flow field model developed in Task 4 and thereby demonstrate the capability of compu- 
tational fluid dynamics to model gaseous agent concentration in a generic engine nacelle.

Task 6 will compile the knowledge and data gained through Tasks 1-5 into guidance tools to assist suppression system designers. Information about concentration/duration requirements as a function of fire zone conditions will be included. Results will be used to give guidance for adjusting the needed concentration of candidate fire suppressants over a range of engine nacelle fire conditions.

Recent Results

New project.

\section{Risk Analysis for the Fire Safety of Airline Passengers}

\author{
Principal Investigator:
}

Richard L. Smith

Fire Science Division

301-975-6870

\section{Sponsor:}

Federal Aviation Administration

Technical Center

\section{Objective}

To develop a generic method and computer program that will compute the fire risk for airline passengers.

\section{Problem}

Airline fire safety is important for the airline passengers and for the potential flying public that needs to be assured of the air safety. The public has demonstrated a willingness to pay for flight safety when it is cost effective.

Injuries or deaths due to an airplane fire are a rare event. Therefore, there is not a large body of relevant statistical data that can be used to determine the impact of various fire safety strategies. In this respect, airplane fire safety is similar to the problem faced by the Nuclear Regulatory Commission for the safety of nuclear power plants or NASA for the safety of its space shuttle program.

\section{Approach}

In the last decade considerable progress has been made in developing and/or improving risk analysis. The major motivators were the Three Mile Island disaster and the Challenger space shuttle disaster. There have been major advances in Artificial Intelligence in dealing with reasoning based upon uncertainty or vague knowledge. Some advances have used such approaches as Bayesian Probability theory, belief networks, influence diagrams and new applications of the Maximum Entropy theory.

During FY 1994, BFRL will apply these advances to the risk analysis for the fire safety of airline passengers. BFRL will develop the generic methodology and a computer program that will enable the decision maker to calculate fire risk for airline passengers. The program will use the state-of-theart safety analysis technology, the historical data on fire losses in airplanes, the results of experiments 
and tests, the predictions of physical science models, and experts opinions. The decision maker will control what knowledge is used and what assumptions are made. The program will be capable of recording an individual analysis so that it can be reviewed by others.

\section{Recent Results}

Smith, Richard, Final Report for Risk Analysis for the Fire Safety of Airline Passengers, NISTIR, National Institute of Standards and Technology,

Gaithersburg, MD (in review).

\section{Advanced Fire Detection Systems}

\author{
Principal Investigator: \\ Richard Smith \\ Fire Science Division \\ 301-975-6870
}

Sponsor:

National Institute of Standards and Technology

\section{Objective}

To demonstrate to U.S. industry innovative techniques for sensing products of and precursors to a fire, to suggest standard fires for evaluating the performance of detectors for residential applications, and to demonstrate an approach to the intelligent processing of background non-fire signals.

\section{Problem}

A properly designed detection system must be able to identify in a matter of seconds a fire event which may occur only once in one hundred years, and the identification must lead to an action which is appropriate to the space being protected. This disparity in time scale and the variability in geometry, content and occupancy of the space impose great demands on the system. False alarms, maintenance problems, incomplete or inaccurate information, and inappropriate suppression responses are problems which can plague current fire detection systems, especially when cost is an overriding constraint. The phase out of halons and the increased capital investment in modern industrial processes make early sensing and suppression even more imperative.

\section{Approach}

During FY 1994, BFRL will work with representatives from the fire detector systems industry to better understand their needs and to transfer BFRL's results directly to its customers. Current fire detector test procedures will be reviewed to evaluate detectors and a methodology will be developed to characterize fires for use in evaluating detectors. The feasibility of describing the detectable parameters of fire ignition, as a function of time, will be investigated using multiple temperature readings on an occupancy for various time intervals. A computer program will be developed that will take this data in real time and determine the limit of fire detectability of this system. 


\section{Recent Results}

Grosshandler, W. and Braun, E., "Early Detection of Room Fires Through Acoustic Emission," 4th International Symposium on Fire Safety Science, Ottawa, Canada, June 1994.

Grosshandler, W. and Jackson, M., "Acoustic Emission of Structural Materials Exposed to Open Flames," Fire Safety Journal, 22, pp 209-228, 1994.

Grosshandler, W., RADCAL: A Narrow-band Model for Radiation Calculations in a Combustion Environment, NIST Technical Note 1402, National Institute of Standards and Technology, Gaithersburg, MD, April 1993.

\section{Grants}

"Development of an Economical Video Based Fire Detection and Location System," O.A. Plumb and R.F. Richards, Washington State University.

"Smart Fire Detection Using Neural Networks," J. Milke and T. McAvoy, University of Maryland.

\section{Protection of Data Processing Equipment with Water Sprays}

\author{
Principal Investigator: \\ William L. Grosshandler \\ Fire Science Division \\ 301-975-2310
}

Sponsor:

Federal Emergency Management Agency

U.S. Fire Administration

Objective

To determine the ability of a water spray to protect data processing equipment from the threat of a fire.

\section{Problem}

A total ban on halon production was implemented in January, 1994. There is a need to find suitable replacement fire suppressants. A water spray system is an attractive candidate for replacing halon 1301 in the protection of data processing equipment because water is an effective fire suppressant and is non-toxic, environmentally friendly, and competitively priced. The dynamics controlling the transport of the liquid water droplets are different from the dynamics of prevaporized halon 1301; hence, the problem is to determine the applicability of a liquid spray system in an environment currently protected with a gaseous agent.

\section{Approach}

During FY 1994, BFRL will contact the data processing industry, spray systems manufacturers, Underwriters Laboratory, and Factory Mutual to determine the environments in which mist systems are most likely to be considered. A representative data processing cabinet and room geometry will be defined and a likely ignition source and fuel load will be identified. A test facility will be modified to emulate the physical parameters thought to control the actual fire suppression process and baseline studies will be performed to determine the amount of a gaseous agent required to extinguish the test fire. The influence on extinguishing efficiency of the nozzle geometry, location relative to the fire, water application rate, and the depth to which the fire is buried within the simulated cabinet are parameters which will be examined. An experimental facility in which the droplet size distribution, velocity and obstructions to the flow can be independently controlled will be developed. 
Details of the fire test facility, hardware layout, and nozzle design will be provided to enable others to establish guidelines for the construction of a fullheight facility to test specific computer modules and spray hardware.

The results of this research will benefit those who are considering fine water sprays as an alternative to a gaseous replacement for halon 13011 in fire suppression systems.

\section{Recent Results New project.}

\section{Agent/System Compatibility for Halon 1301 Aviation Replacement}

\author{
Principal Investigator: \\ Richard G. Gann \\ Fire Science Division \\ 301-975-6866
}

\author{
Sponsor: \\ Department of the Air Force \\ Wright-Patterson AFB \\ Survivability Enhancement Branch \\ Department of the Army \\ Tank Automotive Command \\ Department of the Navy \\ Naval Air Systems Command \\ Federal Aviation Administration \\ FAA Technical Center
}

\section{Objective}

To provide data for appraising twelve USAF-specified candidate halon 1301 replacements for compatibility with flight systems, people, and the environment; recommend three candidates for further examination; and perform longer-term testing to increase confidence in the preliminary results.

Problem

The current suppressant for in-flight fires, halon 1301 , is rapidly being phased out. The Air Force has committed to begin implementing an alternative at the end of FY 1995. Screening of potential alternatives is needed to narrow the number of chemicals for full-scale testing.

\section{Approach}

During FY 1994, BFRL is working with researchers from NIST's Material Science and Engineering Laboratory in performing three tasks:

1. evaluating stability during long-term storage;

2. determining compatibility with three metals, six elastomers, and three lubricants;

3. developing prediction method for HF formation during suppression;

The results of this work will establish methods to evaluate future candidate suppressants while providing the best assessment of current conditions. 
Recent Results

Grosshandler, W.L., Gann, R.G., and Pitts, W.M., Evaluation of Alternative In-Flight Suppressants for Full-Scale Testing in Simulated Aircraft Engine Nacelles and Dry Bays, NIST Special Publication 861, National Institute of Standards and Technology, Gaithersburg, MD, April 1994.

\section{Agent Screening for Halon 1301 Aviation Replacement}

Principal Investigator:

William L. Grosshandler

Fire Science Division

301-975-2310

\section{Sponsor:}

Department of the Air Force

Wright Laboratory

\section{Objective}

To publish the findings on BFRL's experimental program developed during FY 1992 and 1993 for screening 11 gaseous agents, sodium bicarbonate powder, and other promising chemicals as a means to identify the best candidates for a subsequent fullscale aircraft fire extinguishment evaluation program.

Problem

An increased understanding of the key issues which control extinguishment in aircraft engine nacelle and dry bay fires has been gained through a multi-year experimental program. BFRL identified four agents for the Air Force to test in its full-scale facility during FY 1994.

\section{Approach}

During FY 1994, BFRL will document the various aspects of this research. This data will be published as a NIST Special Publication.

\section{Recent Results}

Developed a list of agents recommended for fullscale testing and a description of the rationale used to arrive at the recommendation. 


\section{Dynamics of Fire Suppression}

\author{
Principal Investigator: \\ Gregory T. Linteris \\ Fire Science Division \\ 301-975-2283
}

\section{Sponsor:}

National Institute of Standards and Technology

Department of the Air Force

Wright Laboratory

Survivability Enhancement Branch

Department of the Army

Tank Automotive Command

\section{Objective}

To develop a fundamental understanding of the mechanisms of flame extinction by existing fire suppressants and to enable the development of the next generation of suppression agents and technologies for the fire suppression industry.

\section{Problem}

Fire suppressants and their application methods need be effective, fast-acting, economical, have low toxicity, permit a habitable environment during suppression, leave no residue, and cause no damage to the protected space. Existing suppressants, however, are non-ideal because water can cause excessive damage, metal-based suppressants leave a residue, and the currently favored suppressants, the halons, may leave a corrosive residue after passing through a flame. Also, because of their suspected destruction of stratospheric ozone, the most effective halons are being phased out. There exists a continuing need for better suppressants and suppression methods.

\section{Approach}

During FY 1994, BFRL is developing an understanding of the mechanisms of existing fire suppressants by designing laboratory-scale experiments, performed in conjunction with chemical kinetic calculations of the temperature and species concentration profiles in inhibited flames. The measurements include global properties of inhibited flames such as burned gas temperature, burning velocity, stable product species concentrations and flames thickness and detailed flame structure measurements. Both these approaches yield information important to understand the chemistry of inhibition, and to validate or develop kinetic models. The early stages of this project measure global properties of inhibited flames and later detailed flame structure measurements will be performed.

The results of this research are expected to increase an understanding of the mechanisms of existing fire suppressants which will aid U.S. industry develop effective and environmentally benign agents.

\section{Recent Results}

Notarianni, K.A. and Jason, N., editors, Water Mist Fire Suppression Workshop, March 1-2, 1993 Proceedings, NISTIR 5207, National Institute of Standards and Technology, Gaithersburg, MD, June 1993.

\section{Grants}

"Chemical Inhibition of Methane-Air Diffusion

Flames," K. Seshadri, University of California, San Diego.

"Basic Research on Fire Suppression," A. Atreya, University of Michigan. 


\section{Optimization of Aircraft Fire Suppression System Discharge}

Principal Investigator:

Jiann C. Yang

Fire Science Division

301-975-6662

\section{Sponsor:}

Department of the Air Force

Wright Laboratory

Objective

To ensure that the four recommended agents for full-scale testing by the Air Force are delivered to the fire in an efficient manner so that an unbiased evaluation of system effectiveness can be made and to provide generic scientific data to fire suppression system suppliers and airframe manufacturers in support of their efforts to design protective systems for existing and future aircraft.

\section{Problem}

None of the gaseous agents being screened for the Air Force has exhibited the chemical activity of halon 1301. The mass concentrations of many of the agents needed to suppress the laboratory flames are closely clustered. The thermodynamics properties of these agents, however, vary significantly.

\section{Approach}

During FY 1994, BFRL will determine the practical limits to the design of system hardware which constrain what the Air Force can do in the full-scale evaluation. Temperature-pressure data will be collected on the recommended fluids, pure and in mixtures with nitrogen, to enable the development of appropriate equations of state from -60 to 150 ${ }^{\circ} \mathrm{C}$, and for pressures up to $40 \mathrm{MPa}$. It will be determined how the initial conditions in the storage bottle and the design of the release mechanism and exit orifice can be modified to enhance the discharge rate of the recommended fluid agents directly into an unconfined space. The performance of the recommended fluid agents flowing through various piping geometries and components in transient operation will be determined.

The expected benefits of this work will provide design information for in-flight fire suppression bottles for recommended agents, provide guidelines to optimize the discharge rates of recommended agents into dry bays, and provide engineering correlations for the transient two-phase pipe flow of the agents.

Recent Results

New project. 


\section{Real-Time Suppressant Concentration Measurement}

Principal Investigator:

William M. Pitts

Fire Science Division

301-975-6486

\author{
Sponsor: \\ Department of the Air Force \\ Wright Laboratory \\ Survivability Enhancement Branch \\ Department of the Navy \\ Naval Air Systems Command \\ Department of the Army \\ Tank Automotive Command \\ Federal Aviation Administration \\ FAA Technical Center
}

\section{Objective}

To evaluate possible methods for real-time measurement of concentrations of alternative fire fighting agents for dry bay and nacelle applications.

\section{Problem}

The U.S. Air Force is coordinating the search for alternative fire fighting agents for dry bay and nacelle fires on aircraft. They have narrowed their search to four candidates--HFC-227, HFC-125, FC218 , and $\mathrm{CF}_{3} \mathrm{l}$. A method is required for measuring real-time concentrations of these agents during fullscale nacelle and dry bay tests. Existing measurements techniques do not have the required temporal resolution.

\section{Approach}

During FY 1994, BFRL will perform a 2 part approach:

1. Perform a literature search of possible methods for concentration measurement and those that should be tested as part of this research, that have applicability to meeting the needs of the Air Force. 2. Test and characterize two instruments--a differential infrared broad band absorption device developed under an Air Force SBIR and an aspirated hotfilm concentration probe used for qualitative flow characterization during last year's effort on agent screening. Provision is included for testing one of the instruments in Wright Patterson's full-scale test facilities if one of the techniques appears to be viable.

Recent Results

Nev project. 
Comparison of Fire Sprinkler Piping Materials, Steel, Copper, Chlorinated Polyvinyl Chloride, and Polybutylene, in Residential and Light Hazard Installations

Principal Investigator:

Kathy A. Notarianni

Fire Science Division

301-975-6883

\section{Sponsor:}

Federal Emergency Management Agency

U.S. Fire Administration

\section{Objective}

To develop information to help decision makers select sprinkler pipe for residential and light hazard installations.

\section{Problem}

Materials available for use in piping systems for residential sprinklers are grouped into two categories - metals and plastics. The metals include standard-weight steel, lightweight steel, and copper tubing. The plastics include chlorinated polyvinylchloride (CPVC) and polybutylene (PB). While these materials are capable of transporting water to the sprinklers, each has advantages and disadvantages when used in a fire sprinkler system depending on the particular application. The enduser requires information to select piping material.

\section{Approach}

During FY 1994, BFRL will compare and evaluate the following key variables:

- corrosive conditions

- materials degradation during a fire

- maintenance

- economics

- occupancy classification limitations

- system modification limitations

- materials limitations with types of systems

- $\quad$ system design (hydraulic calculations)

- hanger and bracing considerations

A matrix of key variables affecting sprinkler pipe selection will be produced.
Recent Results

New project. 


\section{Smoke Movement and Smoke Layer Development in High Bay Areas}

\section{Principal Investigator:}

Kathy A. Notarianni

Fire Science Division

301-975-6883

\section{Sponsor:}

National Aeronautics and Space Administration Goddard Space Flight Center

\section{Objective}

To develop data on smoke movement and layer development in high bay areas to permit NASA to make decisions about fire detection and suppression in their high bay spaces.

\section{Problem}

There is a special need to address fire protection issues for high ceiling height large spaces. NASA has numerous high bay spaces that are used to perform a variety of functions. Examples include those used for clean rooms, shuttle simulators, assembly/storage, vacuum and vibration chambers, vehicle assembly, and/or testing facilities with payloads. These spaces represent some of the most difficult fire protection challenges in that detection of a fire in a large space may be delayed due to the distance smoke and products of combustion must travel to reach the detector, the large amount of ambient air for smoke dilution, the high dollar value of these spaces and the low damage threshold of a clean room. Some of these spaces also involve forced airflow.

\section{Approach}

During FY 1994, BFRL will conduct a survey of NASA high bay spaces to quantify materials and fire sizes. Existing detection and suppression systems will be documented as well as forced air flows. A matrix of NASA spaces will be produced. 3D Modeling will be used to determine the appropriateness of available detection and suppression systems to each type of NASA space. A final applications matrix will be produced so that NASA can make decisions on type and location of fire detection and suppression equipment for its high bay spaces.

\section{Recent Results}

Notarianni, K.A. and Davis, W.D., The Use of Computer Models to Predict Temperature and Smoke Movement in High Bay Spaces, NISTIR 5304, National Institute of Standards and Technology, Gaithersburg, MD, 1993. 


NGT 